\title{
NON-VOLATILE LIQUID-FILM-EMBEDDED MICROFLUIDIC VALVE FOR MICROSCOPIC EVAPORATION CONTROL AND CONTACTLESS BIO-FLUID DELIVERY APPLICATIONS
}

\author{
A Dissertation \\ Presented to \\ The Faculty of the Graduate School \\ University of Missouri-Columbia \\ In Partial Fulfillment \\ Of the Requirements for the Degree \\ Doctor of Philosophy \\ By \\ RIBERET ALMEIDA
}

Dr. Jae Wan Kwon, Dissertation Advisor

DECEMBER 2013 
The undersigned, appointed by the dean of the Graduate School, have examined the dissertation entitled

\section{NON-VOLATILE LIQUID-FILM-EMBEDDED MICROFLUIDIC VALVE FOR MICROSCOPIC EVAPORATION CONTROL AND CONTACTLESS BIO- FLUID DELIVERY APPLICATIONS}

presented by Riberet Almeida,

a candidate for the degree of doctor of philosophy of Electrical Engineering, and hereby certify that, in their opinion, it is worthy of acceptance.

Dr. Jae Wan Kwon, Dissertation Advisor

Electrical and Computer Engineering

Dr. Satish Nair

Electrical and Computer Engineering

Dr. Naz Islam

Electrical and Computer Engineering

Dr. Hao Li

Mechanical and Aerospace Engineering 


\section{ACKNOWLEDGEMENTS}

I would like to thank my advisor Dr. Jae W. Kwon, whose guidance, wisdom and knowledge have helped me tremendously in completing my PhD. Dr. Kwon's insight into the field of study has helped me discover newer areas of research not discovered previously.

I would also like to thank my committee members, Dr. Nair, Dr. Li and Dr. Islam for their invaluable time and effort in being a part of my committee.

I would like to thank my colleagues Tongtawee Wacharasindhu (now with Intel, Portland), Quang, Jian, Chris, Brad and Dan for all their help. I would also like to thank Dr. Kovaleski and some of his group members - Pete, Emily, Jim and Brady, who assisted me once in a while with experimental tools.

I would also like to thank Dr. Holger F. Bohn from the Botanischer Garten der Universität Freiburg and Dr. Walter Federle from the University of Cambridge, who have forwarded their SEM images of the Nepenthes pitcher plant for our study and subsequent publication of slippery surfaces.

My gratitude also extends to members of the Electron microscopy core facility - Randy, Juliana, Wen and Lou Ross, who have helped me in the high resolution imaging of micro/nanostructured surfaces.

I would also like to thank my parents and siblings for their invaluable help both financially and morally during this time.

Lastly I would like to thank my wife Theresa for her relentless patience and help during this time. 


\section{TABLE OF CONTENTS}

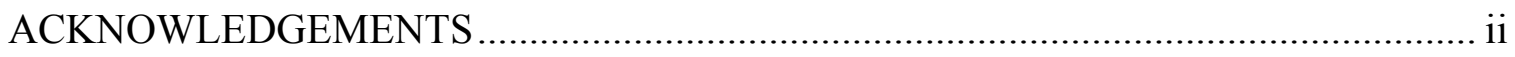

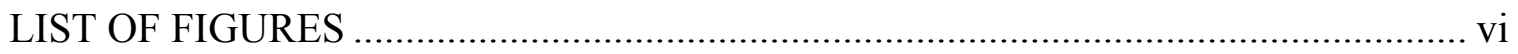

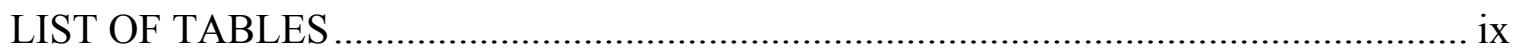

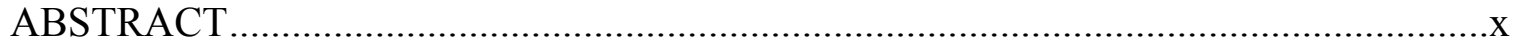

Chapter

1. INTRODUCTION

1.1.Microfluidics - Fluid handling at the microscale............................................

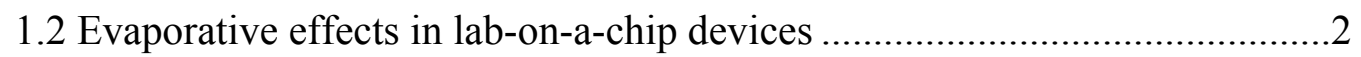

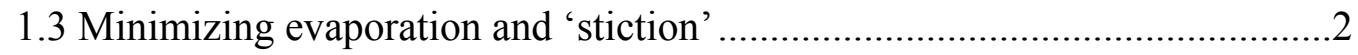

1.4 Anti-clogging mechanisms in inkjet printers .................................................

1.5 Bio-inspired tools for efficient microfluidic valve driving ............................

1.6 Overview of chapters ……………………………..............................

2. NONVOLATILE LIQUID-FILM-EMBEDDED MICROFLUIDIC VALVE FOR MICROSCOPIC EVAPORATION CONTROL

2.1 Design and theory - Bio-inspired design ....................................................10

2.2 Evaporation rate control at the liquid-air interface ………..........................13

2.3 Driving of liquid-film acting as a valve-core ……........................................15

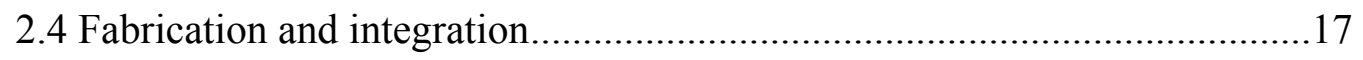

2.5 Study of liquid-core materials for various bio-ink materials ........................19

2.6 Choice of dielectric layer …………………………...............................21

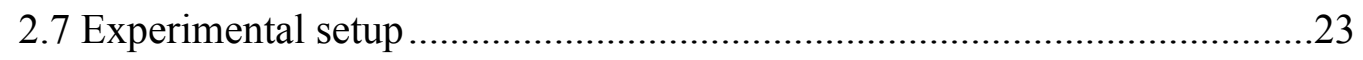

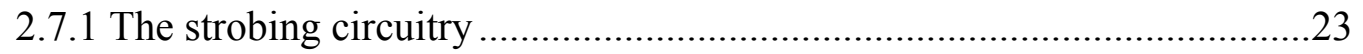

2.7.2 High speed imaging technique for the ejector...........................................25 
2.8.1 Testing of the liquid-core microfluidic valve......................................26

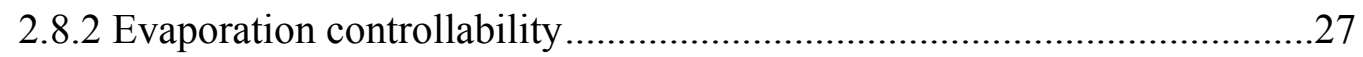

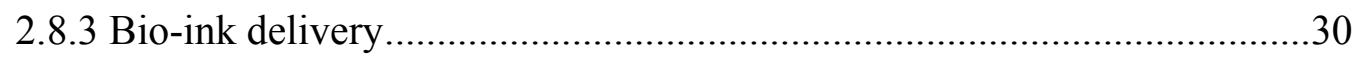

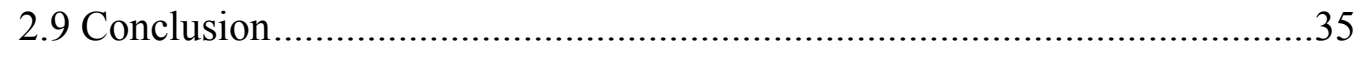

\section{LOW SURFACE TENSION LIQUID CONFINEMENT USING OIL-} REPELLENT SURFACES

3.1 Oil-repellent surfaces and virtual walls for guiding low surface tension

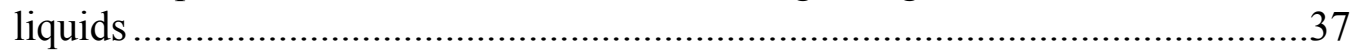

3.2 Fabrication details for oil-repellent surfaces .........................................43

3.3 Study of virtual wall driven capillary flow .........................................45

3.4 Study of defect initiated contact line pinning ......................................48

3.5 Characterization of oil repellent surfaces using contact angle

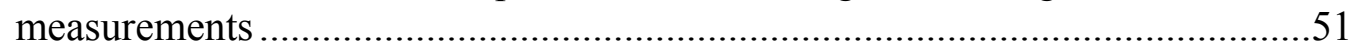

3.6 Guiding of the liquid-core microfluidic valve on ejector surface using oilrepellent surfaces.

3.7 Fabrication and working mechanism of guiding features on ejector surface

4. LOW VOLTAGE DRIVING OF MICROFLUIDIC VALVE AND DEVELOPMENT OF SLIPPERY SURFACES

4.1 Development of hydrophobic / oleophobic surfaces for reduction in microfluidic valve driving voltages.

4.2 Slippery surfaces for open channel microfluidic valve actuation 61

4.3 Motivation for developing slippery surfaces........................................62

4.4 Learning from nature: the Nepenthes pitcher plant.................................64

4.5 Bio-mimicked surfaces with imbibed functionality ..............................65

4.6 Conditions needed to develop slippery surfaces ..................................69

4.7 Mechanisms governing slippery surfaces ..........................................70 
4.8 Characterization of optical properties of slippery surfaces .......................81

4.9 Fabrication details and methods for slippery surfaces .............................84

5. LONG TERM RELIABLE BIO-FLUID PRINTING WITHOUT NOZZLE FAILURES

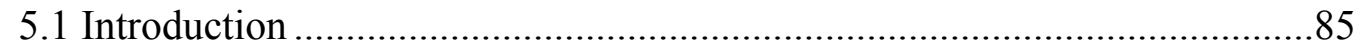

5.2 Inkjet printing for contactless bio-fluid delivery applications ...................85

5.3 Sliding Liquid drop (SLID) technology for use in bio-printers ...................88

5.3.1 Currently adopted techniques for clog-free ejection ............................88

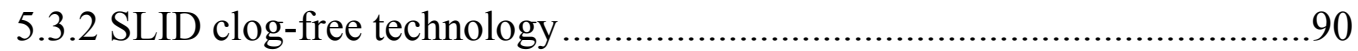

5.4 Fabrication details of ejector integrated with the SLID shutter .................93

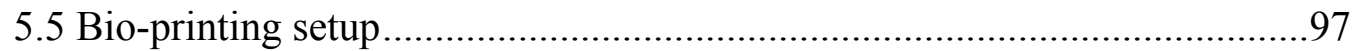

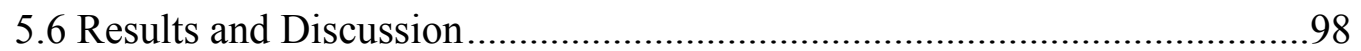

5.6.1 Testing of the SLID shutter mechanism................................................98

5.6.2 Printing of bio-fluids including proteins and hydrogels........................101

6. FUTURE RESEARCH AND CONCLUSION .............................................. 110

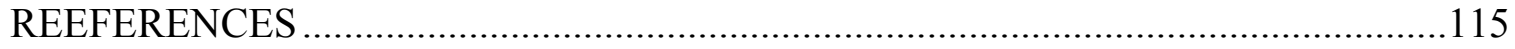

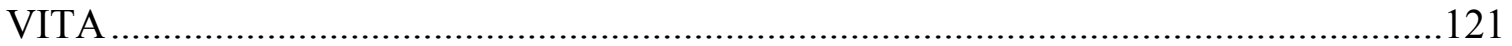




\section{LIST OF FIGURES}

Figure

1. Illustration of three layers of the tear film in human eye

2. Schematic illustration of the device with the microfluidic valve in

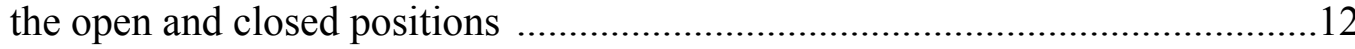

3. Cross-section view of liquid-film-based microfluidic valve ..............................13

4. Fabrication steps for ejector with microfluidic valve ......................................18

5. Strobing circuitry and typical values of strobing circuit ....................................22

6. Images of ejected ink droplets using the new strobing circuitry ............................24

7. Schematic of the test setup with short pulse stroboscopic illumination to image

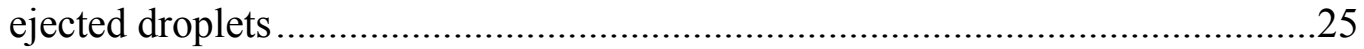

8. Images of a LiFE microfluidic valve using silicone oil in 'open' position and

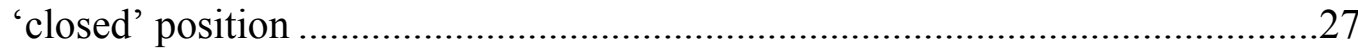

9. Images of growing air pocket size in ejecor when valve is 'open' and corresponding graph

10. Images of ejected droplets through cover orifice while the $\mathrm{LiFE}$ valve is left open.

11. Ejection images of protein streptavidin without the initial top coverslip.

12. Ejection testing with the use of viscosity modifiers for improved ejection

13. Ejected hydrogel with Rhodamine $6 \mathrm{G} \&$ Acridin orange imaged using

Fluorescence microscope

14. Illustration of conventional superoleophobic and oil-repellent surfaces

15. Images of a silicone oil droplet sliding towards a superoleophobic region

16. PDMS droplet at the edge of an oil-repellant surface.

17. Oil droplets pneumatically actuated towards textured oil-repellant region

18. Fabrication steps for developing virtual wall microfluidic device. 
19. Illustration of important parameters for the virtual walls

20. Schematic of cross section of liquid stream between parallel plates

21. Illustration of the contact angle due to TCL on a homogenous surface and at a defect

22. SEM image of a PDMS droplet at the boundary between an oleophilic and oil repellent-area

23. Graph of contact angles vs surface tension of various low surface tension liquids.

24. Optical microscope image of underlying electrodes confined and elongated silicone oil droplet.

25. Fabrication details of dielectrophoretic device for manipulating confined microfluidic valve.

26. Droplet actuated across the desired path using liquid

Dielectrophoresis (L-DEP).....

27. SEM images of cured photoresist microstructures and electroless Nickel microstructures.

28. Bio-inspired electrode design and surface morphology of Nepenthes

Bicalcarata pitcher plant

29. Oil drainage from grooved and un-grooved flat region on the sample

30. SEM images of the nanoparticle/Cytop coating on the electrodes and contact angles

31. SEM images of plasma induced nano roughness within grooves and contact angles

32. SEM characterization of slippery surfaces - Cross section and zoomin

33. Differential Laplace pressure initiated droplet movement on slippery surface.

34. Cross-sectional schematic view of slippery surface

35. Reversible change in contact angle and droplet actuation on slippery surface.

36. Actuation of liquid droplets on slippery surfaces .80 
37. Slippery surface characterization using contact angle measurements.

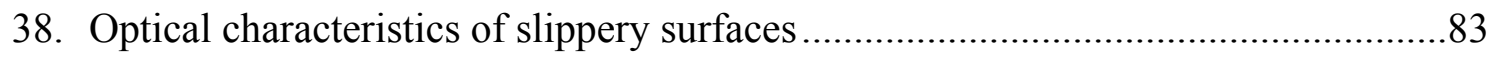

39. Schematic of the normally-closed SLID shutter on the ejector plate......................93

40. Image depicting the various steps of SLID shutter fabrication

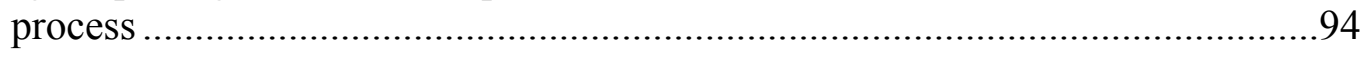

41. Images of different areas on the fabricated device - the SLID shutter ..................96

42. SEM images of modified / defect-induced area ...............................................98

43. Top view of the SLID shutter in the closed and open state...................................99

44. SLID shutter actuated from the 'closed' state to the 'open' state ..........................101

45. View of ejected droplets with SLID shutter in the open position .........................101

46. Fluorescence microscopy images of hydrogels printed on bare glass

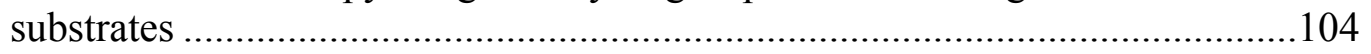

47. Optical microscopic images of Adenosine ejected on bare glass

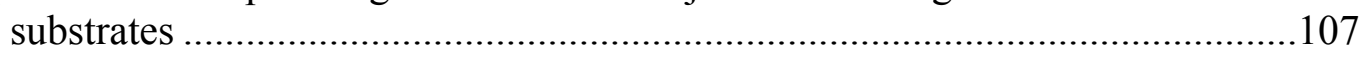

48. Fluorescence microscopic images of Adenosine ejected on patterned

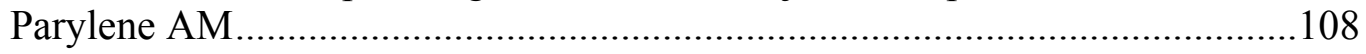




\section{LIST OF TABLES}

Table

1. A comparison of the different liquids to be used for the liquid film along with the actuation voltage, dielectric constant, solubility in water, surface tension, vapor pressure and toxicity.....

2. The different dielectric materials that were used on the ejector surface along with the dielectric constant, breakdown voltage and film thickness

3. Evaporation speed data when the LiFE microfluidic valve is opened or closed......30

4. All the proteins that were tested in the ejector with the microfluidic valve.

5. Actuation voltages with and without the use of micro textured guiding of various low surface tension liquids.

6. Protein solutions tested in the microfluidic ejector equipped with the SLID shutter

7. Evaporation speed data for various hydrogel solutions when the SLID shutter is opened or closed 


\begin{abstract}
Quick evaporation speed of microfluids can cause many unexpected problems and failures in various microfluidic devices and systems. In this dissertation, a new evaporation speed controlling method is demonstrated using a thin liquid-film based microfluidic valve. Microfluidic droplet ejectors were designed, fabricated and integrated with the liquid-film based microfluidic valve. The thin liquid film with non-volatility and immiscibility exhibited excellent microfluidic valve functionality without any stiction problem between valve components, and provided a very effective evaporation protection barrier for the microfluids in the device. Successful evaporation control by the liquid-film-embedded (LiFE) microfluidic valve has been demonstrated. In addition, guided actuation of the microfluidic valve along predefined paths was successfully achieved using newly developed oil-repellent surfaces, which were later used for developing 'virtual walls' for confining low surface tension liquids within predefined areas. Moreover, bio-inspired slippery surfaces for aiding the microfluidic valve along the ejector surface have also been developed. These slippery surfaces were evaluated for their effectiveness in reducing microfluidic valve driving voltages. Finally, a sliding liquid drop (SLID) shutter technique has been developed for a normally-closed functionality with aid from nanostructures. The SLID shutter resolves many issues found in the previous LiFE microfluidic valve. Smooth and successful printing results of highly volatile bio-fluids have been demonstrated using the SLID shutter technique. I envision that these demonstrated techniques and developed tools have immense potential in various microfluidic applications.
\end{abstract}




\section{Chapter 1 \\ INTRODUCTION}

\subsection{Microfluidics - Fluid handling at the microscale}

Microfluidics is an interdisciplinary field which deals with small amounts of liquids (from $10^{-9}$ to $10^{-18}$ liters) in micron-sized channels and containers. In this size range, surface area processes become much more salient than those of volume. For example, heat and mass transfer become much more prominent due to the large surface area to volume ratio $[2,3]$. Scaling down of fluidic processes to the microscale offers many significant advantages associated with size reduction and integration. One of the biggest advantages in implementing miniaturized components and processes is the very small volumes of fluids, thereby reducing reagent consumption and the cost of processes. Furthermore, diagnostic results as well as reagent products can be obtained in a much shorter time which further reduces the cost because of the reduced time scale. Also the level of sensitivity is increased due to selectivity of the process and the higher precision.

However, a lot of the undesirable features become prominent in the microscale. For example, quick evaporation speeds of fluids in the microscale is a critical problem and can cause many unexpected results and failures in microfluidic devices. In general, vaporization of microfluids at the microfluidic ports with open liquid-air interfaces can easily leave evaporation residues, which often cause port failures. 


\subsection{Evaporative effects in lab-on-a-chip devices}

With the broad utilization of various Lab-on-a-chip (LOC) devices, even very small liquid volumes can be readily and easily analyzed as compared to many traditional lab-based analytical tools. Multi-process capability in a tiny device is very attractive, and various small lab-on-a-chip devices find applications in the life sciences, chemistry and the other disciplines of sciences ranging from mass spectrometry to tissue building micro droplet dispensers [4-8]. However, it is still very difficult to use microfluidic devices for many labon-a-chip applications due to the extremely rapid evaporation of microfluids through tiny open ports with liquid-air interfaces. For example, ten nanoliters of water evaporates within thirty seconds under a standard laboratory environment whereas ten picoliters of water evaporates in less than a second [9]. In general, vaporization of microfluids at the microfluidic ports can easily cause significant volume loss, concentration, osmolarity and even viscosity change of the microfluids [10]. Thus, inevitably quick evaporation has been a very critical problem for many microfluidic systems having open liquid-air interfaces.

\subsection{Minimizing evaporation and 'stiction'}

In macroscale, the most straightforward and common way to avoid the rapid evaporation is to completely cover or block the liquid-air interface by using a valve so that its direct air exposure can be prevented. However, in microscale, covering/uncovering the liquid-air interface with generic microfluidic valve mechanisms utilizing solid mechanical parts without stiction related issues is a big challenge. Several approaches for minimizing the quick evaporation problems in various microfluidic systems by employing humidified environments [9, 10], sacrificial liquids [11], and optimized geometries [12] were 
attempted by several researchers. But problems associated with quick evaporation speeds had not been previously completely resolved. Most of the previous techniques relied on retardation of evaporation rates by altering the surrounding environment. In some other cases, unwanted condensation by the presence of temperature gradients may cause even worse problems [13]. Furthermore, quantitative analysis for assessing reduction in evaporation rates has been very limited.

In retrospection to previous efforts, an easier method would be to simply use a microfluidic valve to cover/uncover the fluidic port whenever needed. In this way, the evaporation rate can be entirely curbed while the valve is in the closed state. However, tiny valve structures are often vulnerable to stiction, which is a well-known major problem in the microscale. Stiction is caused by adhesion or interfacial bonding and becomes even more problematic when the two solid surfaces are brought into contact with a liquid. Thus, solid mechanical components in microfluidic devices can be simply immobilized due to the strong adhesive forces comprised of the meniscus and viscous forces. When stiction occurs, the restoring force of the component is not enough to release it from the liquid. Stiction can be reduced by various methods and one of the commonly used methods is reducing the actual contact surface area with tiny bumps [14]. Such roughened surfaces, however, cannot provide an ideal condition of leak-tight sealing required for a 'zero evaporation' condition. Thus, it seems not trivial to control microscopic evaporation at the liquid-air interface in a microfluidic port. 


\subsection{Anti-clogging mechanisms in inkjet printers}

Desktop inkjet printers too have been plagued with the problem of quick evaporation at the printhead nozzles leading to clogging. Since pigment-based inks are commonly being used nowadays and have a higher solid content than previous dye-based inks, the problem is even more critical. However, pigment-based inks are preferred as they produce deeper contrasts with colors appearing more vivid and vibrant in printed images. Nevertheless, both of these ink types - pigment and dye based - are designed for fast drying. However, the tiny nozzles and quickly drying ink increase the vulnerability of ejectors to clogging due to the solids within these inks in addition to dried ink residues at the nozzles [15].

Desktop inkjet printers use different techniques to prevent clogging in inkjet printhead nozzles from occurring. Some inkjet printheads are equipped with a wiper cleaning mechanism to wipe the inkjet printheads [15]. The wiper removes ink residue and other contaminants from the printhead surface through a wiping motion. In addition, a service station attached to the printer is used for preventative maintenance. When not in use, the printhead is safely stored at the service station which caps and seals the printhead nozzles from contaminants and drying. Furthermore, during operation, clogs in the printhead are periodically cleared by firing a number of ink droplets through each of the nozzles in a process known as 'spitting' [15]. Immediately after spitting, uncapping or during printing, an elastomeric wiper at the service station wipes the printhead surface for further cleaning. During this time, any ink residue, particulates or other contaminants on the printhead are cleared off. Dye-based inks are simpler for cleaning purposes as a simple wiping motion accomplished by the elastomeric wiper is sufficient. However, additional mechanisms are needed when working with pigment based inks. These ink residues are sticky by nature and 
adhere to the wiper. Most often an additional scrapper is needed to clean the wiper and rid the sticky pigment residue off its surface [15]. This can sometimes cause additional problems due to mechanical wear and tear to the elastomeric wiper.

Therefore, many problematic phenomena described above have been studied in depth and the possibility of exploiting immiscibility between fluids for evaporation control has been thoroughly explored. The spatial controllability of immiscible liquids using electrical actuation schemes has also been explored. An immiscible liquid film, has been engineered and demonstrated for evaporation speed controlling means using a thin liquid film based microfluidic valve in a microfluidic droplet ejector. The non-volatile and immiscible thin liquid film exhibits microfluidic valve functionality without any stiction problem between valve components and provides a very effective evaporation protection barrier for the microfluids in the device. Successful evaporation control by the liquid-film-embedded (LiFE) microfluidic valve has been accomplished.

\subsection{Bio-inspired tools for efficient microfluidic valve driving}

Learning from nature has been another scientific discipline that researchers have been actively pursuing. Biomimetics is defined as the study of the structure and function of biological systems as models for the design and engineering of materials and machines. Millions of years before researchers engineered bio-inspired materials, biological systems had evolved with features having dimensions down to the nanoscale while exhibiting remarkable functionalities. Today, scientists have been mimicking biological systems in the quest for developing newer technologies [16]. Specifically, scientists have been trying to biomimic natural non-wetting surfaces for controlling the flow of liquids in microfluidic 
devices as well as for developing microfluidic components. Mimicking the lotus leaf was one of the first attempts in developing artificial textured surfaces inspired from nature [1719]. It was from this plant that researchers understood the mechanisms that govern the nonwetting properties of natural surfaces. It is from the lotus plant that scientists learned how superhydrophobic surfaces could be synthesized. By understanding the various parameters governing the surface features of naturally occurring superhydrophobic surfaces, scientists were able to develop artificial superoleophobic surfaces. These surfaces are far superior to their hydrophobic counterparts and can be used for repelling aqueous as well as nonaqueous oily liquids. Superoleophobic surfaces were initially explored for use on the microfluidic ejector. During this time, we discovered a new type of surface that was highly repellent to liquid films moving in the lateral direction. We also realized that these surfaces - labelled as oil-repellent surfaces - could be used for guiding non-aqueous low surface tension liquids in microfluidic channels. This is the first time that such a liquid transport phenomenon has been demonstrated for low surface tension liquids through the use of 'virtual walls'. The design of certain features of these oil-repellent surfaces can be traced back to superoleophobic surfaces which can further be traced back to the superhydrophobic leaves of the lotus plant. These surfaces have immense potential for use in lab-on-chip (LOC) devices and for other microfluidic applications where non-aqueous oily liquids are utilized.

Another area in microfluidics that has evoked widespread interest especially of those in the lab-on-chip (LOC) community has been the dynamic control of surface wettability for the purpose of guiding liquid droplets across surfaces. Different actuation schemes implementing electric fields for varied applications have been exhaustively explored for 
this purpose in the last decade. This area of research continues to develop and grow and is one of the major categories in microfluidics. On the other hand, the development of nonwetting surfaces - repellent to aqueous and / or non-aqueous liquids, is another area of microfluidics that has continued to develop over the years. Robust omniphobic surfaces which comprise micro and nano-engineered surfaces fabricated using bottom-up, top-down and self-assembly belong to this category. These surfaces which are repellent to aqueous / non-aqueous liquids, biological fluids as well as oily liquids - liquids spanning a large gamut of surface tensions - have also garnered widespread interest in the last few years. While the dynamic control of liquids as well as the development of non-wetting / selfcleaning surfaces can be looked upon as mainstream microfluidic sub-categories, there has been almost no overlap between these two categories. In this regard, the design of surfaces that can repel as well as manipulate liquid droplets on their surface has not been explored much and continues to pose a great challenge. Despite great advances in synthesizing surfaces that are ultra-repellent to liquids, there are still challenges that need to be addressed for the purpose of droplet mobility. To elucidate further, most of these nonwetting surfaces rely on air cushioning to remain stable which can destabilize under the influence of electric fields. Recently, efforts have been focused on replacing the 'air cushioning' with lubricant liquids thus improving stability. While exploring superoleophobic surfaces on the ejector in the quest for lowering the driving voltage, we engineered a liquid infused non-wetting surface with the addition of a mobility function. These engineered surfaces have been shown to repel naturally as well as electrically in some cases, different liquids such as hydrocarbons, crude oil and biological fluids such as blood, milk, water etc. These surfaces have immense potential for surface microfluidic 
systems where the dynamic transport of liquid droplets across surfaces using electric fields is a requirement while preserving the stability of their non-wetting characteristics.

\subsection{Overview of chapters}

A droplet ejector integrated with a microfluidic liquid valve is used to demonstrate how evaporation occurring at its nozzle liquid-air interface can be completely blocked. Chapter 2 describes in detail the fabrication, the working mechanism and the implementation of the liquid-core microfluidic valve. This is followed by experimental results substantiating the effective cessation of evaporation taking place at the nozzle. The spatial controllability of the microfluidic valve which is demonstrated using electro actuation schemes is also described in this chapter. Chapter 3 describes the design of newly developed oil-repellent surfaces which are used for guiding liquids such as the microfluidic valve liquid along surfaces. This chapter also describes how microfluids such as the microfluidic valve can be manipulated electrically while being confined along predefined paths on surfaces. The development of micromachined features that are compatible with CMOS related processes and which are used to make these surfaces oleophobic is also described in this chapter. In Chapter 4, the development of omniphobic surfaces that are used to repel aqueous as well as non-aqueous liquids has been described. In the quest for reducing the microfluidic valve driving voltages for droplet ejector applications, these surfaces were conceptualized, synthesized and extensively explored. During this time, a new class of bio-inspired surfaces were developed that were labeled as 'slippery surfaces'. In this chapter, these surfaces were thoroughly explored to understand their slippery mechanism. Chapter 5 introduces a new 'Sliding liquid drop' (SLID) mechanism which is equipped to a microfluidic ejector nozzle for controlling the evaporation through the nozzle. Furthermore, nanostructures were 
exploited for imparting a normally-closed characteristic to the liquid shutter. This chapter describes in detail the use of the liquid shutter which acts as a valve mechanism and enables the printing of highly volatile bio-fluids. Finally, chapter 6 concludes with a future plan in each of the different areas explored and presents a brief summary of all the chapters. 


\section{Chapter 2}

\section{NONVOLATILE LIQUID-FILM-EMBEDDED MICROFLUIDIC VALVE FOR MICROSCOPIC EVAPORATION CONTROL}

\subsection{Design and theory - Bio-inspired design}

Nature has evolved biological features in living organisms exhibiting enhanced performance using commonly found materials. The functionality of these features range from the macroscale to the nanoscale. Understanding the functions and processes developed by biological features found in nature can guide us to imitate and produce nanomaterials, nano-devices and influence the design of micro-electro-mechanical systems (MEMS). Biologically inspired design or adaptation or derivation from nature is referred to as 'biomimetics'. It means mimicking biology or nature. Biomimetics is a highly interdisciplinary field of study. It involves the understanding of biological functions, structures and principles of various objects found in nature by biologists, physicists, chemists and material scientists, and the design and fabrication of various materials and devices of commercial interest by engineers, material scientists, chemists and others.

In trying to solve the fundamental problem of clogging in inkjet printers we have turned to nature. And in doing so we were able to solve the problem of drying nozzles. We have introduced a newly invented method, which can overcome all the problems described above and provide excellent microscopic evaporation controllability. Such a technique has been originally inspired by nature; in human eyes, an oily liquid layer protects tear evaporation and avoids dryness of the eye balls very effectively. The tear film (precorneal film) has three distinct layers as shown in Figure 1. The outermost oily lipid layer (secreted by the meibomian glands) coats the underlying aqueous layer and provides a hydrophobic 
barrier, retarding evaporation of tears. Thus, a liquid can be an effective material to control the evaporation of another liquid. Likewise, we have utilized a thin protective liquid film on top of another liquid.

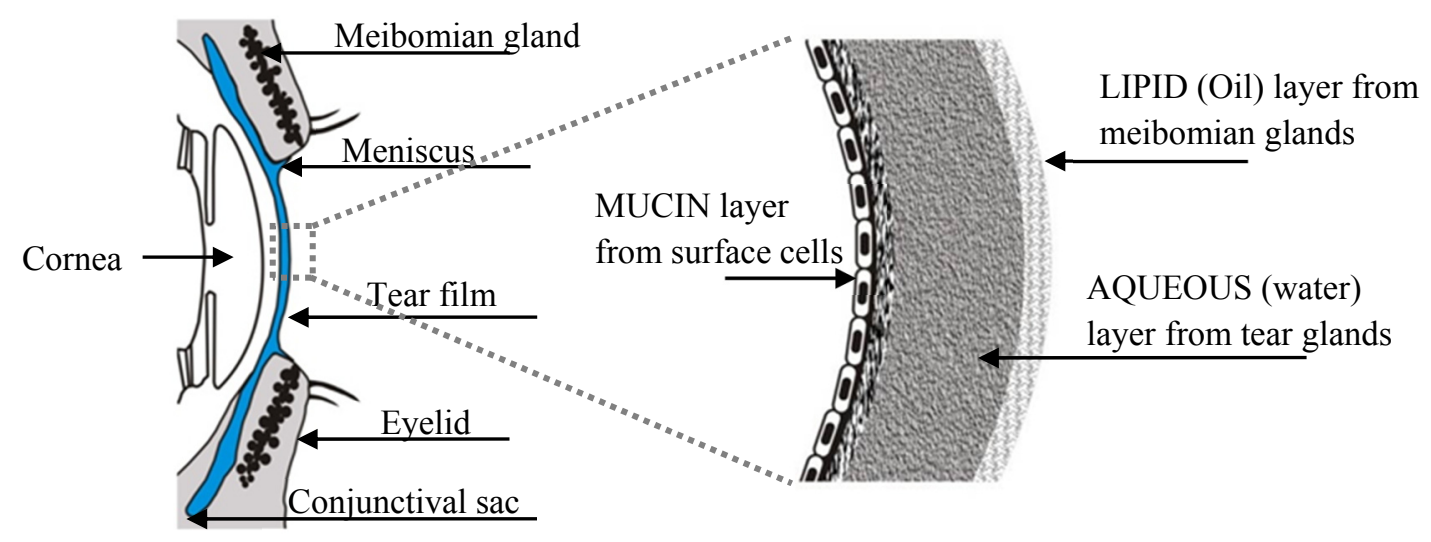

Figure 1. Illustration of three layers of the tear film in human eye. A thin oily layer prevents quick evaporation of the aqueous layer very effectively.

Eyelids help keep the thin lipid (oily) layer effectively over the eye ball by blinking, which is not an easy or efficient way to handle microfluidics. In our design, we have added simple electrical microfluidic motion controllability on the protective liquid layer. Interestingly, liquids have numerous interesting and unique properties, which are very helpful for our application, as follows; 1) some liquids do not stick on hydrophobic surfaces but move very easily; 2) some liquids evaporate very slowly and almost negligibly; and 3) some liquids cannot be mixed with other liquids and are thus completely immiscible. Thus, when a non-volatile and immiscible liquid covers another liquid (working fluid in the system) and forms a thin layer over it, the working fluid can be protected from direct air exposure and its evaporation speed can be significantly reduced. We have studied how such a thin protective liquid film can move for the open/close operation at the microfluidic port to work like a valve. Unlike most microfluidic valves using solid valve-cores (membrane, 
cantilever, etc.), implementing a nonvolatile liquid film as a valve-core is a very novel approach to control the rapid evaporation rate of microfluids.

To prove this concept, we have built a microfluidic system (microfluidic droplet ejector) with an open microfluidic port (nozzle) having an air-exposed interface of the working fluid. And a thin liquid film embedded (LiFE) microfluidic valve was designed to control the quick evaporation problems at the nozzle area. A conceptual design of the device has been illustrated in Figure 2. When the ejector is not in use, the nozzle can be closed to stop the evaporation.
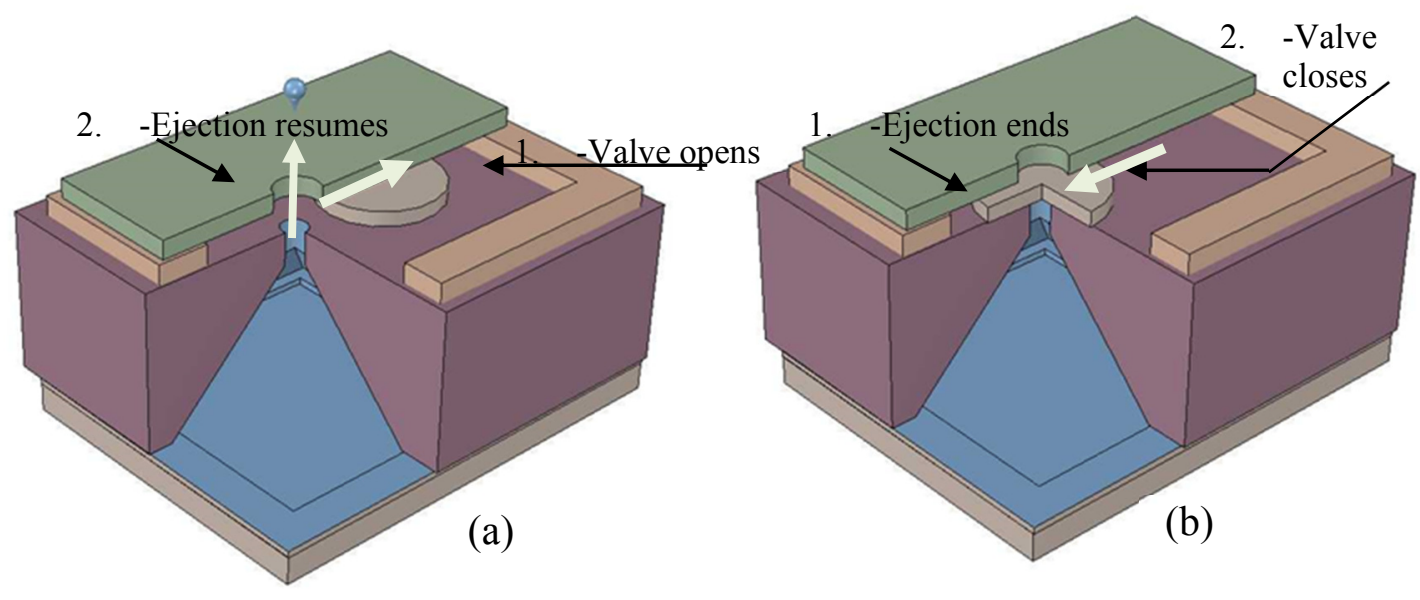

Figure 2. Schematic illustration of the device with the microfluidic valve in the open and closed positions. (a) Ejection is commenced immediately after the valve is opened and continues till the desired amount of fluid has been dispensed. (b) After ejection, the valve is closed to prevent evaporation.

Since the liquid-air interface at the nozzle of the microfluidic ejector is exposed to the ambient air at all times, it is vulnerable to the effects of evaporation. Residue left by quick evaporation of the working fluid in the ejector through the tiny nozzle causes complete blockage/failure of the microscopic fluidic passage [20]. The presence of an evaporation barrier at the interface can prevent the undesirable effects of evaporation. At the time of 
dispensing the working fluid, the barrier can be moved away to open the port, and kept closed for the rest of the time to prevent any evaporative losses as illustrated in Figure 3.

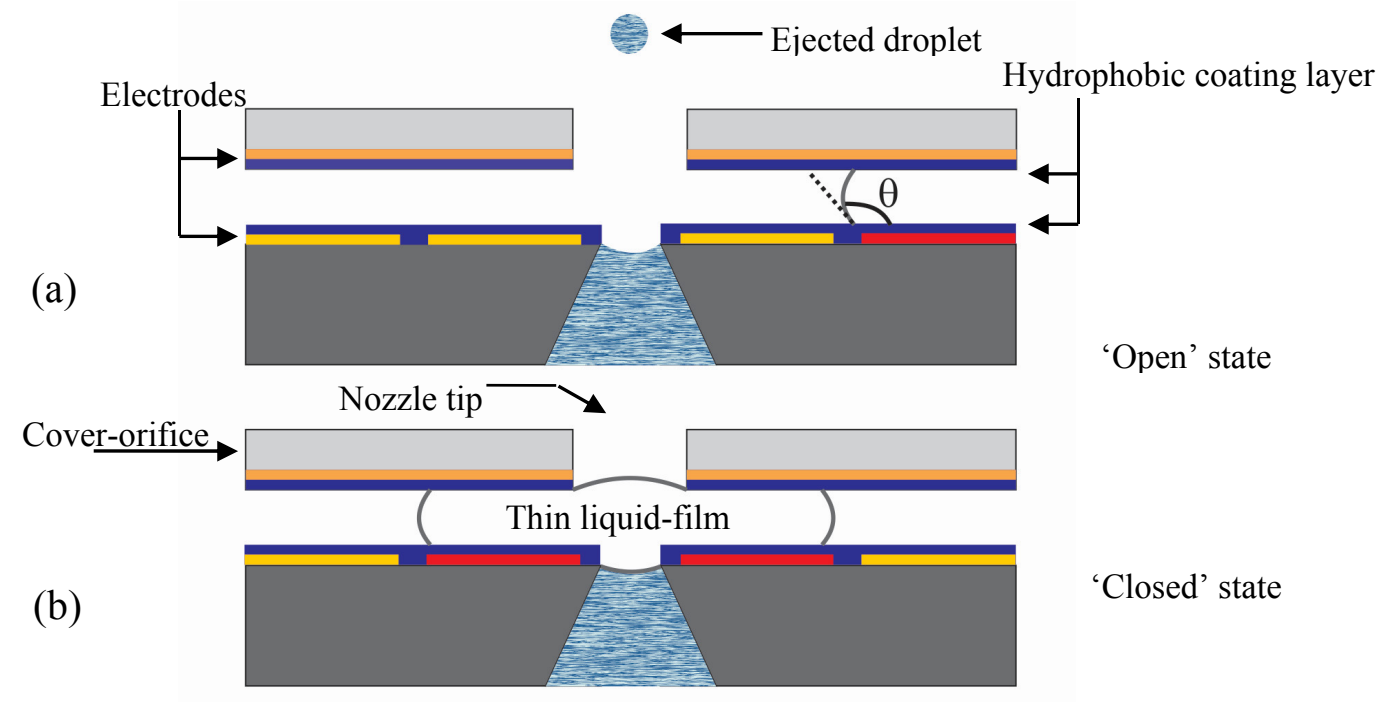

Electrode in ON state

OFF state

Figure 3. Cross-section view of liquid-film-based microfluidic valve. The fluidic valve can be used to open and close the ejector nozzle. (a) Microfluidic valve is in the 'open' state. (b) Microfluidic valve is in the 'closed' state.

\subsection{Evaporation rate control at the liquid-air interface}

Evaporation is a diffusion limited process of water vapor moving away from the air-liquid interface depending on the surrounding conditions such as mass concentration of water vapor or temperature. In the evaporation process liquid molecules interchange rapidly between the surface and the adjacent air so that this air is saturated with vapor. For still air, a concentration gradient develops by diffusion as the air at infinity is not saturated and vapor diffuses outward. Diffusion-limited evaporation can be described by Fick's diffusion equation whereby the diffusive flux is given by $\bar{J}=-D \bar{\nabla} C$ with $D$ being the diffusion 
coefficient and $\nabla C$ being the gradient of concentration. In an ideal state assuming the gas above the meniscus in the nozzle is in a quiescent state, the diffusion process will rapidly attain a steady state and the diffusion equation reduces to the Laplace equation. This then results in a boundary value problem with boundary conditions $C=C_{S}$ (saturation concentration) at the interface and $C=C_{\infty}$ (ambient vapor concentration) far away from the interface. A general expression for the evaporation rate can be given by

$$
E=\frac{D}{\rho_{\text {liquid }}} \int \frac{d C}{d n} d S
$$

where $\rho_{\text {liquid }}$ is the density of the working fluid, $C$ is the mass concentration of the vapor, $n$ is the unit vector normal to the interface and $S$ is the surface of integration.

The evaporation flux can be reduced or completely eliminated if the diffusion of vapor from the liquid-air interface to the outside environment is obstructed by the microfluidic valve. With the microfluidic valve in the closed state, vapor can no longer diffuse outwards as the liquid-air interface no longer exists. This prevents the evaporation rate from having a finite non-zero value.

In order to implement the LiFE microfluidic valve to stop the evaporation at the liquid-air interface, there are important properties required of the liquid film to act as an evaporation barrier. First, complete immiscibility of the liquid film material and the working fluid is highly preferred. Otherwise, the liquid film (valve-core) can contaminate the working fluid over time. In addition, the liquid film material needs to possess a very low to almost negligible vapor pressure so that it can be sustained for a prolonged period of time. Thus, 
by using an immiscible and nonvolatile liquid film on top of the working fluid, it is possible to stop the evaporation flux of the working fluid at the liquid-air interface.

\subsection{Driving of liquid-film acting as a valve-core}

The liquid film can be moved for opening/closing operations of the valve by forces generated by the presence of an electric field. Individually addressable electrodes can determine the next position where the liquid film moves to. The motion of the liquid film can be driven by an electrowetting phenomenon when a conductive liquid film is chosen $[21,22]$. But if the liquid film is dielectric, another driving mechanism is involved. Due to the polarization of the dielectric fluid in response to an external electric field, a dielectrophoresis force is exerted on the liquid film. When an external electric field is applied, the charges bound in the liquid molecules move in response to the electric field. This results in the distortion of the molecular charge density and polarization. Since dipoles are dominant over molecular multipoles, the electric polarization $P$ is defined as the dipole moment per unit volume. The volumetric charge density due to polarization is defined as $\rho_{p}=\nabla \cdot P$. The polarization charge exerts its influence in the form of dipole moments, that is, it acts together with its partner of opposite sign as a charge pair. This charge pair has zero net free charge and has a finite dipole moment due to the displacement of the two polarization charges. Hence, the polarization charge is also referred to as the paired charge. The final expression for this force is written as

$$
f_{D E P}=\rho_{p} E
$$

And the dielectric property of the liquid film is an important parameter for its motion. The driving force exerted on the liquid film can also be written as 


$$
f_{D E P}=\frac{\varepsilon_{o}(\varepsilon-1) W}{2 d} V^{2}
$$

where $V$ is the driving voltage, $W$ is the width of the two parallel plates, $d$ is the spacing between the plates, $\varepsilon_{o}$ and $\varepsilon$ are the absolute and relative permittivity of the liquid. For a given voltage, the driving force would be proportional to the dielectric constant of the liquid film being polarized. And, the higher the driving voltage applied, the stronger the force exerted can be. In addition, the dominant forces opposing the movement of the liquid film are the shear forces at the surfaces of the top and bottom plates and the viscous drag exerted by the surrounding air and the contact-line friction. The differential equation governing the microfluidic valve motion based on a semi-empirical expression is given as [23]

$$
m \frac{d^{2} x}{d t^{2}}=f_{D E P}-\left(\frac{6 u l^{v}}{H}\right)\left(2 \pi r^{2}\right)-\left(\frac{1}{2} C \rho_{f} v^{2}\right)(2 r H)-(\varsigma v)(4 \pi r)
$$

where $m$ is mass of the liquid film, $x$ is the distance travelled, $v$ is its velocity, $u_{l}$ is the viscosity, $C$ is the drag coefficient for a cylinder in cross flow, $\rho_{f}$ is the air density and $\varsigma$ is a proportionality coefficient, which depends on the interaction between the substrate and the liquid at the molecular level. The second term, $\left(\frac{6 u l^{v}}{H}\right)\left(2 \pi r^{2}\right)$ is the opposing viscous force on the liquid film due to the top and bottom plates. The third term, $\left(\frac{1}{2} C \rho_{f} v^{2}\right)(2 r H)$ is the opposing viscous stress, which is estimated by considering the sandwiched droplet as a cylindrical rigid body moving through air. The last term, $(\varsigma v)(4 \pi r)$ is the opposing 
contact line friction force, which is assumed to be proportional to the velocity. Moreover, hydrophobic coatings have been used to maximize the driving force by reducing all the opposing forces.

\subsection{Fabrication and integration}

The underlying mechanism involves a sandwiched liquid droplet (immiscible to the working fluid) moving to cover/uncover the nozzle and acting as the microfluidic valve. The LiFE microfluidic valve sits on top of a microfluidic ejector to control the liquid-air interface. We have fabricated the system and a brief fabrication procedure is shown in Figure 4. In step (a), an ejector nozzle (50 $\mu \mathrm{m}$ in diameter) is formed by an anisotropic silicon etchant $(\mathrm{KOH})$ and cleaned by an ultrasonic agitation. Steps (b) through (e) depict the fabrication of the LiFE microfluidic valve onto the ejector surface. For this, the top surface of the ejector was first coated with a $5 \mu \mathrm{m}$ thick parylene $\mathrm{C}$ layer after being treated with an adhesion promoter (A-174 silane). Then, the parylene surface was mildly treated with oxygen plasma to be roughened and treated again with the parylene adhesion promoter. This was done to improve the adhesion of the metal layer on it. After a $0.5 \mu \mathrm{m}$ thick aluminum (Al) layer was sputter-deposited and patterned, a fluoropolymer, Cytop (Asahi Glass, Japan), was used to render the surfaces hydrophobic. Cytop was spin-coated to form a very thin layer $(200-300 \mathrm{~nm})$ and cured for several hours. A low temperature curing process was carried out in an inert atmosphere $\left(\mathrm{N}_{2}\right)$ to avoid sudden degradation of the underlying parylene layer because degradation in the presence of oxygen at an elevated temperature reduces the tensile and dielectric strength of the parylene layer [24]. As illustrated in step (e), the ITO coated cover glass slip with an orifice (250 $\mu \mathrm{m}$ in diameter) was aligned and attached to the device with a $25 \mu \mathrm{m}$ thick SU-8 spacer, which secures a 
gap for the liquid film between the two substrates. The orifice in the cover slip was made via ablation by an excimer laser $(\lambda=266 \mathrm{~nm})$. Immediately after the ablation of the hole in the cover slip, the surface was rendered hydrophobic by the application of a Cytop coating and cured at $180{ }^{\circ} \mathrm{C}$ for an hour. In step (f), the patterned PZT ceramic is bonded to the bottom side of the liquid film driver using a general purpose epoxy with a $200 \mu \mathrm{m}$ thick SU-8 spacer in between. Finally, a thin liquid film was squeezed between the cover slip and the ejector surface with the help of a syringe. The size of the liquid film varies slightly from device to device, but it did not affect the result overall.

(a)

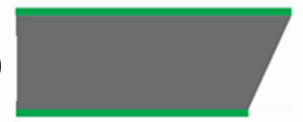

(b)

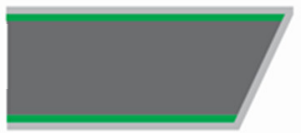

(c)

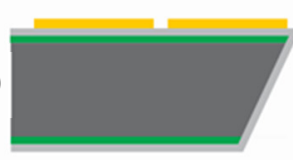

(d)
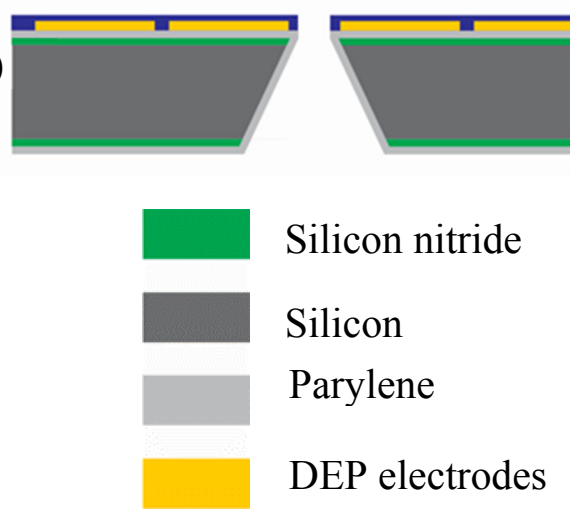

(e)
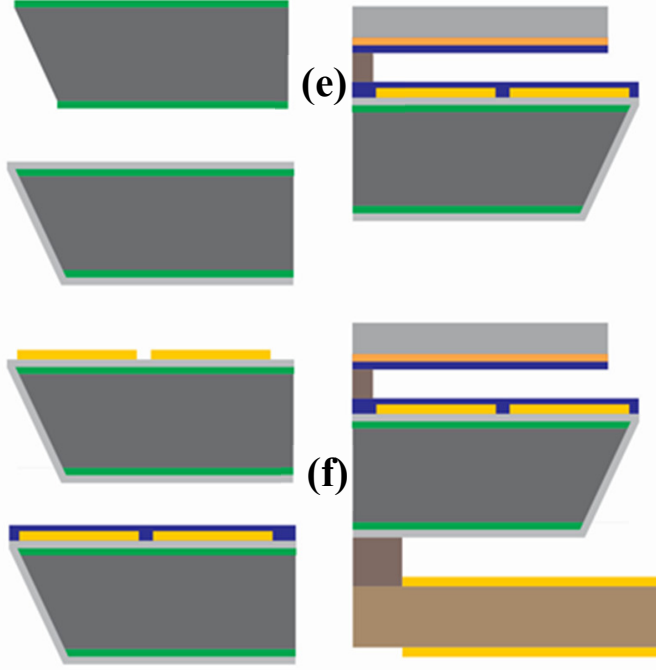

(f)
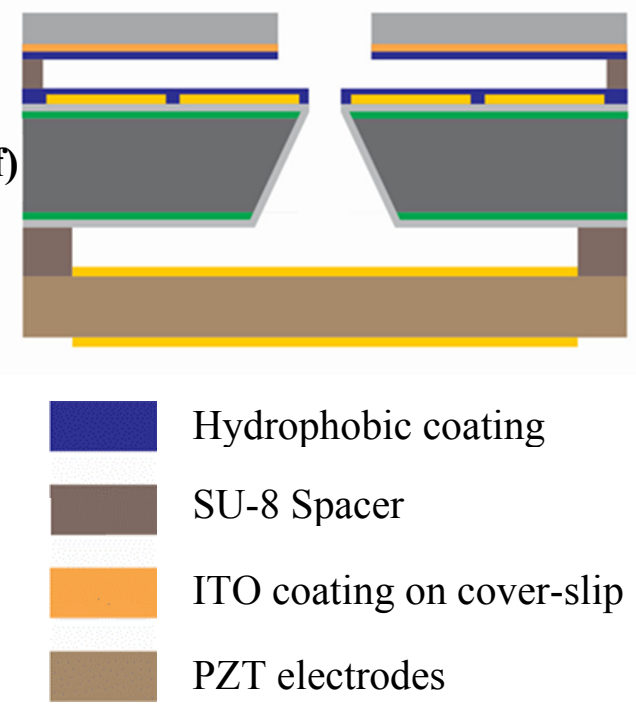
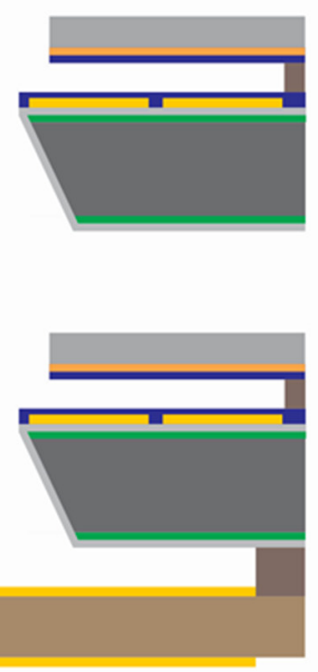

Hydrophobic coating

SU-8 Spacer

ITO coating on cover-slip

PZT electrodes

Figure 4. Fabrication steps. (a) KOH etching of Silicon. (b-e) Fabrication of liquid film driver. (f) Bonding to PZT ceramic. 


\subsection{Study of liquid-core materials for various bio-ink materials}

Several materials were tested to determine the best candidate materials to be used as the micro-fluidic valve material. Six different candidate materials were initially chosen - the different parameters of which have been tabulated in Table I. Different parameters such as dielectric constant of the material, the surface tension, the vapor pressure, the actuation voltage and toxicity were evaluated. The most important criterion of the valve material is its immiscibility with the working fluid. Complete immiscibility of the fluidic valve material over the working fluid is the most important criterion and a liquid with $0 \%$ solubility is preferred over very low solubility level candidate liquid materials. A very low solubility liquid has a tendency for contaminating the working fluid over time depending on the interfacial chemistry occurring between the two liquids. This could also result in the thin liquid film loosing mass to the working fluid over time. Hence complete immiscibility as a desired characteristic was closely evaluated while choosing the microfluidic valve material. The second most important criterion that was paid close attention to while selecting the microfluidic valve material was its vapor pressure. The microfluidic valve material would need to possess a very low to almost negligible vapor pressure so that it can sustain prolonged periods of time in the device without any change in size/volume with time. Any change in the size/volume of the sandwiched liquid droplet would change the actuation behavior. With decreasing size/volume of the sandwiched liquid droplet over time, the actuation behavior would change to a point where the microfluidic valve would fail to transition from one DEP electrode pad to the other and no further transition from the 'open' state to the 'closed' state and vice versa would be possible. This is when complete valve failure occurs. In order to prevent complete valve failure from occurring, it is very 
crucial that the material chosen for the liquid valve has a negligible vapor pressure over a wide range of temperatures. The dielectric constant is another parameter that was looked into. In order to drive the liquid film onto and off the nozzle, electrical actuation is required. Since the driving force exerted on the liquid film is due to dielectrophoresis, there exists a relation between the actuating voltage and the force exerted on the liquid droplet as seen in this equation $F_{D E P}=\frac{\varepsilon_{o}(\varepsilon-1) W}{2 d} V^{2}$ where $V$ is the driving voltage, $W$ is the width of the two parallel plates, $d$ is the spacing between the plates, $\varepsilon_{o}$ and $\varepsilon$ are the absolute and relative permittivity of the liquid. Higher the driving voltage, higher the force exerted. For a given voltage, the driving force would be proportional to the dielectric constant of the liquid film being polarized. For a desirable valve behavior, a lower driving voltage would always be favorable over a higher driving voltage. A material with a higher dielectric constant would possess a higher degree of polarization and hence would require a lower actuation voltage.

TABLE I. A comparison of the different liquids to be used for the liquid film along with the actuation voltage, dielectric constant, solubility in water, surface tension, vapor pressure and toxicity.

\begin{tabular}{|c|l|l|l|l|l|l|}
\hline $\begin{array}{l}\text { Liquid } \\
\text { Valve } \\
\text { Material }\end{array}$ & $\begin{array}{l}\text { Actuation } \\
\text { Voltage }\end{array}$ & $\begin{array}{l}\text { Dielectric } \\
\text { constant } \\
\left.\mathbf{(} \mathbf{2} \mathbf{2 0}^{\circ} \mathbf{C}\right)\end{array}$ & $\begin{array}{l}\text { Solubility in } \\
\text { water } \\
\mathbf{( g m / 1 0 0 m l )}\end{array}$ & $\begin{array}{l}\text { Surface } \\
\text { tension(dy } \\
\text { nes/ cm) }\end{array}$ & $\begin{array}{l}\text { Vapor } \\
\text { pressure } \\
\mathbf{m m H G @} \\
\mathbf{2 5 C )}\end{array}$ & $\begin{array}{l}\text { Toxicity } \\
\text { LD50 (Oral, } \\
\text { rat) }\end{array}$ \\
\hline Mercury & --------- & Nil & immiscible & 486.5 & 0.0018 & -------- \\
\hline Silicone Oil & $350-400 \mathrm{~V}$ & 2.2 & immiscible & 20.6 & $<1$ & $>17 \mathrm{gm} / \mathrm{kg}$ \\
\hline Nitrobenzene & $75 \mathrm{~V}$ & 36.1 & 0.19 & 43.9 & 1 & $349 \mathrm{mg} / \mathrm{kg}$ \\
\hline n-Nonane & $350-400 \mathrm{~V}$ & 1.99 & immiscible & 22.8 & 3.22 & $5000 \mathrm{mg} / \mathrm{kg}$ \\
\hline O-xylene & $425-450$ & 2.56 & 0.017 & 30.1 & 7 & $4300 \mathrm{mg} / \mathrm{kg}$ \\
\hline Furfural & $250 \mathrm{~V}$ & $3.3-5.2$ & 8.3 & 41.9 & 1 & $360.5 \mathrm{mg} / \mathrm{kg}$ \\
\hline
\end{tabular}


In the microfluidic valve testing, it was seen that Nitrobenzene leaves a watermark residue behind on the DEP electrode pads possibly due to electrolysis when used without a dielectric layer. Actuation speed is very fast for $100 \mathrm{VDC}$ and at $75 \mathrm{VDC}$ and is comparable to silicone oil at higher driving voltages.

$\mathrm{n}$-Nonane begins to exhibit actuation via dielectrophoresis at a potential of $350 \mathrm{~V}$ and is fast enough for normal valve mechanism at 400 VDC. This material does not leave any residue or undergo electrolysis even at $400 \mathrm{VDC}$. The high actuation voltage is due to the lower dielectric constant of 1.99 as compared to nitrobenze which can be actuated with voltages as low as $75 \mathrm{VDC}$ and has a dielectric constant of 36.

O-Xylene begins to start actuating via DEP at $425 \mathrm{VDC}$ and at $450 \mathrm{VDC}$ is satisfactory for normal valve functioning. It was observed that the size of the microfluidic valve composed of Xylene begins to reduce in diameter possibly due to loss of mass during actuation by volatilization. Within ten minutes of continuous testing, the diameter of the microfluidic valve was seen to reduce by almost $25 \%$. No residual layer was left behind nor breakdown due to electrolysis was observed.

\subsection{Choice of dielectric layer}

Since some of the liquids that were initially tested for use as the microfluidic valve were conductive in nature, a dielectric layer was implemented on top of the electrode pads to prevent breakdown of the liquid through electrolysis. Without the insulation layer present, some conductive liquids such as certain organic compounds undergo breakdown via electrolysis. Electrical breakdown of the microfluidic valve material is not desired as this could severely affect the performance of the valve mechanism. 
TABLE II. The different dielectric materials that were used on the ejector surface along with the dielectric constant, breakdown voltage and film thickness.

\begin{tabular}{|l|c|c|c|}
\hline \multicolumn{1}{|c|}{ Dielectric material } & $\begin{array}{c}\text { Dielectric } \\
\text { constant }\left(\boldsymbol{\varepsilon}_{\mathbf{r}}\right)\end{array}$ & $\begin{array}{c}\text { Breakdown voltage } \\
(\mathbf{V} / \mathbf{m i l})\end{array}$ & $\begin{array}{c}\text { Film thickness } \\
(\boldsymbol{\mu m})\end{array}$ \\
\hline Parylene C & 3.15 & 6800 & $0.5-1$ \\
\hline SU8 2000 & 4.1 & 2845 & $\sim 10$ \\
\hline Cytop & $2.0-2.1$ & 2286 & $\sim 1$ \\
\hline Anodized Tantalum Pentoxide & $21-23$ & 15000 & $\sim 0.1$ \\
\hline
\end{tabular}

Out of the four dielectric materials that were implemented, Parylene C followed by SU8 proved to be the best. Parylene $\mathrm{C}$ as a dielectric layer resulted in more conformal coatings as compared to SU8 which leaves an edge bead at the edge of the samples. The downside is that such coatings need an adhesion promoter before application. A-174 which is a silane is used to provide better adhesion of Parylene to the substrate. Before Parylene deposition, the samples are coated with the silane solution in a liquid phase, dried and then kept in the deposition chamber. Also when Parylene is used, thermal annealing of the hydrophobic (Cytop) layer is restricted to not more than $125^{\circ} \mathrm{C}$ in an inert atmosphere as compared to annealing the fluoropolymer at the recommended $180^{\circ} \mathrm{C}$ as done for most other materials. This is because Parylene undergoes thermal degradation from $120{ }^{\circ} \mathrm{C}$ onwards via oxidation. Since this dielectric material is deposited in the vapor phase, it conformally coats all the microstructures that may be present on the substrates unlike other spin on dielectrics such as spin-on glass or SU8. Cytop can also be used as a dielectric layer when conductive microfluidic valve materials are to be used. In order to obtain a $1 \mu \mathrm{m}$ thick film, a $2 \%$ Cytop solution needs to be spin coated 5-6 times with short baking between each spin. The resultant films are smooth. Such dielectric films of Cytop also serve as a hydrophobic layer and no additional hydrophobic coating is required. Tantalum pentoxide films which are dielectric are formed by anodization of sputter deposited tantalum films. Such metallic 
films are patterned first, thereby forming the DEP electrodes and then only the part that needs to be coved by the dielectric material is anodized in an anodizing solution. The anodizing process is a cumbersome one as all isolated electrodes need to be electrically connected before anodization and hence is not preferred.

\subsection{Experimental setup}

\subsubsection{The strobing circuitry}

Observation of ejected droplets is a very complicated process as the droplets produced by a micro-ejector are very small $(\sim 30-50 \mu \mathrm{m})$ and travel at high speeds once ejected $(\sim 5-20$ $\mathrm{m} / \mathrm{s}$ ). In order to observe the ejected droplets, short laser as well as LED light pulses are used for back illumination for the visualization and recording of liquid micro-jet disintegration. The strobing circuitry used to capture the ejected droplets has been presented in Figure 5 and has been implemented from a similar circuit used for strobing [25].
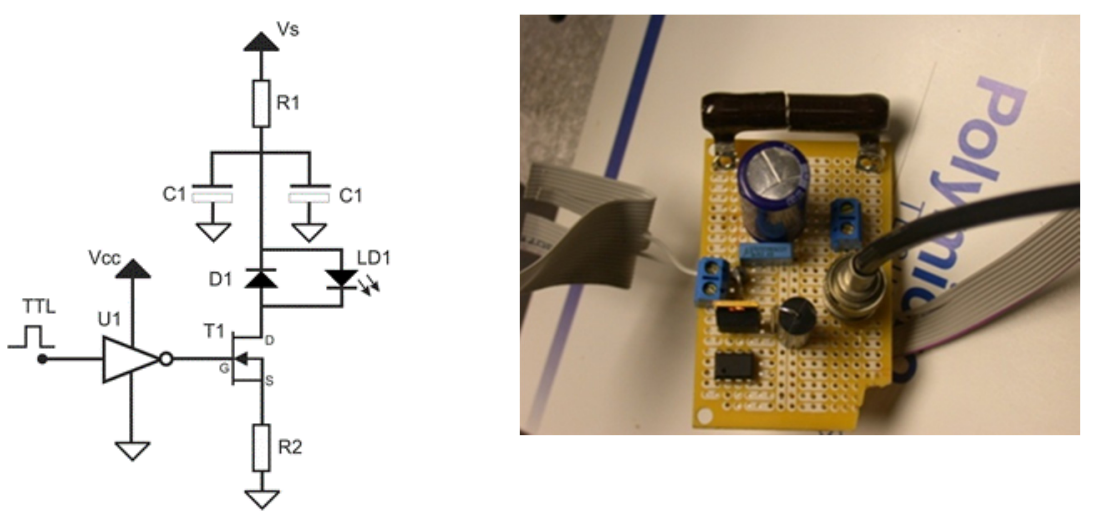

Figure 5. Strobing circuitry: Typical values $-R 1 \sim 1-2 \Omega ; R 2=0.02 \Omega ; C 1=1 \mu F$ poly; $C 2=2200 \mu F ; D 1=B Y T 01-400 ; T 1=I R F B 3206 G P b F ; U 1=U C C 37322 P$

One of the main characteristics of a strobing circuitry is that it needs to provide a high current over a short duration. Typical 'ON' times range from 1-5 $\mu$ s. This designed circuit 
(Figure 5) acts as a switched current source to the load which is the high power LED. The capacitors are charged through the power supply $(\sim 25 \mathrm{~V})$. The MOSFET power transistor (T1) is triggered via a MOSFET driver circuit (U1) which has a TTL compatible input. The LED cathode is connected to the drain of the power transistor whose source is connected to ground via a low resistance power resistor (R2, typ. 0.01-0.1 $\Omega$ ). The LED is protected from reverse currents that arise during the rapid switching transients of the circuit by diode D1. LED's operated in continuous mode can be detrimental and hence R1 acts as an over current protection. It also limits the charging current of the capacitors in between the current pulses [25].

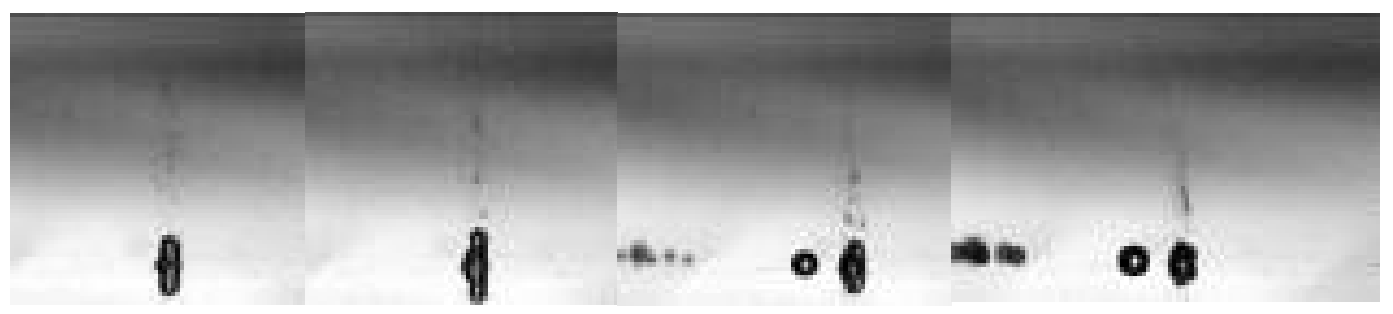

Figure 6. Images of ejected ink droplets using the new strobing circuitry.

Using this new strobing circuitry, very high contrast images can be obtained using backillumination as seen in the images shown above (Figure 6). 


\subsubsection{High speed imaging technique for the ejector}

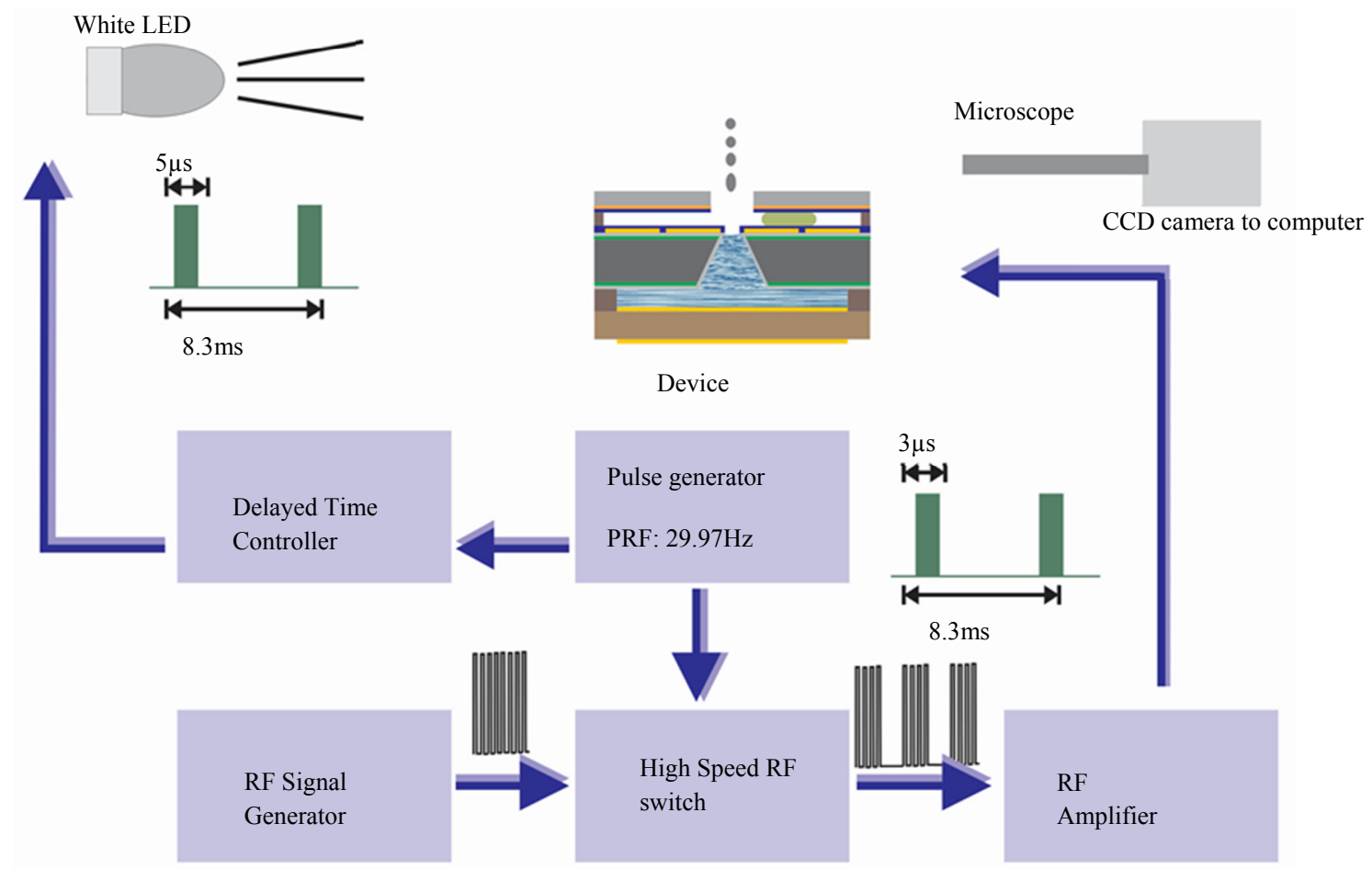

Figure 7. Schematic of the test setup with short pulse stroboscopic illumination to image ejected droplets

The microfluidic ejector with the LiFE microfluidic valve was tested first with ultra-high purity de-ionized (DI) water. The fluidic chamber in the ejector system was primed with DI water delivered by a syringe pump. A schematic of the experimental setup used for the visualization of the ejection process is illustrated in Figure 7. Because droplets are ejected very quickly and travel very fast, the motion of the ejected droplets was captured stroboscopically. A $1 \mathrm{Vp}-\mathrm{p}$ sinusoidal signal at around $2 \mathrm{MHz}$ was fed into an RF switch and modulated with a $29.97 \mathrm{~Hz}$ signal having a pulse width of $3 \mu$ s to synchronize with the frame rate of the CCD camera. A digital delay/pulse generator (Stanford Research Systems DG535) was triggered externally at $29.97 \mathrm{~Hz}$ using a waveform generator (HP 33120A), which was used to precisely control the pulse widths and the timings of the signals sent to the strobe circuit and the ejector. The modulated signal was then amplified by an RF 
amplifier (Amplifier Research 150A100A) to run the piezoelectric-based microfluidic ejector. A standard CCD camera connected to a microscope was used to capture the images. Individual frames were later analyzed through an image acquisition system.

\subsection{Results}

\subsubsection{Testing of the liquid-core microfluidic valve}

After closely evaluating all the candidate materials, silicone Oil was chosen as the nonaqueous liquid for the microfluidic valve as it is one of the most inert and immiscible liquids with water. Assuming most inks as well as biological fluids are aqueous based, silicone oil would be highly immiscible with such working fluids. Silicone oil having a dielectric constant of 2.71 and a dielectric strength of $390 \mathrm{KV}$ (100 mil gap) was chosen because of its immiscibility and highly inert nature with most water based pigment inks and biological reagents. Silicone oil has a very low electrical conductivity of less than $10^{-}$ ${ }^{10} \mathrm{~cm} / \Omega$ which makes it ideal in DEP devices where no dielectric layers are used as in EWOD devices. This also prevents it from undergoing electrolysis unlike most other conductive organic liquids that need a 'dielectric layer protection' in order to prevent them from undergoing electrolysis during actuation in microfluidic devices. The only disadvantage in using silicone oil is its high DEP actuation voltage required for droplet movement. In our devices, the oil droplet is actuated with a voltage as low as 400 VDC. Hermetic nozzle sealing is achieved by actuating the oil droplet onto the electrode with the central hole and above the nozzle opening in the silicon wafer. 

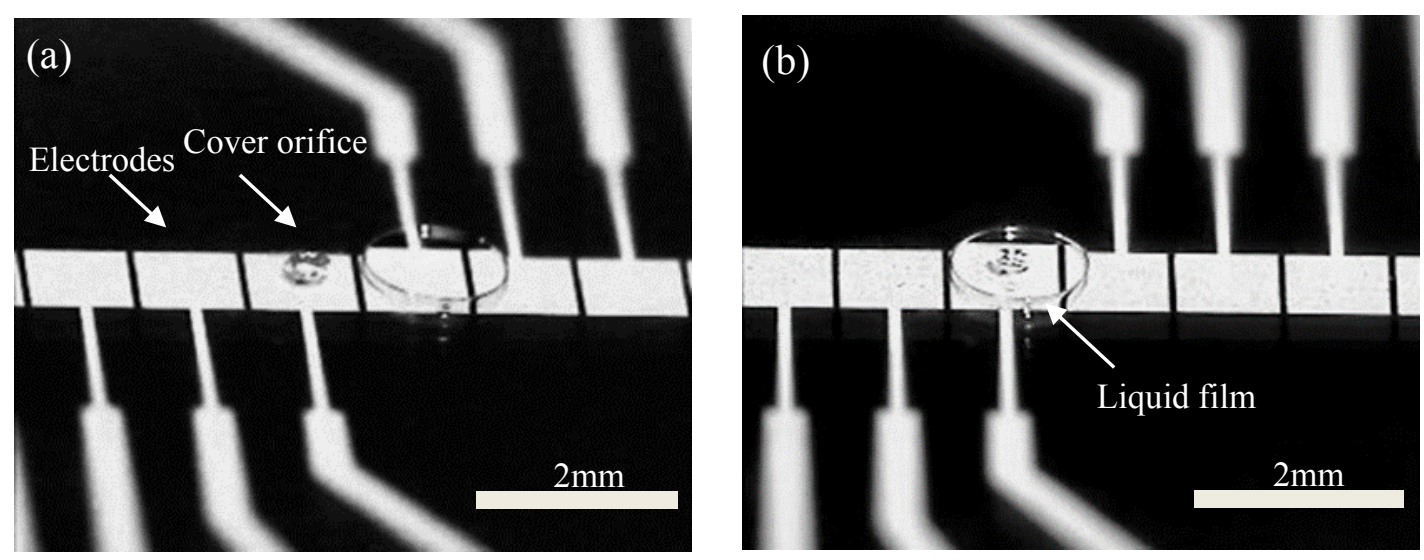

Figure 8. Images of a LiFE microfluidic valve using silicone oil in (a) 'open' position and (b) 'closed' position. The liquid film moves away and onto from the ejector nozzle.

\subsubsection{Evaporation controllability}

The efficacy of the thin liquid film (valve-core) for the evaporation control was tested with de-ionized (DI) water and a general purpose aqueous ink as the working fluid. Quick evaporation related nozzle failures have been observed in the aforementioned ejector when the LiFE valve was not utilized. It was observed that dried and solidified ink pigments completely clogged the fluidic pathway at the nozzle area when the nozzle had been left open for an extended period of time. In our previous studies, it was seen that the nozzle had clogged within 30 minutes when a general purpose ballpoint ink had been used. The relatively viscous ballpoint ink (even after dilution with DI water) tends to dry out quickly and solidifies. In recent testing, a general purpose fountain pen ink and DI water were used separately as the working fluids. No additives such as viscosity or surface tension modifiers were used when testing with DI water. With the microfluidic valve left open, the nozzle was clogged within $6 \mathrm{hrs}$ and no further ejection was possible even with increased amplitudes of the driving voltage with ink as the working fluid. However, even after a long period (120 days) of the 'closed-valve' mode, ejection could still be resumed. It was very 
obvious that the fluidic loss via evaporation occurred only through the nozzle (exposed to the air for the extended period of time) and led to the nozzles being clogged. When DI water was employed as the working fluid, we found that the ejection stopped soon after evaporation lowered the fluid level down and led to the formation of an air pocket inside of the nozzle. For quantitative analysis, we have tried to measure the evaporation rate through the nozzle by monitoring the growth rate of the air pocket in the ejector. For this, a transparent glass plate was attached at the bottom of the ejector in place of the piezo ceramic so that any evaporation related phenomenon could be visually observed. As shown in the images in Figure 9, when the nozzle (50 $\mu \mathrm{m}$ X $50 \mu \mathrm{m})$ was left open, the volume of the working fluid (DI water) in the reservoir decreased over time due to evaporation. While the ejector was not operating, the meniscus at the nozzle lowered continuously due to the quick evaporation of the working fluid. Eventually, air entered the reservoir through the nozzle and formed an air pocket. From the size growth of the air pocket with time, the evaporation rate through the nozzle was calculated to be about $60 \mathrm{nl} / \mathrm{hr}$. On the other hand, when the nozzle is closed by the LiFE valve, evaporative fluidic loss in the reservoir was not noticeable even after extended periods of time ( $>120$ days). As shown in the graph in Figure 9, the evaporation problem through the open liquid-air interface is entirely eliminated and well controlled by utilizing the LiFE microfluidic valve. 


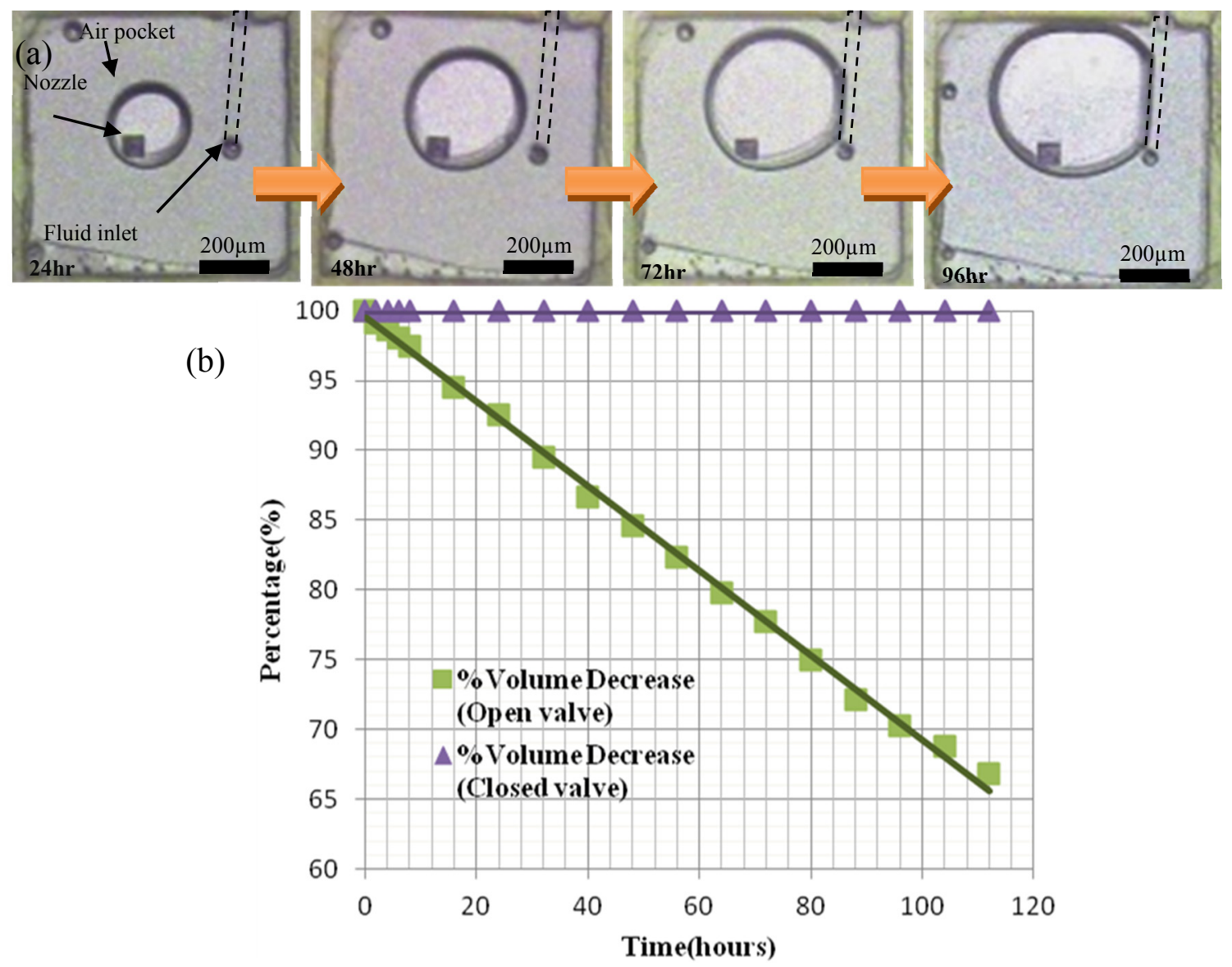

Figure 9. (a) Images of growing air pocket size due to the quick evaporation through the nozzle when the microfluidic valve is in the 'open' state. (b) Graph of liquid reduction data when the valve was left open or closed for a while.

Moreover, the evaporation controllability over the evaporative aqueous-based ink which causes serious clogging problems have been studied. With the LiFE valve left in the 'open' position continuously for about 6 hours, the droplet ejector could not resume ejection any longer. It was observed that the nozzle area dried up completely and the meniscus of the ink could not be seen at all. However, with the LiFE valve in the 'closed' position immediately after ejection, the nozzle condition remained fresh and ejection was resumed immediately. Even after 120 days in the closed mode, stable ejection was still observed with a clean nozzle condition. As compared in Table III, it has been deduced that the quick 
evaporation rate of the working fluids through the tiny nozzle was very well controlled when the LiFE microfluidic valve was in use. When the oil film covers the nozzle area completely, microscopic evaporation of the working fluids (water and ink) through the tiny nozzle hole was very well prevented and any volume reduction was entirely eliminated.

TABLE III. Evaporation speed data when the LiFE microfluidic valve is opened or closed

\begin{tabular}{|c|c|c|}
\hline \multirow{2}{*}{$\begin{array}{c}\text { LiFE Microfluidic } \\
\text { Valve }\end{array}$} & D.I. water & Aqueous based Ink \\
\cline { 2 - 3 } Closed & N/A ( $>120$ days $)$ & N/A ( $>120$ days $)$ \\
\hline Open & $\sim 24 \mathrm{hr}$ & $\sim 6 \mathrm{hr}$ \\
\hline Failure Mechanism & air pocket & Crystallization / coagulation \\
\hline
\end{tabular}

\subsubsection{Bio-ink delivery}

Several different bio-fluids were tested in the ejector integrated with the microfluidic valve including various hydrogels and protein solutions. Some testing on the long term effects of the compatibility of the bio-fluids with the valve material - silicone oil, was also done. It should be noted that the ejection of bio-fluids poses a greater challenge as compared to simple aqueous solutions. Since bio-fluids, in general, have lower surface tensions than pure de-ionized water (72 dynes/cm), it was observed that certain bio-fluids such as hydrogel solutions have a tendency of seeping out of the nozzle and fouling the area of the ejector surface around the nozzle. Furthermore, the contamination and accumulation of bio-material around the ejector nozzle can cause undesirable effects. Some of these include 
unstable ejection, generation of satellite droplets, non-unidirectional ejection and eventual clogging even while being safeguarded against evaporation with the microfluidic valve.

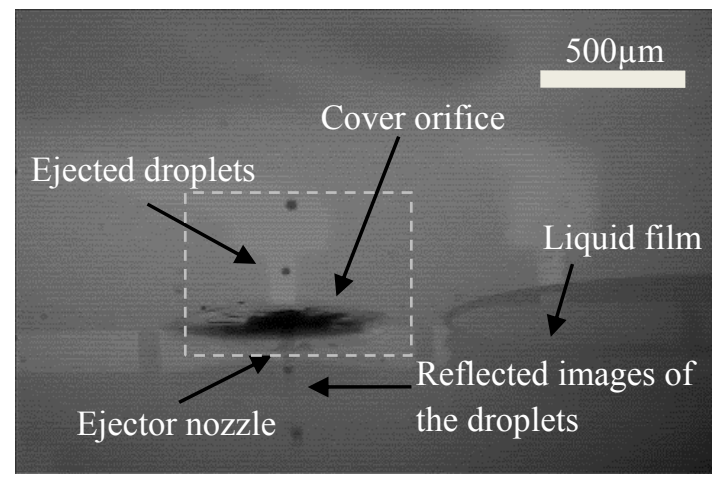

(a)

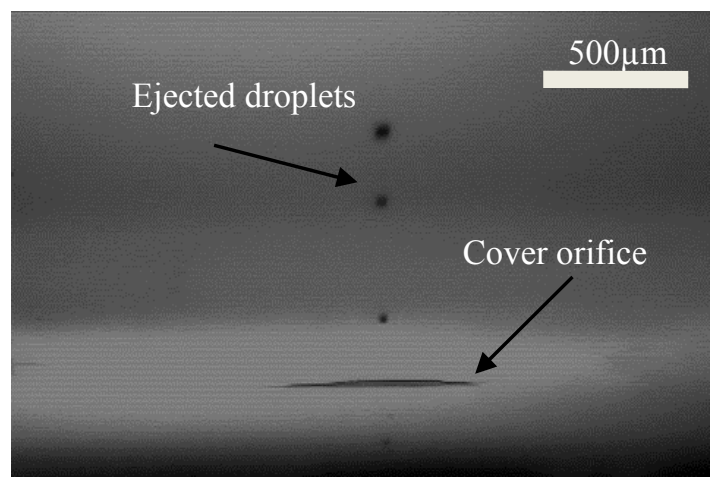

(b)

Figure 10. Images of ejector with the LiFE microfluidic valve. (a) Ejected droplets pass through the cover orifice while the LiFE valve is left open. (b) Magnified view of the cover orifice area. A 10\% Polypropylene gycol (PPG) MW 200 solution has been used as the ejection fluid to improve ejection.

As can be seen in the above images in Figure 10, liquid droplets of a bio-fluid with an average diameter of $30-50 \mu \mathrm{m}$ are seen ejecting out of the nozzle in a very well orderly manner without the formation of any satellite droplets. The ejection of hydrogel solutions such as Gelatin, Agarose, and Collagen had successfully been tested and is described in the next section. Testing protein solutions which had been the next task was also carried out with efforts targeting a broad range of protein types. To begin, the ejection of Streptavidin which is a $60 \mathrm{kDa}$ protein purified from the bacterium Streptomyces avidinii was successfully demonstrated. Streptavidin which has an extraordinarily high affinity for biotin, is widely used in bio-engineering applications. Apart from Streptavidin, various other proteins were also tested in the ejectors. 


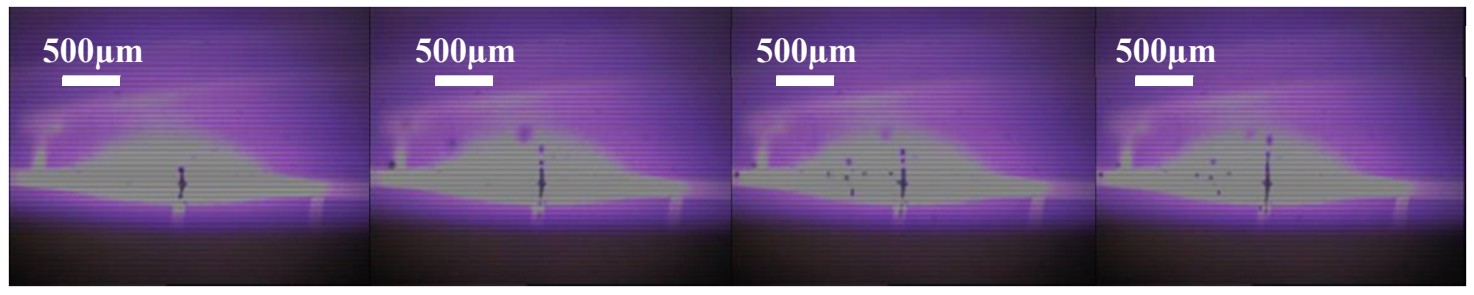

Figure 11. Ejection of images of protein streptavidin without the initial top coverslip for determining the excitation amplitude and pulse width of the ejector.

The ejection of protein solutions too, like other bio-fluids, can be a challenge depending on the nature of the protein being ejected. All the testing of proteins listed in Table IV, using the ejector was done initially without the microfluidic valve and the top cover-slip in order to determine the various ejection parameters. Since proteins can either have globular or fibrous structures, it was observed that the ejection characteristics were protein dependent. It was also observed that the ejection of globular protein solutions as compared to fibrous ones seemed easier. Once the ejection parameters for a particular protein had been determined, the cover slip was assembled and the microfluidic valve was employed in the device.

TABLE IV. All the proteins that were tested in the ejector with the microfluidic valve

\begin{tabular}{|c|c|c|c|c|}
\hline Protein & Concentration & $\begin{array}{c}\text { Microfluidic } \\
\text { valve } \\
\text { compatibility } \\
\text { (Silicone oil) }\end{array}$ & $\begin{array}{c}\text { Ejection } \\
\text { in } \\
\text { droplet } \\
\text { ejector }\end{array}$ & Comments \\
\hline Streptavidin & $100 \mu . g m / m l$ & $\checkmark$ & $\checkmark$ & Stable ejection \\
\hline $\begin{array}{c}\text { Creatine } \\
\text { Phosphokinase }\end{array}$ & $100 \mu . \mathrm{gm} / \mathrm{ml}$ & $\checkmark$ & $\boldsymbol{x}$ & Very unstable \\
\hline Collagenase & $1 \mathrm{~m} . \mathrm{gm} / \mathrm{ml}$ & $\checkmark$ & $\checkmark$ & $\begin{array}{c}\text { Not so stable } \\
\text { ejection }\end{array}$ \\
\hline Urease & $100 \mu . \mathrm{gm} / \mathrm{ml}$ & $\checkmark$ & $\checkmark$ & Stable ejection \\
\hline Arginase & $100 \mu . \mathrm{gm} / \mathrm{ml}$ & $\checkmark$ & $\checkmark$ & Stable ejection \\
\hline Avidin & $100 \mu . \mathrm{gm} / \mathrm{ml}$ & $\checkmark$ & $\checkmark$ & Stable ejection \\
\hline Cellulase & $100 \mu . \mathrm{gm} / \mathrm{ml}$ & $\checkmark$ & $\checkmark$ & $\begin{array}{c}\text { Not so stable } \\
\text { ejection }\end{array}$ \\
\hline
\end{tabular}


All the proteins that were tested in the ejectors have been shown in Table IV. A solution concentration of $100 \mu . \mathrm{gm} / \mathrm{ml}$ with de-ionized water was used in all the cases. With the exception of 'Creatine Phosphokinase', all the proteins showed stable ejection as described in Table IV. It is not fully understood why certain proteins such as 'Creatine Phosphokinase', which is a globular protein could not be stably ejected.

One advantage in using this technology - the ejector implemented with the microfluidic valve, for delivery of bio-fluids such as protein solutions is that even miniscule quantities can be well preserved inside the ejector over extended periods of time. Similar to the previous results of evaporation controllability for de-ionized water and ink, the protein solutions could still be ejected after a week, while remaining immune to evaporative effects, with the aid of the microfluidic valve.

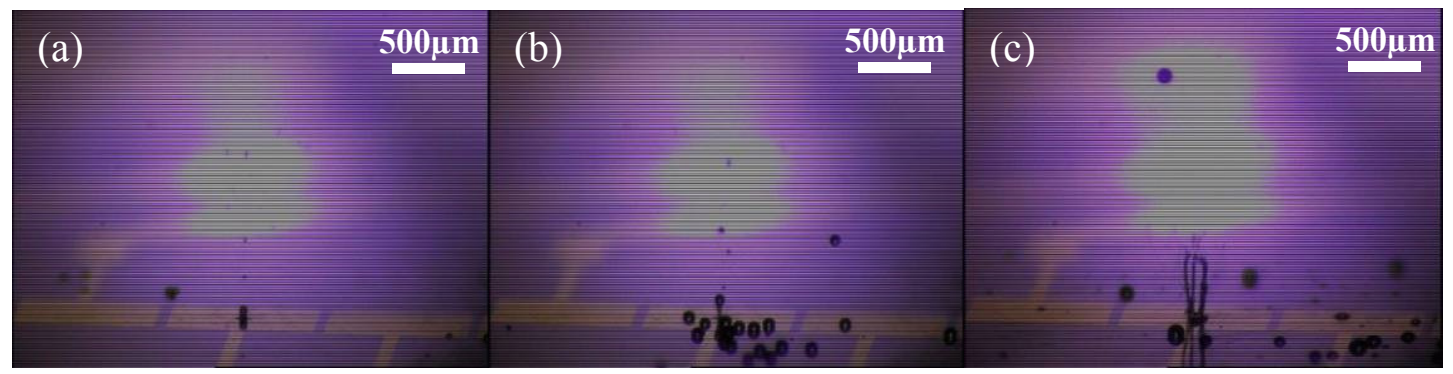

Figure 12. (a) and (b) Ejection testing with the use of viscosity modifiers for improved ejection. A 5\% (wt\%) Poly Ethylene glycol (PEG) MW 200 has been used as a viscosity modifier with water. (c) The onset of clogging due to particulate buildup at the nozzle.

The use of viscosity modifiers such as Poly Ethylene glycol (PEG) and Di-propylene glycol as well as surface tension modifiers such as Triton-X surfactants, added to the fluids to be ejected was also preliminarily investigated during that time. An improved ejection quality with viscosity and surface tension modifiers was observed as seen in Figure 12a and Figure 12b. A $5 \%(w t \%)$ Di-Propylene glycol was preferred over PEG due to the more stable ejection that was achieved. Viscosity modifiers such as PEG and other glycols are desirable 
for an improved ejection quality but would need further evaluation for assessing their toxicological effects on bio-fluids such as proteins and hydrogel solutions.

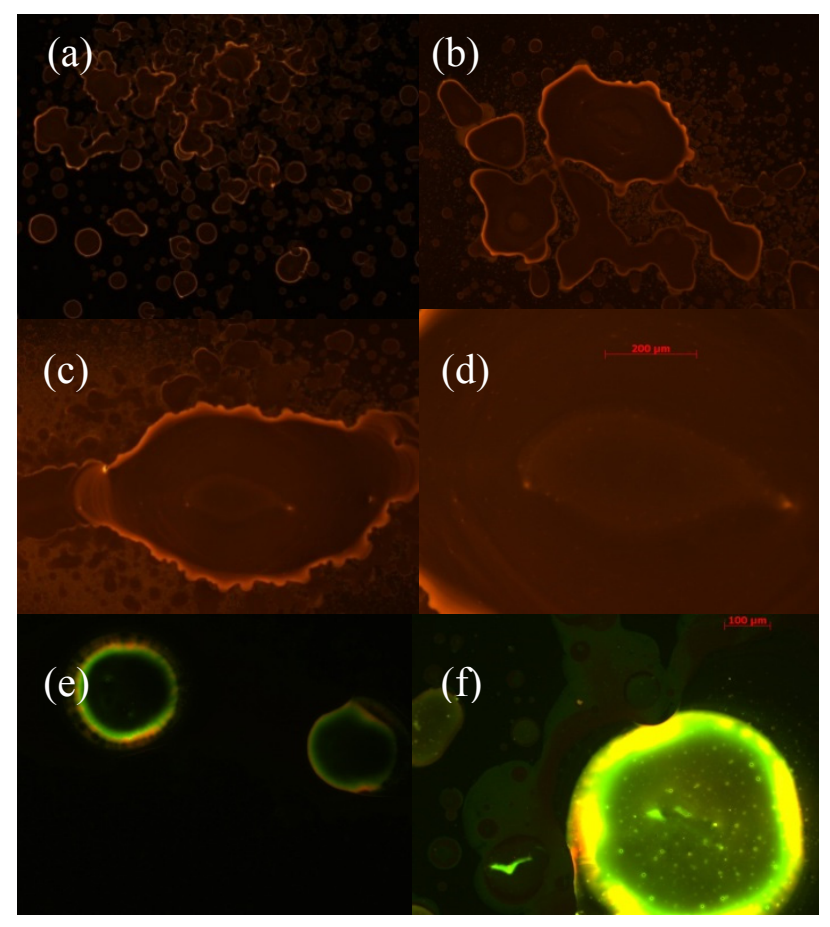

Figure 13. Ejected hydrogel (a-d:gelatin, e,f:collagen) with Rhodamine $6 G \&$ Acridin orange on glass slides using Fluorescence microscope.

Aqueous solutions of naturally occurring hydrogels such as Gelatin, Agarose and Collagen were also tested in the ejectors. These protein and carbohydrate based polymers are used in the design and fabrication of organic scaffolds in skeletal tissue engineering. Clogging is caused when the polymers in the aqueous solutions begin to crosslink and transform into hydrogel networks when solvent evaporation occurs. The ejected hydrogels on glass substrates were imaged using fluorescence microscopy as shown in the Figure 13. As can be seen in Figure 13, fluorescence microscopy revealed droplets of different sizes including larger agglomerates of greater than $500 \mu \mathrm{m}$. Size of single ejected droplets ranged between 10-30 $\mu \mathrm{m}$. All ejection tests were repeated after every few hours to check the durability of 
the hydrogel ejectors by closing the microfluidic valve between tests thereby preventing evaporation. Larger sized features as seen in the above images were due to the overlap of single ejected hydrogel droplets. Once ejected from the nozzle and upon reaching the target coverslip, the ejected hydrogel droplets began to crosslink at a faster rate due to the loss of liquid content through evaporation which is similar to the drying mechanism as seen in commercial inkjet printers. Also, since no cross-linking agents that cause instantaneous hydrogel cross-linking, were used on the substrate, overlapped ejected droplets simply coalesce into larger diameter features. Further imaging and surface characterizations of the substrates onto which the hydrogel droplets are ejected would be required to determine the vertical growth of ejected features. Since during these tests, neither the substrates nor the piezo ejectors were connected to X-Y manipulation stages, ordered placement of ejected hydrogel droplets in defined paths required for 3D hydrogel scaffolds could not be achieved. However in recent testing, printing of the same hydrogels was carried out but in a more orderly manner using the latest clog-free ejector prototype. The details of this clogfree ejector prototype will be discussed in a later chapter of this dissertation.

\subsection{Conclusion}

In Summary, a microfluidic valve based on an immiscible and non-volatile liquid film as a valve-core was developed. The microfluidic valve was used to successfully prevent evaporation of working fluids through microscopic ports. Many immiscible fluids to various aqueous solutions were tested to determine the most suitable candidate material to be used as the valve-core. Finally, an environmentally friendly immiscible fluid - silicone oil, was used as the valve-core material. By moving the thin liquid film to cover or uncover a tiny microfluidic port with a liquid-air interface, microscopic evaporation through the 
port has been very well controlled. Liquid dielectrophoresis (LDEP) technique was used as the actuation mechanism to open and close the fluidic valve. It has clearly been shown that the fluidic loss of the working fluid by quick evaporation as well as clogging due to residue left by evaporation, can be stopped by employing the LiFE microfluidic valve. Furthermore, reliable ejection of bio-fluids such as hydrogels and protein solutions over long periods of time without any clogging problems has also been demonstrated. This finding has immense potential in the prevention of clogging problems in various microfluidic systems having air-exposed microfluidic ports.

The liquid-core microfluidic valve was successful demonstrated as an effective evaporation barrier in this chapter. Since then, efforts were focused on increasing the reliability of the microfluidic valve. Since the microfluidic valve is free to move in any direction on the 2D plane of the ejector surface, its reliability can be compromised when it leaves the electrode pads that actuate it onto and off the nozzle. Unconstrained motion can hinder the microfluidic valve's functionality while rendering its spatial controllability by the electrodes ineffective. However, this is prevented by confining or restricting its motion along certain paths on the ejector surface. For this purpose, certain liquid repellent features were embedded on the ejector surface that restricted the motion of the microfluidic valve to a single dimension. The next chapter discusses in detail how the motion of low surface tension liquids, such as the microfluidic valve, can be well constrained along certain paths on a surface. 


\section{Chapter 3}

\section{LOW SURFACE TENSION LIQUID CONFINEMENT USING OIL-REPELLENT SURFACES}

\subsection{Oil-repellent surfaces and virtual walls for guiding low surface tension liquids}

Confining and manipulating non-aqueous low surface tension liquids, such as the microfluidic valve material, within a desired area, are not trivial and pose great challenges in microfluidic devices. In this section, newly developed oil-repellent surfaces formed by using a plasma assisted surface micromachining process have been employed to control the surface wettability for low surface tension liquids. While exploring these surfaces further, it was realized that the plasma assisted patterning of these surfaces could be used for the transport of low surface tension liquids such as the microfluidic valve liquid along desired pathways.

It is a known phenomenon that liquid streams in microfluidic devices, supported by the liquid-air interface at two opposite sides, are said to be supported by virtual walls. During that time, it was discovered that the newly developed oil-repellent surfaces could be used for the formation of virtual walls for supporting low surface tension liquids such as dodecane, hexadecane etc. very effectively. In this manner, the liquid-core material of the microfluidic valve can be confined along certain paths using virtual walls. Previously, the guiding and confinement of aqueous liquids along pre-defined paths using virtual walls had been demonstrated by several groups. This was simply accomplished by altering the effective surface energy along the desired paths. While this technique is excellent for the guiding of aqueous liquids, non-aqueous low surface tension liquids such as the microfluidic valve cannot be confined using simple alterations in surface energy. 


\section{Conventional Superoleophobic surfaces (side view)}

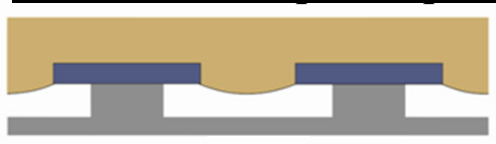

(a)

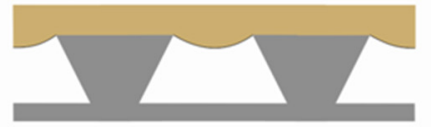

(b)

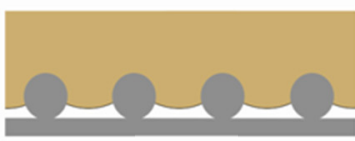

(c)

\section{Oil-repellent surfaces (side view + top view)}

(d)

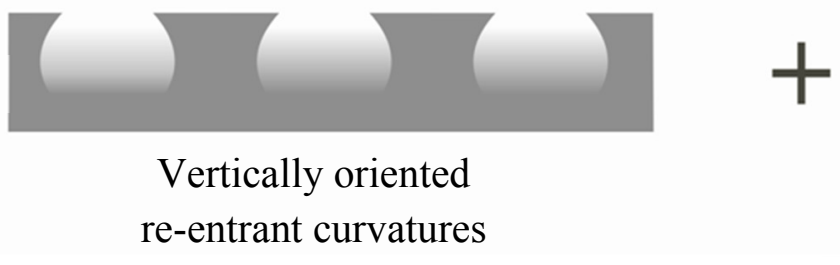

(e)

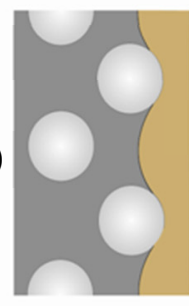

Top View

Laterally oriented re-entrant geometries

Figure 14. Illustration of the conventional superoleophobic surfaces (a-c). Oil can be pinned on (a) mushroom-like structures, (b) sharp edges and (c) bump-like structures without wetting the entire surface. The newly developed oil-repellent surface has (d) reentrant curvatures oriented in the vertical direction as well as (e) re-entrant circular geometries oriented in the lateral direction.

Superoleophobic surfaces were previously explored for this purpose. However, conventional superoleophobic surfaces generally exhibit excellent repellence to vertically approaching liquid droplets as seen from sessile droplet contact angle measurements [2631]. It has been well characterized that the re-entrant and overhanging mushroom-like structures in superoleophobic surfaces as shown in Figure 14 (a-c) are able to keep the vertically approaching low surface tension liquids from penetrating the patterned cavities on the surfaces [26, 29-32]. An oil droplet contacts and gets pinned on the surface of individually isolated and elevated tiny islands having re-entrant structures underneath (Figure 14a). The presence of the air pockets under the oil droplet and between the reentrant structures helps the surface become superoleophobic. Interestingly, the surface chemistry of the superoleophobic surfaces is actually slightly oleophilic [33]. The oil droplet can easily wet a fraction of the surface area of the re-entrant structures but does not penetrate the air pockets. However, the stability of the liquid-air boundary is not very 
strong and the oil can easily spread laterally when forced by external pressure [32] or be perturbed by electric fields $[32,34]$, and readily make a transition into the Wenzel state. Micro/nano structured superhydrophobic surfaces prevent lateral spreading of aqueous solutions due to the inherently hydrophobic surface chemistry [33]. On the other hand, conventional superoleophobic surfaces cannot effectively contain lateral spreading of a liquid droplet in the Wenzel state by pinning the contact line.

Patterned micro/nano structured superhydrophobic surfaces can be used to confine and guide aqueous based liquid streams along hydrophilic paths $[35,36]$ but conventional superoleophobic surfaces such as mushroom structured surfaces do not exhibit similar confinement to low surface tension liquids. Through experimental observations, we found that when an oil droplet laterally transitions from an oleophilic area onto the superoleophobic area, it can easily wet the superoleophobic area because its three phase contact line (TCL) cannot be pinned (Figure 15). This clearly evidences the difficulty to confine low surface tension liquids such as the microfluidic valve within a predefined boundary by conventional superoleophobic surfaces. In previous works however, another method for creating superoleophobic surfaces was introduced by electrospinning blends of fluorodecyl polyhedral oligomeric silsesquioxane (POSS) and poly(methyl methacrylate) (PMMA) polymers [28]. Although this technique exhibits excellent superoleophobicity, patterning these surfaces to produce well defined microfluidic paths using conventional photolithography seems challenging. 


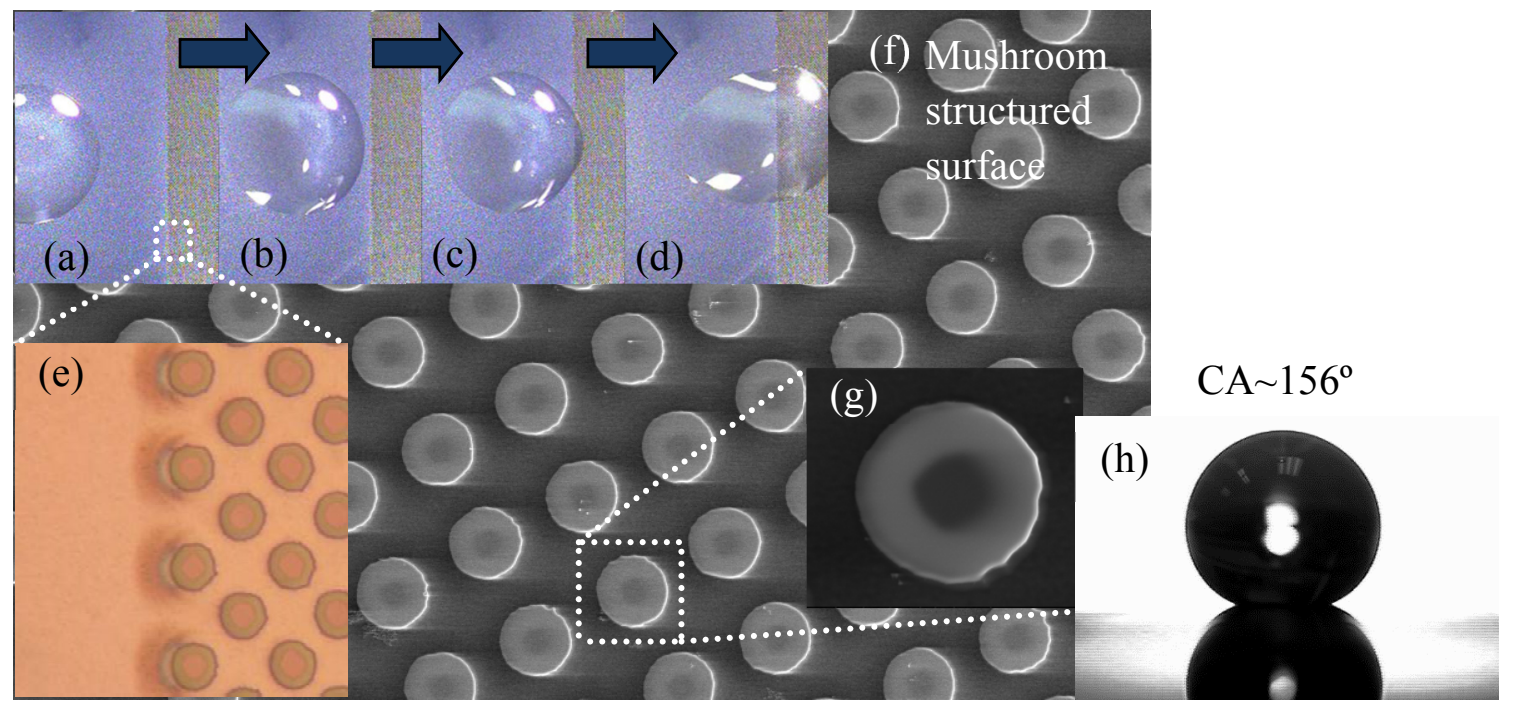

Figure 15. Images of a silicone oil droplet (microfluidic valve) sliding $(a \rightarrow b \rightarrow c \rightarrow d)$ from a flat (oleophilic) region towards a superoleophobic region by gentle pneumatic perturbation. The mushroom-like structures in the conventional superoleophobic surface easily get wetted $(c, d)$ by the oil droplet when brought in contact from the lateral direction. (e) Optical microscope image of the boundary between the flat oleophilic and superoleophobic region. ( $f$ and $g$ ) SEM images of the mushroom-like structures of the superoleophobic surface. (h) Image of a contact angle measurement for a hexadecane droplet on the superoleophobic surface.

Control of surface wettability for manipulating liquids has been widely adapted in many practical applications [37]. Although manipulating and controlling aqueous solutions are relatively easy, it is very difficult to prevent spreading of non-aqueous low surface tension liquids (oils including decane, octane, and hexadecane) such as the microfluidic valve liquid onto non-desired regions. In addition, superoleophobic surfaces inside microfluidic channels have not been broadly explored yet. Moreover, inherently oleophobic $\left(\theta>90^{\circ}\right.$ on a smooth surface) surface chemistries are naturally unavailable. This means that the Wenzel state lowers the contact angle further, while the Cassie state is inherently unstable on most geometries [38]. Unlike superhydrophobic surfaces, superoleophobic surfaces cannot be easily fabricated with nano-structures [29] as done in the case for superhydrophobic surfaces. And thus the use of micro/nano-structured features to prevent 
lateral spreading of low surface tension liquids has not been very effective. Furthermore, the relationship between oleophilic surfaces and superoleophobic surfaces is more complex than the relationship between hydrophilic and superhydrophobic surfaces [38]. In fact, the surface chemistry of previously reported superoleophobic surfaces using various fluorinated coatings over re-entrant geometries is actually slightly oleophilic [38]. When the oil drop falls into the Wenzel state due to perturbation, the oil drop can simply be pinned or spread laterally. Thus, it is not clear how oleophobically stable the surface is to lateral flows. In an effort to confine the movement of the microfluidic valve on the ejector surface, we have developed an oil-repellent surface which can resolve the concerns described above. The oil repellent behavior arises most importantly from the interaction of the threephase contact line (TCL) of the thin liquid film / microfluidic valve with the re-entrant geometries of the micromachined features on the surface.

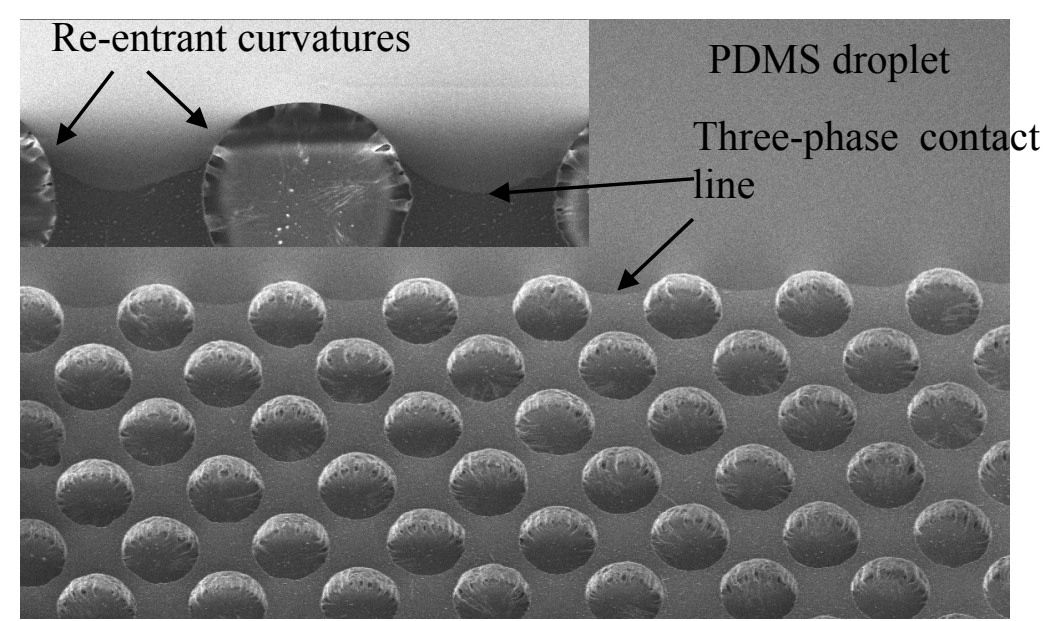

Figure 16. PDMS droplet at the edge of an oil-repellant surface for mimicking the behavior of the oil droplet three-phase contact line at these features.

A study of the interaction of the oil droplet's / microfluidic valve's three-phase contact line with the boundary between the oil-repellent and flat oleophilic areas was carried out by using scanning electron microscopy (SEM). For this, a PDMS droplet, which has a similar 
surface tension to that of silicone oil, was placed on an adjoining flat oleophilic surface and pneumatically pushed towards the oil-repellent area and then cured. Clear images of the representation of an oil droplet by PDMS at the boundary were obtained as shown in Figure 16. The fluidic resistance faced by the three-phase contact line at the boundary prevents its further advancement.
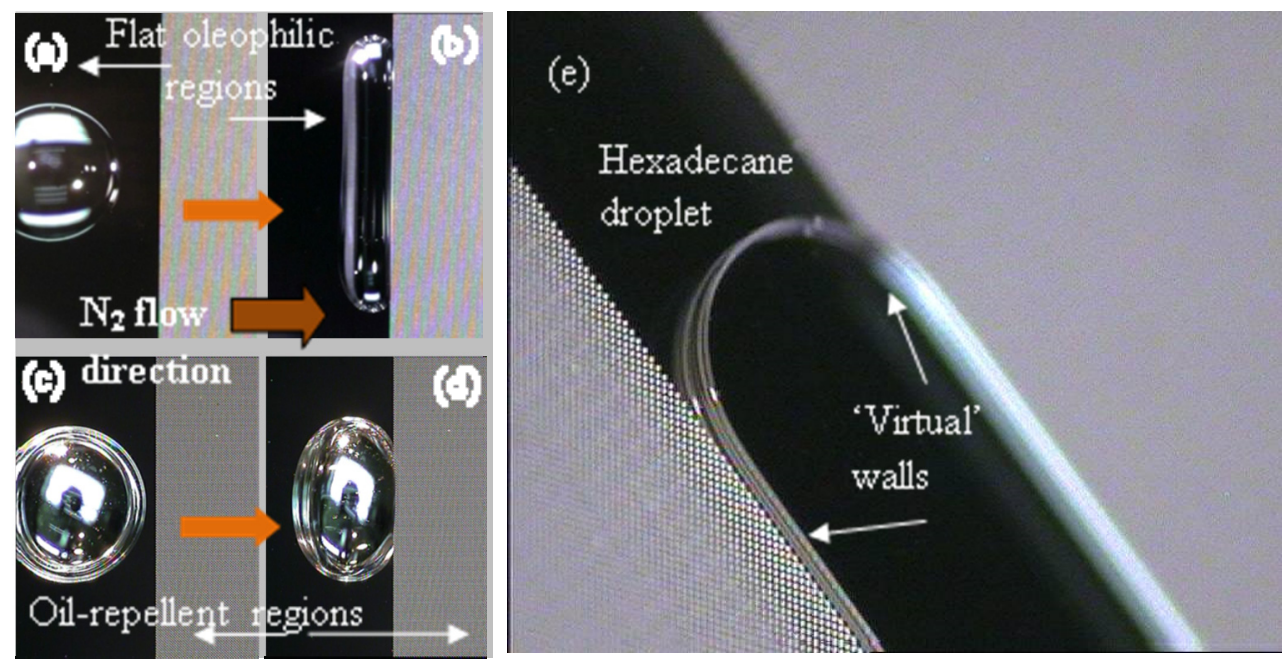

Figure 17. Silicone oil droplet $(a, b)$ and castor oil droplet $(c, d)$ pneumatically actuated $(a \rightarrow b, c \rightarrow d)$ from non-textured towards the textured oil-repellant region. (e) Hexadecane moves along an un-textured path due to the presence of 'virtual walls'.

As can be seen in the above image (Figure 17), a drop of hexadecane selectively wets the flat oleophilic region. The micromachined regions turn out to be too oil-repellent to allow the hexadecane droplet to wet the surface. Even when pushed by a strong pneumatic force, the oil droplet cannot move over the oil-repellent region. Various low surface tension liquids introduced between the micromachined areas can be well confined within the oleophilic pathways as shown in Figure 17e. Because there are no physical walls on the sides of the liquid stream, confinement of low surface tension liquids can be looked upon as confinement by the presence of 'virtual' walls. Virtual walls formed using these oilrepellent surfaces have been exploited for guiding the microfluidic valve on the ejector surface as will be seen in the later part of this chapter. 


\subsection{Fabrication details for oil-repellent surfaces}

Oil-repellent surfaces can be fabricated on a wide variety of substrates. To begin, on a precleaned glass substrate, a $10 \mu \mathrm{m}$ thick SU8 layer is first spin coated on the surface. After curing the SU8 layer, the surface is then metalized for subsequent processing. For this purpose, a thin metallic layer $(0.2 \mu \mathrm{m})$, such as Aluminum (Al), is deposited using RF magnetron sputtering and is used as a template for the surface micromachining of the holes. A patterning step defines the metal template. Thereafter, a reactive Ion etching (RIE) process is used to isotropically etch into the SU8 layer. In this step, an oxygen plasma generated at $250 \mathrm{~W}$ RF power, at a processing pressure of $200 \mathrm{mTorr}$, is used to etch the SU8 within the exposed part of the template for $15 \mathrm{~min}$. The metallic template is then stripped away and the surface is rendered hydrophobic with Cytop in a similar manner as carried out for the ejector surface as described in chapter 2. This completes the fabrication of open channel oil-repellent surfaces as shown in Figure 18(a-d). For supporting low surface tension liquid streams between oil-repellent areas, a transparent top cover slip is then assembled over a $25 \mu \mathrm{m}$ thick spacer and bonded. The final device which is a topcovered microfluidic channel, comprises an oleophilic strip between adjacent oil-repellent areas as shown in Figure 18g. 
(a)

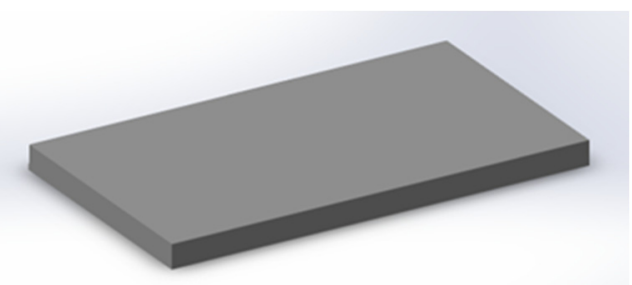

(b)

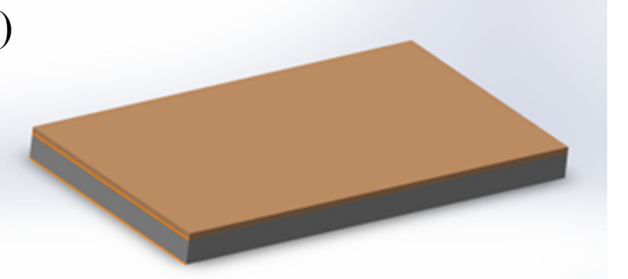

(c)

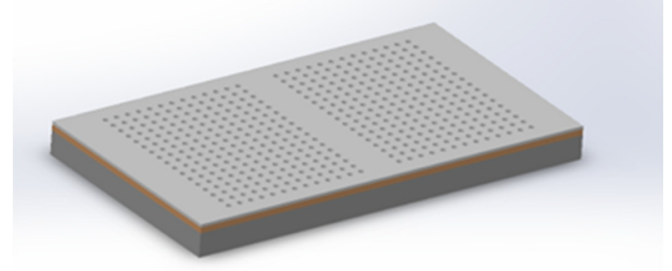

(d)

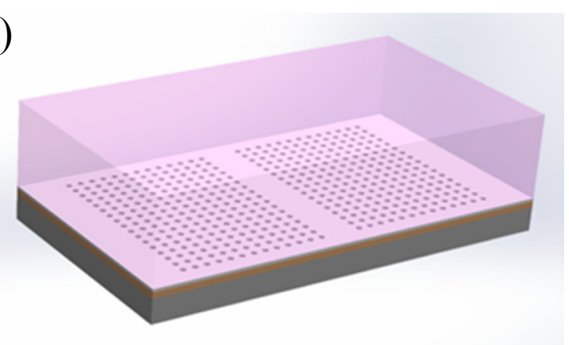

(e)
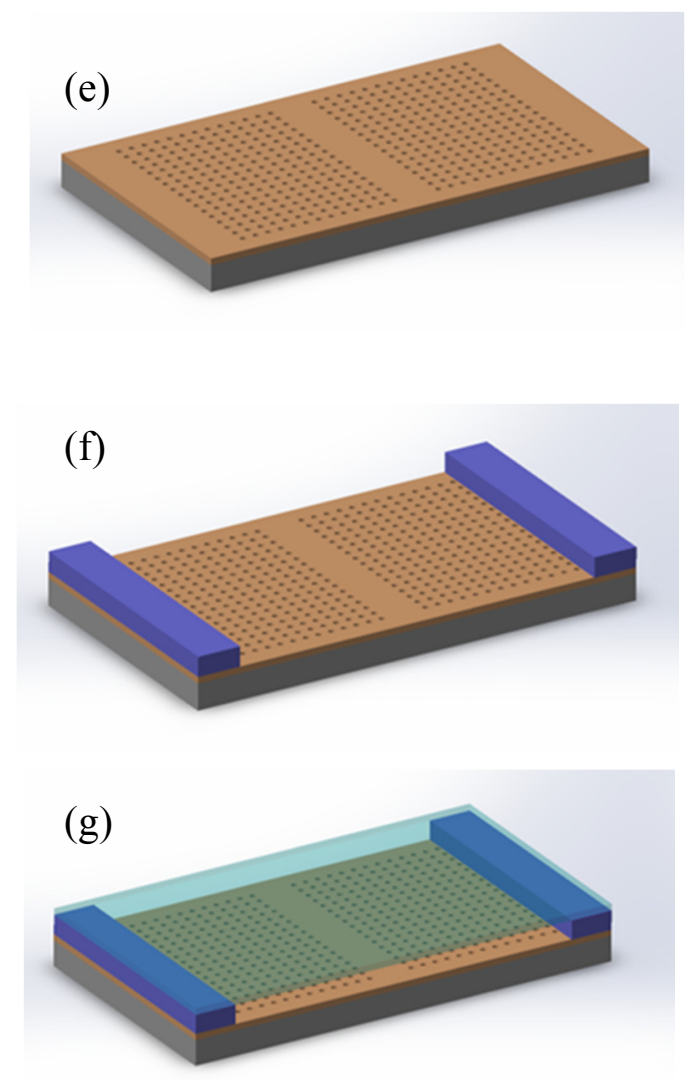

Figure 18. Fabrication steps outlined (a) Piranha cleaning of glass substrate (b) Deposition of SU8 layer (c) Metallization and patterning defining etch mask (d) $\mathrm{O}_{2}$ RIE etch (e) Stripping of metal layer (f) Coating substrate surface and cover-slip with hydrophobic layer and addition of spacers ( $g$ ) Final device assembly. 


\subsection{Study of virtual wall driven capillary flow}

(a)

(b)

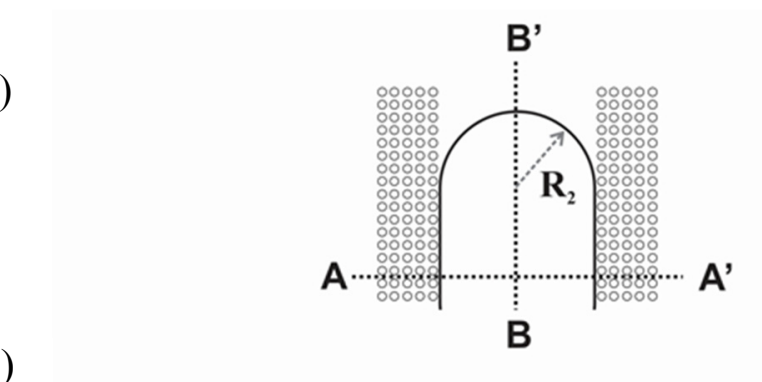

\section{b)}

(c)

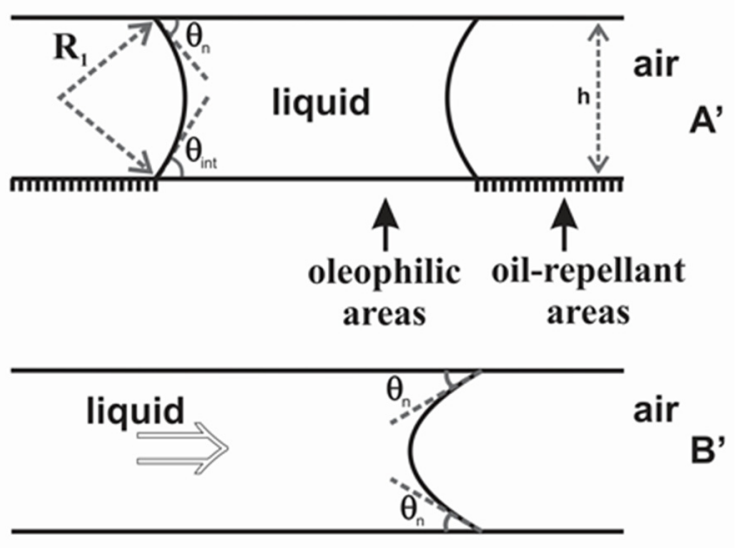

Figure 19. Illustration of important parameters for the virtual walls formed by oil-repellent surfaces in a top-covered microfluidic device such as Young-Laplace radii of curvature and contact angles (a) Top view of a liquid stream confined by virtual walls (b) Cross section view of the liquid stream at $A-A^{\prime}$ (c) Cross section view of the liquid stream at $B$ $B^{\prime}$

An understanding of low surface tension liquid flow propagation along oleophilic pathways is important to further understand the behavior of the microfluidic valve when introduced in the top- covered ejector devices. Figure 19 describes top view (a) and cross section views (b-c) of a liquid stream flowing along the micropath. As soon as a low surface-tension liquid is introduced along the micropath in a top-covered device, two different contact angles can be seen at the oil-repellent / oleophilic boundary and at the advancing front of the liquid. The liquid's internal pressure is released at the advancing front by allowing the liquid to propagate along the oleophilic channel while it increases the contact angle at the oil-repellent / oleophilic boundary and results in a larger radius of curvature as compared to the advancing front. When the contact angle of the free surface of the liquid $\theta_{\text {int }}$ at the 
boundary between the oleophilic and oil repellent areas exceeds the contact angle on the oil-repellent surface (which is $106.6^{\circ}$ for the case of hexadecane), the virtual walls cannot be sustained along the boundary and the TCL will move beyond the next row of micromachined holes. A pressure drop is always present when the liquid-air interface is curved due to surface tension. According to the Young-Laplace equation which is given by $\Delta P=\gamma\left(1 / R_{1}+1 / R_{2}\right)$, where $\Delta P, \gamma, R_{2}$ and $R_{1}$ are the pressure difference, liquid surface tension, and the radii of curvature of the liquid droplet from the top and side views respectively, the pressure on the concave side (Figure 19b and Figure 19c depicting air on the concave side of the liquid-air interface) is always greater than the pressure on the convex side (Figure 19b and Figure 19c depicting liquid on the convex side of the liquidair interface). For the case of an elongated droplet / microfluidic valve (such as the hexadecane droplet shown in Figure 17e), $R_{2}$ for the sides where virtual walls are present is infinite and the Laplace equation is simplified to $\Delta P=\gamma / R_{1}$. This is under the assumption that the TCL at the oleophilic / oil-repellent boundary is a straight line which in reality is actually curved as seen in the SEM image in Figure 16. The many small localized curves of the TCL do not appreciably alter the overall curvature of $R_{l}$ at the boundary. The value $R_{l}$ can be expressed by the equation $R_{1}=h /\left[\cos \theta_{\text {int }}+\cos \theta_{n}\right]$ where $h$ is the channel depth. This expression is obtained from the derivation below for a liquid stream / elongated microfluidic valve constrained between the top cover-slip and the ejector surface resulting in a concave interface where $\theta_{\mathrm{n}}<\pi / 2, \theta_{\mathrm{b}}>\pi / 2$ and $\theta_{\mathrm{n}}+\theta_{\mathrm{b}}<\pi$. 


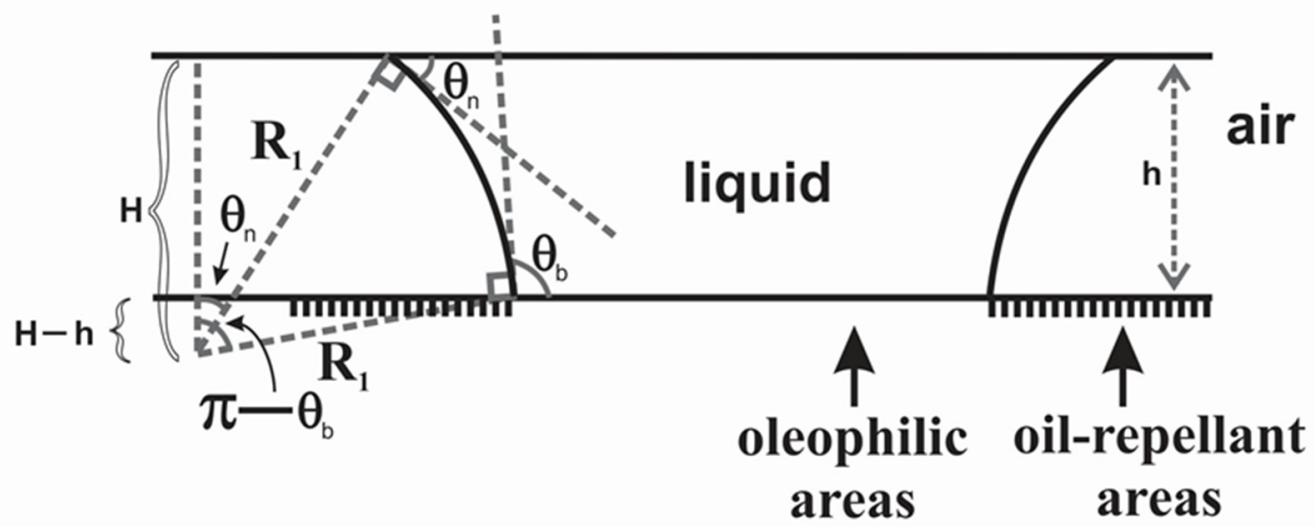

Figure 20. Schematic of the geometry of the cross section of the liquid stream constrained between two parallel plates for the case of a concave interface where $\theta_{n}<\pi / 2, \theta_{b}>\pi / 2$ and $\theta_{n}+\theta_{b}<\pi$.

Here $H$ is the distance from the center of curvature of the liquid-air interface to the top cover plate and $h$ is the channel height which is $25 \mu \mathrm{m}$ in our case. For the case of a stream of hexadecane supported by virtual walls, the liquid stream has a concave profile as shown in Figure 20. Now considering the triangle with sides $H$ - $h$ and hypotenuse $R l$, we have

$\cos \left(\pi-\theta_{b}\right)=-\cos \theta_{b}=(H-h) / R_{1}$

$-R_{1} \cos \theta_{b}=H-h$

Considering the triangle with sides $H$ and hypotenuse $R_{1}$, we have

$$
\begin{aligned}
& \cos \theta_{n}=H / R_{1} \\
& \therefore H=R_{1} \cos \theta_{n}
\end{aligned}
$$

The maximum pressure that the virtual walls supporting the microfluidic valve can sustain is $P_{\max }=\Delta P=\gamma / R_{1}=\gamma\left[\cos \theta_{\text {int }}+\cos \theta_{n}\right] / h$. Theoretical calculations predict the corresponding maximum $P_{\max }$ to be $-348 \mathrm{~N} / \mathrm{m}^{2}$. This value can increase up to $628 \mathrm{~N} / \mathrm{m}^{2}$ 
when both top and bottom surfaces are patterned and aligned. The capillary force generated from the oleophilic surfaces drives liquid forward and produces a concave side-view profile as shown in Figure 19c. with a negative curvature $\left(R_{I}\right)$, whereas the retraction force generated from the oil-repellent virtual walls restrains the liquid from spreading further beyond the boundary and produces a much less concave side-view profile as shown in Figure 19b. We believe this is the underlying mechanism that drives low surface tension liquids such as the microfluidic valve along the oleophilic paths in top-covered devices.

\subsection{Study of defect initiated contact line pinning}

A study of the interaction of the microfluidic valve with a defect-ridden surface was necessary in order to develop microfluidic valve guiding features in top-covered devices. As will be seen in the next chapter, we also implement an open-channel ejector system which exploits a defect-ridden surface for demonstrating a 'Sliding Liquid drop' (SLID) shutter. Furthermore, electric fields in conjunction with surface defects are used to momentarily alter the equilibrium state of the immobilized microfluidic valve on the ejector surface in this latest clog-free ejector prototype. Hence an understanding of surface defects is necessary.

(a) 3-phase contact line on matrix

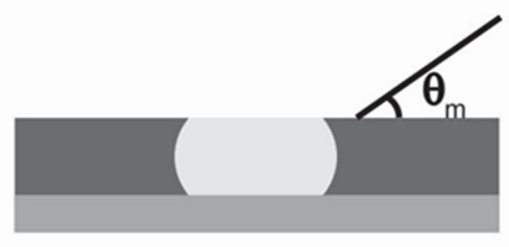

(b) 4-phase contact line

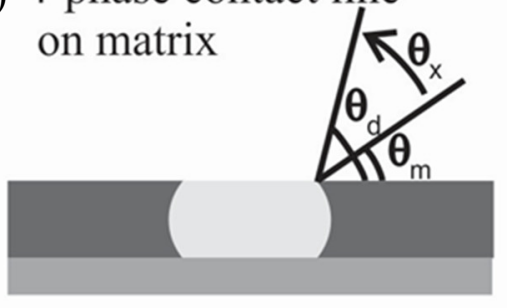

Figure 21. Illustration of (a) the contact angle due to the three phase contact line on a homogenous surface and (b) the defect contact angle at the border of a defect. 
The periodic pattern (hexagonal) of micromachined holes as seen in Figure 16 can be viewed as a surface with a constant defect density having six-fold symmetry. These rows of etched holes represent energy barriers to the moving triple-phase contact line (TCL) as it advances across the surface and is characteristic of a moving elastic medium in a pinning potential [39]. Understanding how a periodic array of defects stops the advancement of the TCL requires a deeper understanding of the interaction of the contact line with a single isotropically etched feature. Cubaud et al [40-42] describe the contact line $\Lambda$ as a set of i) the three-phase contact line (TCL) $\Lambda_{3}$ corresponding to the contact area on a homogenous solid and ii) the four phase contact line $\Lambda_{4}$ corresponding to the border of the two homogenous solids (the flat solid surface and the defect surface - represented by a virtual surface and having a finite contact angle) when the size of the defects $d$ (in this case $d=20$ $\mu \mathrm{m})$ is larger in width than that of the three phase contact area $\delta$ approximately equal to a few nanometers [43]. This is analytically described by $\Lambda=g(t) \Lambda_{3}+(1-g(t)) \Lambda_{4}$ where $g(t)$ is a function of time and surface geometry. For a quasi-static motion of $\Lambda_{3}$ with a small capillary number $(\mathrm{Ca}<<1)$, although the associated contact angle is considered unique, the contact angle $\theta_{x}$ along the static four phase contact line $\Lambda_{4}$ varies between the contact angle $\theta_{m}$ on a flat homogenous surface and the defect contact angle $\theta_{d}$. As the liquid travels across the surface, the presence of a defect along its path deforms the contact line $\Lambda$. If the defect and/or the wettability contrast is small enough (weak defect), the contact line recovers its unperturbed position after passing the defect. On the contrary, if the defect and/or the wettability contrast is too large (strong defect), the defect traps the line and stays uncovered by the liquid layer. 
The wettability contrast is defined as the difference in spreading coefficients between the defects and the flat homogenous surface which is given by $\Delta S=\gamma\left(\cos \theta_{d}-\cos \theta_{m}\right)[41]$. In the present case, $\theta_{d}=180^{\circ}$ and $\theta_{m}=53^{\circ}$ for air and hexadecane respectively giving $\Delta \mathrm{S}$ $=24.4 \mathrm{mN} / \mathrm{m}$. The defect strength $f$ is a relevant dimensionless parameter used to describe the influence of the wettability defect and is given by $f=\Delta S d / \gamma h_{m}$ where $h_{m}$ is defined as the drop thickness [41]. The defect strength can be understood as the ratio between the pinning force of the defect and the elastic restoring force of the TCL due to surface tension. Now, surface tension acts in a way to minimize the free surface area, so the wetted surface of the drop is as compact as possible. If the energy barriers of the rows are strong enough, they trap the edge of the drop as seen in Figure 16. When the contact line is completely trapped by these energy barriers on a surface with a constant defect density, the thickness of the drop increases so the defect force decreases. Hydrostatic pressure increases with increasing drop thickness and eventually covers the first few rows of defects as seen in Figure 22 below.

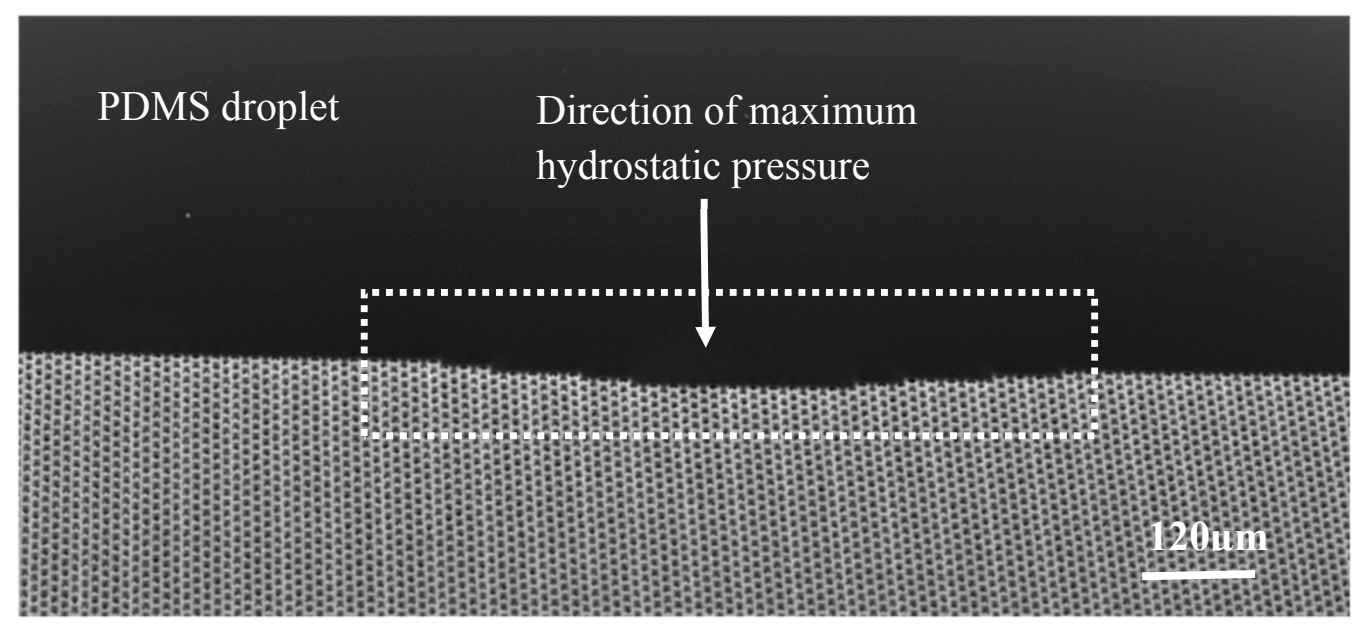

Figure 22. SEM image of a PDMS droplet at the boundary between an oleophilic and oil repellent-area. 
A hexagonal defect pattern was implemented for increased robustness while designing the oil-repellent surface. This gives the surface a six-fold symmetry to the approaching TCL. The probability of the TCL de-pinning at the boundary between the flat oleophilic and the oil-repellent areas is the same at each of the defects along the boundary. A defect dimension of $20 \mu \mathrm{m}$ was chosen based on empirical data while designing superoleophobic surfaces. It was observed that the mushroom microstructures used in superoleophobic surfaces with these dimensions and similar periodicity could stably trap pockets of air under the liquid droplet. When the defect size is made larger than $20 \mu \mathrm{m}$ such as $30-40 \mu \mathrm{m}$, the contact angle of a hexadecane droplet exhibited a much smaller value similar to that on a flat surface $\sim 53^{\circ}$. This proves that the pockets of air could no longer be sustained in the micromachined defects. When this happens, the wettability contrast of the surface becomes very small and the surface loses its oil-repellency. On the other hand, making the defect dimensions smaller than $20 \mu \mathrm{m}$ would increase the stability of the trapped air pockets but would decrease the defect strength $f$ for a given defect periodicity. However, the decreased defect strength could be compensated by reducing the defect separation thereby increasing the defect density. Optimizing the surface for maximizing the oil repellency cannot be done with a single parameter alone and requires the optimization of more than one parameter at a time.

\subsection{Characterization of oil-repellent surfaces using contact angle measurements}

In order to characterize the oil-repellent surfaces better, the contact angles of various test liquids with different surface tensions were measured on the newly developed oil-repellent surfaces and a comparison was made between the measured and the apparent contact angles 
using the Cassie-Baxter relationship which is given by $\cos \theta_{Y}^{C B}=r_{\phi} \phi_{S} \cos \theta_{Y}+\phi_{S}-1$. In this expression $\theta_{Y}^{C B}$ is the apparent equilibrium contact angle in the Cassie-Baxter state, $r_{\phi}$ is the roughness of wetted solid, defined as the actual wetted surface area divided by its projected surface area; $\phi_{S}$ is the solid fraction, defined as the projected wetted surface area divided by the nominal surface area, $\theta_{Y}$ is the intrinsic contact angle on smooth and flat surfaces, given by Young's equation $\cos \theta_{Y}=\left(\gamma_{S V}-\gamma_{S L}\right) / \gamma_{L V}$ where $\gamma$ is the surface tension, and subscripts $\mathrm{S}, \mathrm{V}$, and $\mathrm{L}$ represent solid, vapor and liquid, respectively. For such a surface, $r_{\phi}$ was assumed to be 1 with the assumption that the wetted area was equal to the projected area and $\phi_{S}$ was calculated to be 0.6 from the initial design and 0.488 through image analysis of the SEM images for the surface. The graph in Figure 23 shows that the apparent contact angles using the Cassie-Baxter relationship are far different from the measured values. The extent of liquid penetration into the etched holes cannot be easily determined and this could explain the large discrepancies in the observed and the predicted contact angles. The filling up of the etched holes would lead to the displacement of air pockets. During measurements, no such displaced air pockets were observed with the exception of one of the liquids - dodecane $\left(25.35 \mathrm{~mJ} / \mathrm{cm}^{2}\right)$. Such discrepancies between the measured and the predicted contact angles were pointed out by many groups and many modifications have been proposed to such a model [30,31]. Since re-entrant curvatures are present within the holes on the oil-repellent surface, it is unclear if air pockets are trapped underneath the liquid droplet when the droplet is placed on the surface. Further studies would need to be done to confirm this phenomenon. 


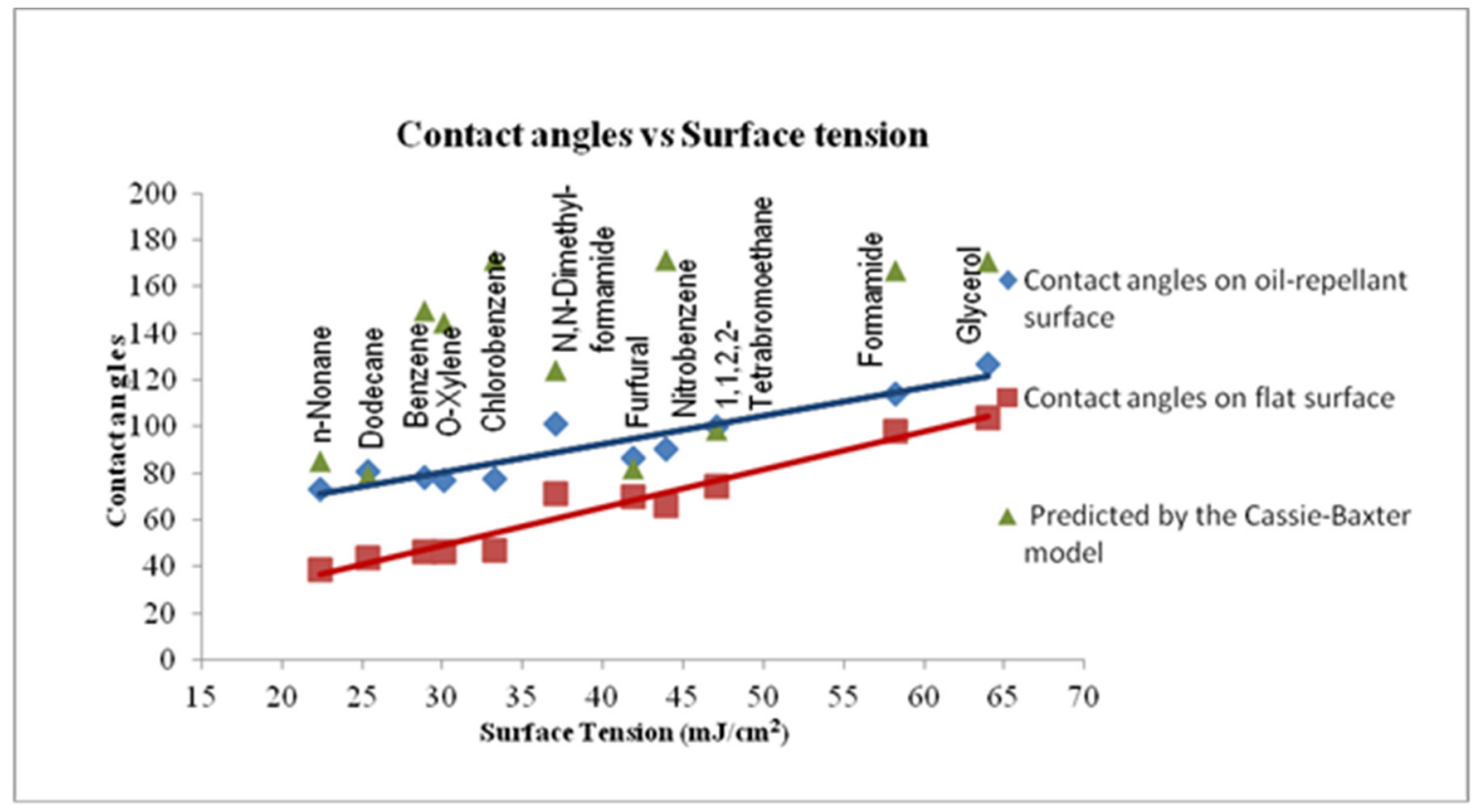

Figure 23. Graph of observed contact angles vs surface tension values of various low surface tension microfluidic valve materials tested on our oil-repellent surface and flat surfaces.

\subsection{Guiding of the liquid-core microfluidic valve on ejector surface using oil-repellent surfaces}

Guiding of the integrated microfluidic liquid valve onto and off the nozzle along a predefined boundary was the original motivation behind the development of the oilrepellent surfaces. Such a functionality is a desirable feature of the microfluidic ejector. The control of surface wettability for manipulating various microfluids has been widely adapted in many microfluidic applications. Although manipulating and controlling aqueous solutions are relatively easy, it is very difficult to prevent spreading of non-aqueous low surface tension liquids (oils such as silicone oil, castor oil etc. used as the microfluidic valve material) onto non-desired regions. In addition, superoleophobic surfaces inside microfluidic channels have not been broadly explored yet. For this purpose, a microfluidic channel with virtual walls has been designed for the LDEP guiding of the microfluidic 
valve across the ejector surface using the newly developed oil-repellent surface. Initially, superoleophobic surfaces were evaluated for their lateral guiding capability to the microfluidic valve across the ejector surface. However, during subsequent tests, it was realized that superoleophobic surfaces were not effective in guiding microfluidic valve liquids across surfaces. Hence, plasma-assisted patterning of surfaces that constitute oilrepellent surfaces were used to precisely guide the microfluidic valve along predefined paths. This is the first time that guiding of low surface tension liquids, such as the microfluidic valve liquid, along predefined paths has been demonstrated inside closedchannel devices. As can be seen in Figure 24b, a droplet of silicone oil is guided using LDEP along the predefined path bounded by the oil-repellent surface. Unlike earlier, the microfluidic valve no longer has a circular shape but is elongated due to liquid confinement by the virtual walls.

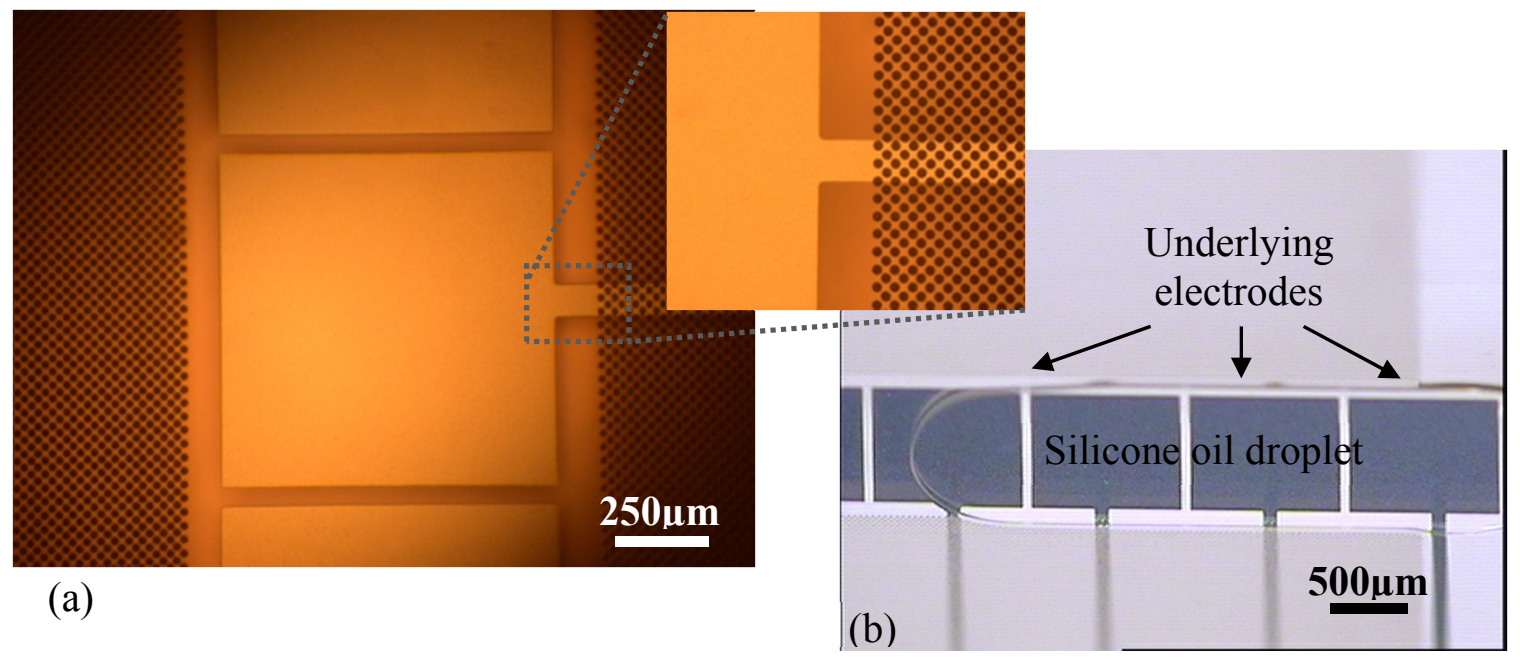

Figure 24. (a) Optical microscope image of the top-view of the underlying electrodes between the oil-repellant areas. (b) Silicone oil droplet confined between the oil-repellant surfaces taking on an elongated shape due to confinement by virtual walls. All of the electrodes are un-actuated. The underlying electrodes that aid in the actuation of confined liquids via dielectrophoretic forces can clearly be seen. 


\subsection{Fabrication and working mechanism of guiding features on ejector surface}

Fabrication of the ejector surface integrated with microfluidic valve guiding functionality begins with building upon the conventional ejector surface which is described in detail in the earlier part of this dissertation in chapter 2. On the pre-fabricated ejector surface, a 10 $\mu \mathrm{m}$ thick SU8 layer is first spin coated on the surface onto which the microfluidic valve guiding structures are built. After curing the SU8 layer, the surface is then metalized for subsequent processing. For this purpose, a thin metallic layer $(0.2 \mu \mathrm{m}$ thick $)$ is deposited using RF magnetron sputtering, and which is used as a template for the surface micromachining of the holes. A reactive Ion etching (RIE) step, in an oxygen environment, is used to isotropically etch into the SU8 layer. The metallic template is then stripped away and the surface is rendered hydrophobic with Cytop in a similar fashion as carried out for the ejector surface. The top cover-slip is then assembled over a $25 \mu \mathrm{m}$ thick spacer and bonded. The final device, which is a top-covered microfluidic channel, comprises the oilrepellent features micromachined into the $10 \mu \mathrm{m}$ thick SU8 layer above the underlying driving electrodes and bounded by physical walls only by the top and bottom surfaces. Since the electrodes are not in direct contact with the liquids, we believe that dielectric as well as conducting liquids can be driven along the virtual microfluidic channel using this fabrication scheme. 


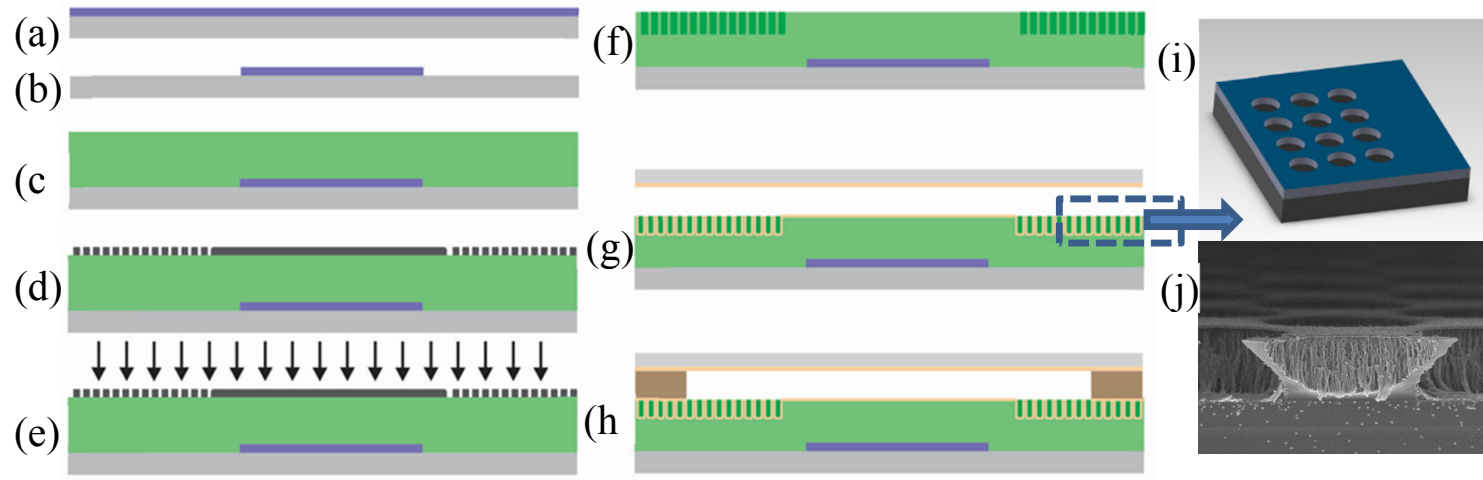

Figure 25. Fabrication steps outlined (a) Metallization (b) Patterning of metal layer to define electrodes (c) SU8 layer (d) Metallization and patterning defining textures (e) $\mathrm{O}_{2}$ RIE etch (f) Stripping of metal layer (g) Coating substrate surface and coverslip with hydrophobic layer (h) Final device assembly via spacers. (i) Schematic of the micromachined features on the surface and (j) SEM image of the cross -section view of one of the micro-machined features comprising the oil-repellant surface.

Activation of the electrodes with a DC voltage source and consequent generation of dielectrophoretic forces in the confined oil droplet (microfluidic valve) cause it to move along the oleophilic micropath as shown in the Figure 26 below. Furthermore, the motion of the confined low surface tension dielectric liquid film is governed by liquid dielectrophoresis (LDEP). Due to the polarization of the dielectric fluid in response to the external electric field, the dielectrophoretic force is exerted on the liquid. These dielectrophoretic body forces generated by the application of the electric field on the droplet dominantly contribute to the motion of the dielectric liquid in the microfluidic channel confined by virtual sidewalls. The straight liquid-air interfaces supported by virtual walls can clearly be seen in Figure 24b. 


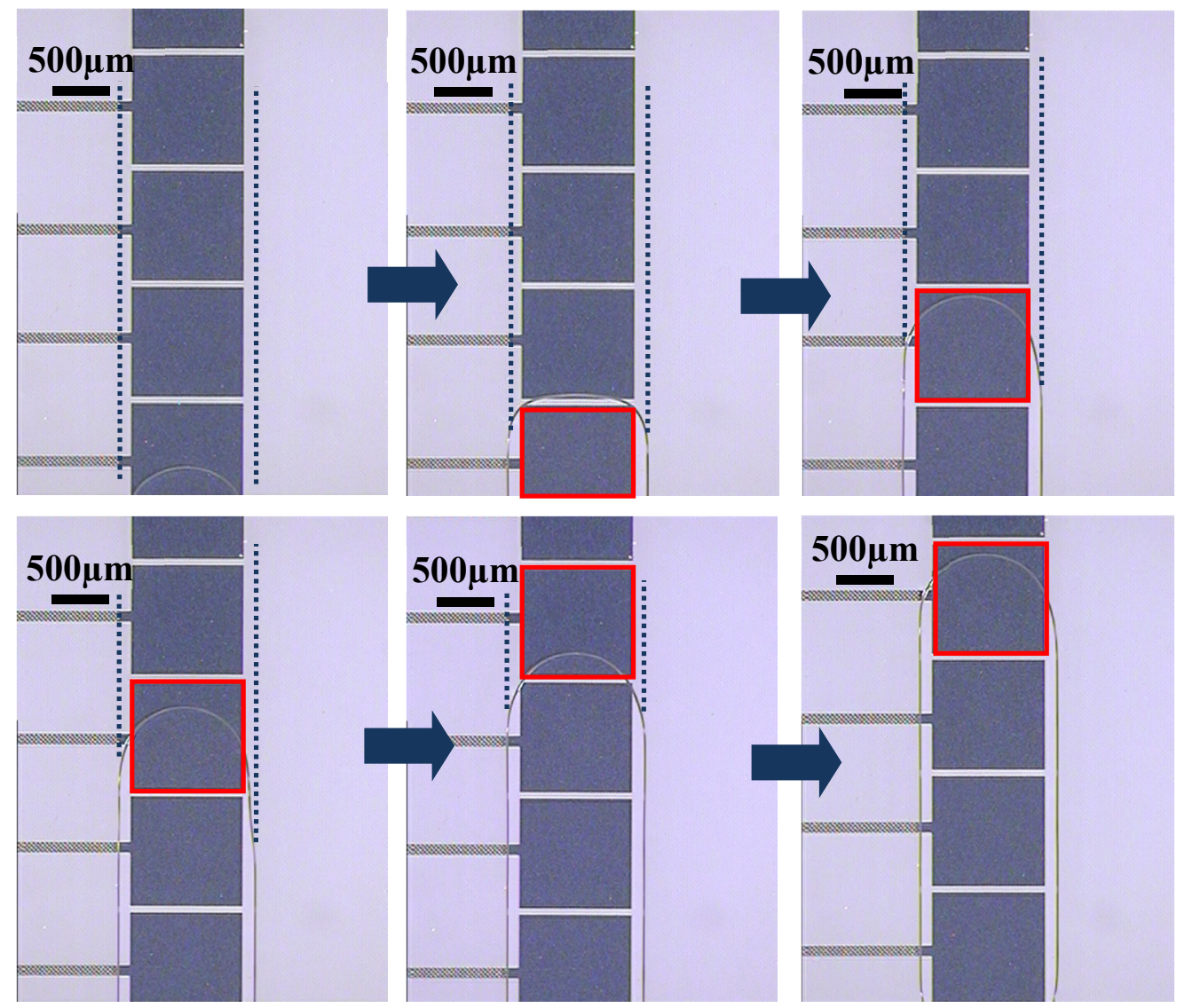

Figure 26. Droplet (silicone oil) actuated across the desired path using liquid Dielectrophoresis (L-DEP) by actuating one electrode at a time. Dashed lines have been used to depict the position where virtual walls will be formed as the droplet progresses.

Compared to viscous fluids such as silicone $(50 \mathrm{cSt})$ and castor $(985 \mathrm{cSt})$ oil, it was observed that less viscous fluids such as hexadecane and Fluorinert FC-70 flow more easily along paths confined by micromachined oil-repellent surfaces. The liquid-air interfaces along the sides of the microfluidic valve look clearly straight as seen in Figure 26. Furthermore, the results from the actuation of various test liquids are summarized in Table V. 
TABLE V. Actuation voltages with and without the use of micro textured guiding of various low surface tension liquids

\begin{tabular}{|l|c|c|c|c|}
\hline Test liquids & $\begin{array}{c}\text { Actuation } \\
\text { voltage without } \\
\text { micro textures }\end{array}$ & $\begin{array}{c}\text { Actuation voltage } \\
\text { with micro textures }\end{array}$ & $\begin{array}{c}\text { Di- } \\
\text { electric } \\
\text { constant }\end{array}$ & $\begin{array}{c}\text { Surface } \\
\text { tension (mJ/ } \\
\left.\mathbf{c m}^{\mathbf{2}}\right)\end{array}$ \\
\hline $\begin{array}{l}\text { Silicone oil } \\
(50 \mathrm{cSt})\end{array}$ & $310 \mathrm{~V}$ & $325 \mathrm{~V}$ & 2.75 & 20.3 \\
\hline $\begin{array}{l}\text { Castor oil } \\
(985 \mathrm{cSt})\end{array}$ & $316 \mathrm{~V}$ & $450 \mathrm{~V}$ & 4.7 & 39 \\
\hline $\begin{array}{l}\text { Hexadecane } \\
(3.02 \mathrm{cSt})\end{array}$ & $\sim 400 \mathrm{~V}$ & $420 \mathrm{~V}$ & 2.05 & 27.4 \\
\hline FC-70 $(24 \mathrm{cSt})$ & $188 \mathrm{~V}$ & $210 \mathrm{~V}$ & 1.98 & 18 \\
\hline
\end{tabular}

In summary, micromachined features were implemented on the ejector surface for guiding the microfluidic liquid valve along the surface. The microfluidic liquid valve was characterized by straight sidewalls while guided by the micromachined features (also termed as oil-repellent features) during actuation. Furthermore, the electrical actuation of various test liquids along oleophilic paths bounded by oil-repellent features has shown a lot of success. This technique can be applied to many microfluidic devices dealing with various low surface tension liquids such as the microfluidic ejector. Since these micromachined features could be used to prevent the spread of low surface tension liquids, they were categorized as oil-repellent features. 


\section{Chapter 4}

\section{LOW VOLTAGE DRIVING OF MICROFLUIDIC VALVE AND DEVELOPMENT OF SLIPPERY SURFACES}

\subsection{Development of hydrophobic / oleophobic surfaces for reduction in microfluidic valve driving voltage}

Friction in fluids manifests itself by the phenomena of drag - the force required to move an object through a fluid or a fluid through a device [44]. There are currently few demonstrated methods for significantly reducing drag using superhydrophobic surfaces in devices where the fluid flow is laminar and not turbulent [44, 45]. By reducing the microfluidic liquid valve drag along the ejector surface, the driving voltage could be reduced. In order to reduce the microfluidic valve driving voltage further, we initially explored superhydrophobic and superoleophobic surfaces on the ejector surface for this purpose.

Superoleophobic surfaces were developed using a bulk micromachining approach that would eventually enable us to create such surfaces onto the bio-ejector surface. Since most of the bio-fluids to be tested in the bio-ejector have very low surface tensions like most hydrocarbons, superhydrophobic coatings do not help much and in fact worsen the situation by causing low surface tension liquids to easily spread and form a thin layer which could affect the performance of the microfluidic valve. We initially conjectured that the creation of a superoleophobic surface would help us to actuate the liquid-core microfluidic valve onto and off the ejector nozzle with a lower electrical potential and also prevent any residual bio-fluids from being left behind. Furthermore, such a surface would possess superior self-cleaning properties to most oily liquids surpassing the self-cleaning properties 
of the leaves of the 'Nelumbo nucifera' plant commonly known as the lotus. A 'top-down' approach for the creation of a superoleophobic surface had already been successfully fabricated and tested. Contact angles $>140^{\circ}$ had been achieved with hexadecane with the droplets being almost spherical when deposited on the surfaces. In a similar 'bottom-up' approach that was being developed, 3D microstructures were to be fabricated which would be similar to the microstructures fabricated in the top-down approach. Since the top-down approach is much simpler from the fabrication aspect than the bottom-up approach, all the parameters (structure spacing and structure diameters) required to make robust superoleophobic surfaces were to be derived from these surfaces and then applied to the structures in the bottom up approach.
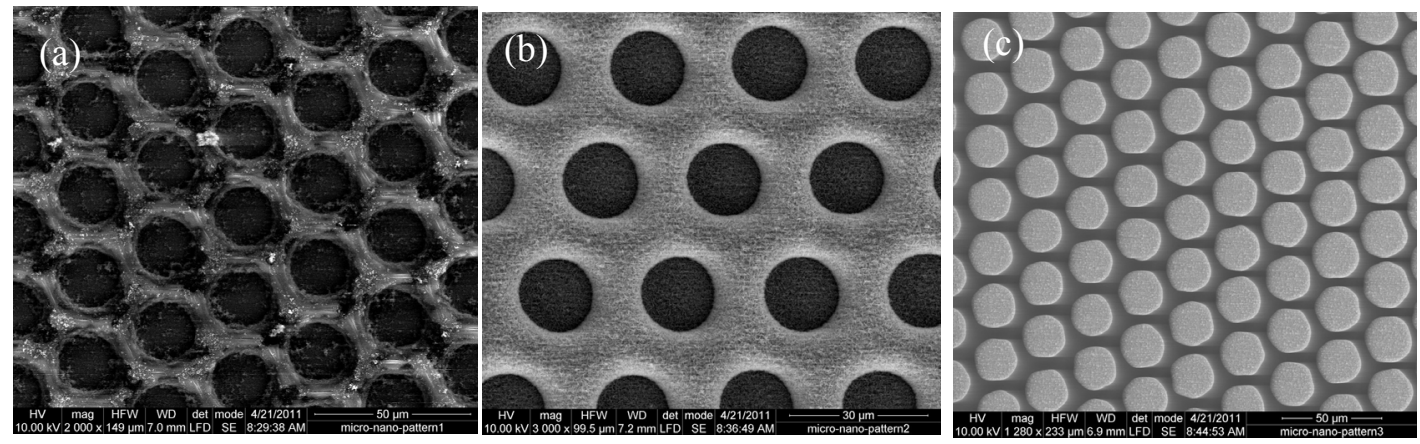

Figure 27. Scanning electron microscopy (SEM) images of: - (a) Low surface energy nanoparticle coatings on cured photoresist (PR) microstructures (b). Zinc Oxide nanorods grown on similar PR microstructures (c) Mushroom-like microstructures formed using electroless Nickel $\sim 10$ um tall.

Several techniques were implemented in developing superoleophobic surfaces using bottom up approaches. In one approach, hierarchical structures composed of micro and nanostructures were built using two different methods. In the first method, nanoparticles were spin coated directly onto patterned microstructures and then coated with a very thin hydrophobic layer as seen in Figure 27a. In another similar method, nanorods were grown 
directly onto the microstructures using liquid precursors as seen in the SEM images in Figure 27b. Still in another approach, micro-pillars were fabricated using electroless nickel plating to form $10 \mu \mathrm{m}$ tall pillars which corresponded to the thickness of the photoresist mold. From this point onward, the pillars were overgrown to form mushroom-like structures. It has recently been discovered that such structures could cause aqueous [46] as well as low surface tension liquids such as dodecane, hexadecane, etc. to remain in a Cassie state with pockets of air trapped below the droplet [28, 29, 46, 47]. Such microstructures with overhanging features are very crucial in the creation of superoleophobic surfaces as will be seen in the later part of this chapter.

Despite all efforts in developing superoleophobic surfaces using different approaches on the ejector surface, the effective lowering of the microfluidic valve driving voltage could not be demonstrated. Some of the biggest problems encountered were the microfluidic valve transitioning from the Cassie state to the Wenzel state and getting pinned onto the ejector surface. Once this occurred, driving the microfluidic valve even with high driving voltages was not possible. However during this time, a new surface type was discoveredthe 'slippery' surface. This new surface type was used to impart additional functionality when implemented on the ejector surface. Furthermore, these new surface types were extensively explored and have been reported in the later part of this chapter.

\subsection{Slippery surfaces for open channel microfluidic valve actuation}

Micro and nano-engineered surfaces using bottom-up, top-down and self-assembly have gained widespread importance in the last decade or so. Furthermore, synthesizing nonwetting surfaces such as omniphobic surfaces that can repel aqueous / non-aqueous liquids, biological fluids as well as oily liquids - liquids spanning a large gamut of surface tensions 
- has also been demonstrated. However, designing surfaces that can repel as well as manipulate liquid droplets on their surface has been a great challenge. Despite great advances in synthesizing surfaces that are ultra-repellent to liquids, there are still challenges that need to be addressed. For example, these surfaces cannot be used to manipulate liquid droplets very effectively using electrical actuation schemes. They rely on gravity to help liquid droplets roll or slide off. Furthermore, most of these surfaces rely on air cushioning to remain stable which can destabilize under the influence of electric fields. Recently, efforts have been focused on replacing the 'air cushioning' with lubricant liquids thus improving stability. However there has been no report on the manipulation of liquid droplets on these surfaces using other schemes. In this work we have synthesized a liquid infused non-wetting surface with the addition of a mobility function. Liquid dielectrophresis (LDEP) has been used as the primary actuation mechanism for the movement of liquids on these slippery surfaces. This surface has been shown to repel different liquids such as hydrocarbons, crude oil and biological fluids such as blood, milk, water etc. naturally, as well as electrically in some cases. Furthermore, this surface has immense potential for surface microfluidic systems that are used for the transport of liquid droplets such as the microfluidic valve across surfaces.

\subsection{Motivation for developing slippery surfaces}

Mimicking the lotus leaf was one of the first attempts in developing artificial textured surfaces inspired from nature [17-19]. It was from this plant that researchers understood the mechanisms that govern the non-wetting properties of natural surfaces. It has been well established that the surface properties of natural surfaces are governed by the complex interplay between morphology and physical/chemical properties [48, 49]. Furthermore the 
features governing the surface properties rely on a hierarchical scheme of dimensions ranging from the microscale to the nanoscale $[17,50]$. Such hierarchical structures are extremely widespread in nature. Roughness at a hierarchical scale - at the micro and nano levels - was the key in developing robust superhydrophobic surfaces. It was later deduced that the presence of air pockets which forms composite solid-air interfaces was responsible for the superhydrophobic behavior of these surfaces $[48,49,51,52]$. In almost all of the artificial surfaces created for non-wetting applications with aqueous solutions, the leaves of the lotus plant proved to be the paradigm for naturally inspired bio-mimicked surfaces. However, natural surfaces such as plant leaves of certain floral species including the lotus have evolved in nature up to the point where the intended requirement had been met. And thus natural surfaces too have limitations outside of their denizens. For example, impinging rain droplets can break apart upon impact and penetrate the textured surfaces of lotus leaves rendering them no longer superhydrophobic. Also these surfaces are prone to localized defects that can cause pinning of the liquid droplets preventing them from rolling off easily. Furthermore, the air cushioning is not sufficient enough to prevent wetting when low surface tension liquids such as most hydrocarbons and organic liquids are applied. For example, a lotus plant would not remain un-wetted in an oil-slick affected region. This becomes a problem for practical implementation of bio-mimicked surfaces. It would be safe to assume that the work of evolution that occurred millions of years ago could not account for the exploits induced by humans in nature. Hence bio-mimicking might sometimes have limitations for the intended application. Despite all these drawbacks, scientists have tried to thoroughly understand the role of the waxy coatings along with the roughness at different hierarchical scales that make these surfaces - lotus leaves - non- 
wettable $[17,18,50]$. This was done so as to further improve the surface characteristics of artificially created surfaces in order to extend their functionality and make them more durable. One successful outcome of such efforts has been the development of superoleophobic surfaces which has been reported by several groups [28, 47]. Superoleophobic surfaces are characterized by additional re-entrant features which distinguish them from their superhydrophobic counterparts. Thus by introducing additional features to existing bio-inspired superhydrophobic structures, the air pockets that make the surface non-wettable could remain stable against low surface tension liquids making the surface more durable.

\subsection{Learning from nature: the Nepenthes pitcher plant}

Another plant surface that has bio-inspired researchers has been the nepenthes pitcher plant [53-56]. The pitcher of this plant uses textured surfaces to keep a certain amount of liquid 'locked' into its surface and then uses such a liquid to form a repelling surface to trap other liquids and foreign particulates - in this case small insects and even small animals - which are eventually ingested with the help of digestive enzymes. The slippery inner surfaces of the pitchers, which can be waxy or highly wettable, have so far been considered as the key trapping features. In Nepenthes pitcher plants, prey capture and retention is mainly thought to be fulfilled by the slippery waxy layer which covers the upper inner side of the pitcher in most species. In other species, the surface energy of the micro-textured roughness and that of the 'locked-in' liquid - most often rain or condensed water - are well matched and as a result, the liquid forms a continuous overlying film. In these plant surfaces, this liquid

film forms a slippery surface that causes miniscule objects such as insects as well as liquid droplets to slip on such surfaces thus being trapped within the pitcher $[1,18,57]$. 


\subsection{Bio-mimicked surfaces with imbibed functionality}

Inspired by the Nepenthes pitcher plants, we have attempted to engineer a surface that could extend the non-wetting ability seen in natural as well as artificially mimicked / synthesized surfaces to a wider range of liquid surface tensions. The addition of a mobile functionality to manipulate liquids on these surfaces was also carried out. While the nonwetting behavior of these surfaces was largely bio-inspired from the pitcher interior, the mobile functionality has been an imparted characteristic. A surface in a wetted state by a liquid (lubricating liquid) at all times will not to be wetted by a secondary liquid immiscible to the lubricating liquid. In other words, a secondary liquid placed on such a pre-wetted surface would not be able to wet the underlying substrate as the surface is already in the wetted state by the lubricating liquid. Such types of surfaces have been synthesized by several groups $[53,55,56,58-60]$. However, motion control of the secondary liquid has never been demonstrated. Here we demonstrate and show how the motion of the secondary liquid droplets on these surfaces can be electrically controlled.

Recent studies have reported that the non-wetting ability can be achieved by replacing the pockets of air with a lubricating liquid $[53,55,56,59,60]$. In the surfaces that we have engineered, a lubricant is uniformly spread by capillary forces arising from the micro / nano textures and allows a secondary immiscible droplet above it to easily glide across the surface while exhibiting extremely low contact angle hysteresis. As shown in previous studies, these surfaces can also self-heal by capillary wicking upon damage and continue to repel liquids. 
For easy motion control of the secondary liquid, our design of these surfaces has been conceptualized from the surface structures seen in certain superoleophobic surfaces along with the wetting mechanisms seen in the Nepenthes pitcher plants. Unlike generic superoleophobic surfaces composed of solid and air fractions, our surface provides liquidpassivated solid surfaces and air fractions.
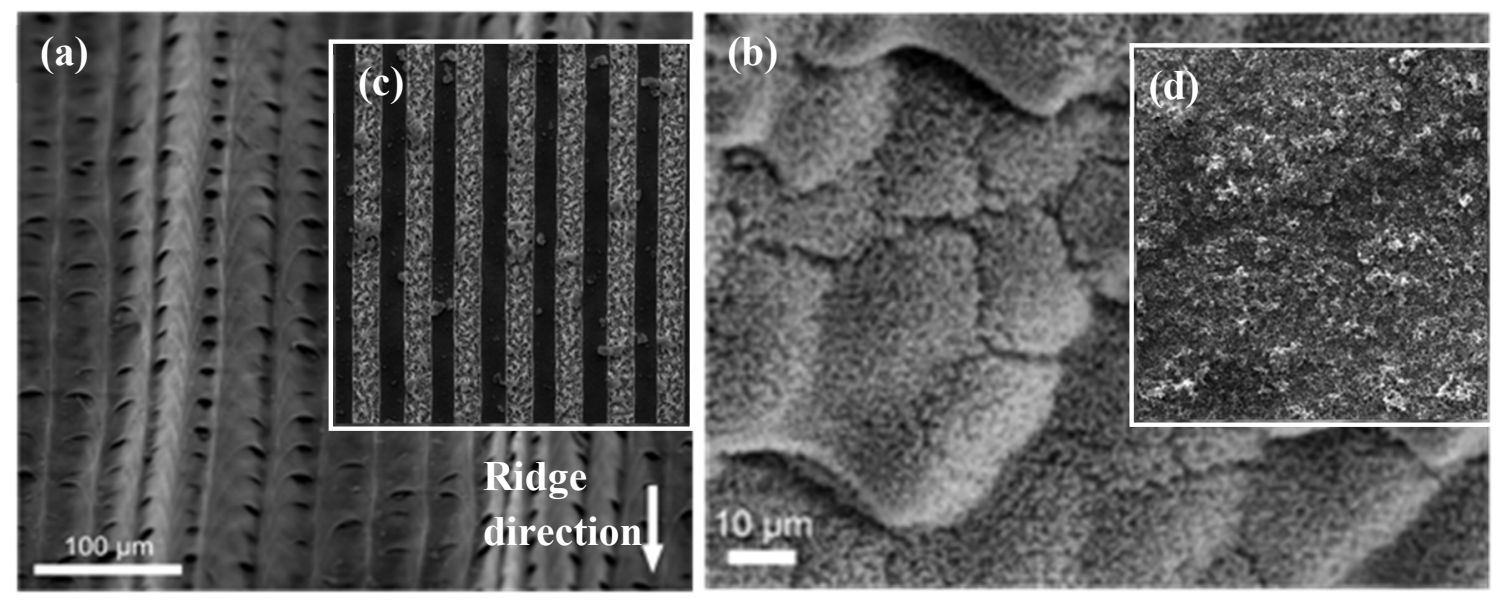

Figure 28. Bio-inspired electrode design and surface morphology. (a) Peristome surface of Nepenthes 'bicalcarata' exhibiting radial ridges directed towards the inside of the pitcher. COPYRIGHT 2004 National Academy of sciences, U.S.A [1] (b) Waxy inner pitcher wall at the height of the inner peristome margin. COPYRIGHT 2004 National Academy of sciences, U.S.A [1] (c) Electrodes microfabricated onto a surface with roughened inter electrode spacing in an attempt to mimic the ridges seen inside the pitcher interior. (d) Nano-coated surfaces designed to mimic the waxy pitcher interiors as shown

In the pitcher, the ridges are directed towards the center of the pitcher interior and as a result liquid droplets easily slide along these ridges towards the interior. Although a similar phenomenon is seen on our surfaces in the absence of an electric field, droplet maneuverability is independent of ridge direction and is governed by the underlying electrode positions when actuated. Slippery surfaces composed of features having multi fold symmetry such as a pillar structures or surfaces composed of homogenous porous/roughened structures would be ideal. However the electrodes which are necessary for electrical actuation, introduce surface anisotropy similar to the Nepenthes pitcher. But 
unlike the pitcher where gravity facilitates movement along the ridges, these surfaces are effective for droplet manipulation in any intended direction by the applied electric fields. For this, a groove-structured design was necessary to employ efficient electrical actuation of liquid droplets on the slippery surfaces via liquid dielectrophoresis (LDEP). Initial application of the lubricating liquid to uniformly wet and coat the micro-grooved surface was a concern due to the anisotropic morphology. However, drainage of liquids from micro-grooved surfaces $[61,62]$ as well as the associated anisotropic wetting behavior of liquid droplets on such type of surfaces [63-65] of different ridge dimensions have been well studied by many researchers. Understanding this anisotropic wetting phenomenon helps during the application of the lubricating liquid. Furthermore, one of the many advantages of using micro-grooved structures for certain applications as compared to other structures such as micro pillars is the improved sliding properties of the secondary liquid droplets such as water in the direction of the grooves. Secondary liquid droplets, especially water droplets, deposited on the grooved surfaces exhibit an anisotropic sliding behavior due to this reason in the absence of the lubricating liquid. This behavior is similar to the anisotropic wetting tendency seen in naturally grooved surfaces such as rice plant leaves and butterfly wings $[66,67]$. In those surface types, it was observed that liquid droplets drained more effectively in the direction of the grooves due to the continuous nature of the contact line on the ridges. Even in the absence of the lubricating liquid, the improved drainage behavior of low surface tension liquids such as oils on nano textured microgrooved surfaces over non-grooved flat surfaces was evident. In a simple test, droplets of silicone oil were placed onto the grooved and non-grooved (flat) regions of the slippery sample in the absence of the lubricating liquid and were simultaneously flushed away from 
the two regions using a nitrogen stream. As can be seen in the images (Figure 29a-d), it was observed that oil drained more effectively from the micro-grooved surface as compared to the non-grooved surface where oil residue can be seen pinned to the nanotextured surface.

\section{Nitrogen \\ direction}

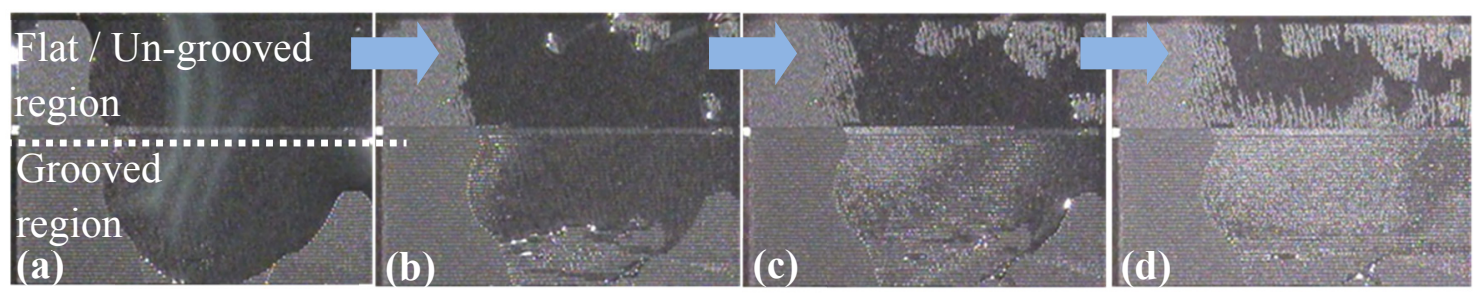

Figure 29. $(a-d)$ Comparison of drainage of an oil puddle from a grooved and un-grooved flat region on the sample blown by a Nitrogen air jet. As can been seen, most of the oil drains effectively along the grooves while a lot of it gets pinned on the nano-coating.

Immediately after the fabrication of the surface structures, the lubricating liquid is made to wet/passivate the entire surface. In doing this, the secondary liquid droplet to be manipulated on the surface is not in direct physical contact with the underlying structures on the surface and is immiscible with the liquid film that passivates the solid surface. This configuration causes the secondary liquid droplets to slip on the liquid-passivated surface. These surfaces are characterized by very low anisotropic sliding angles (across the ridges: $\sim 7^{\circ}$ and along the ridges: $\sim 5^{\circ}$ ) and contact angle hysteresis (across $\sim 5^{\circ}$ and along $\sim 3^{\circ}$ ). Further still, the novel mobility mechanism imbibed within these surfaces aids in the movement of secondary liquid droplets across these surfaces electrically. For this, the electrodes that aid in the actuation of liquid droplets across the surface have been incorporated such that they act as templates in the formation of the micro structures (microgrooves) during the fabrication process. The surface mobility feature facilitates the 
movement of liquid droplets on non-tilted surfaces, a technique that is not possible on natural non-wetting surfaces such as lotus plant leaves and the inner surface of the pitcher plants as well as similar artificially created omniphobic surfaces, which rely on gravity to make droplets roll off.

\subsection{Conditions needed to develop slippery surfaces}

One of the attributes desired of the lubricating liquid imbibing the underlying microgrooved surface is that it should wet the surface entirely and remain 'locked' into the substrate over extended periods of time. Also, the secondary liquid droplets should at no point displace the lubricating fluid and come in direct physical contact with the micro/nano features on the surface. In the event that this displacement occurs, the secondary liquid adheres very strongly and gets pinned onto the micro/nano textures on the surface which is not a desirable feature. While fabricating the surface, the micromachining step which employs reactive ion etching (RIE) increases the roughness parameter $r$ while etching into the surface by forming a fibrous network between the electrodes. Furthermore, the nano particulate fluoropolymer coating further increases the roughness during the final processing step. Increasing the roughness $r$ helps the lubricating liquid 'lock' into the surface more effectively as compared to a smooth micro-grooved surface. Details about the effectiveness of the nano particulate coatings have been presented in the following section.

It has been previously established [53] that the conditions required for a stable lubricating film which does not get displaced by the secondary liquid are determined by comparing the total interfacial energies of micro grooved surfaces that are completely wetted by the 
secondary liquid $\left(E_{A}\right)$, with the same micro grooved surfaces wetted by the lubricating liquid $\left(E_{2}\right)$ with or without the secondary liquid $\left(E_{1}\right)$. To ensure the solid is wetted preferentially by the lubricating fluid at all times, one should have $\Delta E_{1}=E_{A}-E_{1}>0$ and $\Delta E_{2}=E_{A}-E_{2}>0$ where $E_{A}$ is the non-desired energy state of the slippery surface. The non-desired state is a state where the slippery surface is primarily wetted by the secondary fluid to be repelled and not the lubricating fluid. The idea behind forming stable slippery surfaces is to keep the non-desired state in a higher energy state at all times.

\subsection{Mechanisms governing slippery surfaces}

Patterns on a hydrophilic solid at a scale much smaller than the capillary length (above which gravity dominates surface tension effects) can induce superhydrophilicity; the same is true for oleophilic surfaces. Once dropped on the surface, the micro/nanostructures guide the lubricating oil in a manner similar to wicking while the nano fibrous network further increases the wettability of the surface [68]. Furthermore, the nano particulate fluoropolymer coating increases the solid-liquid contact area dramatically and decreases the apparent contact angle of the lubricating film. 


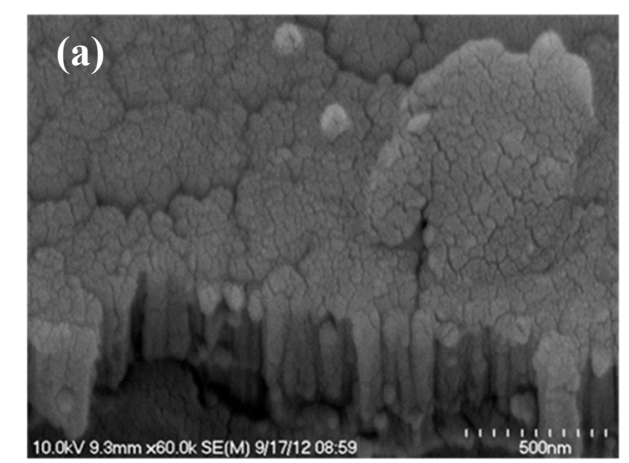

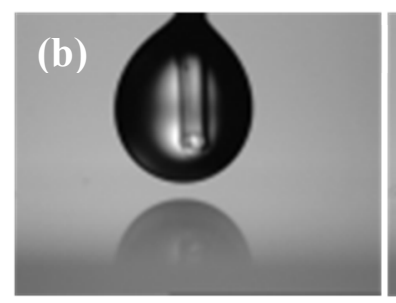

Before contact

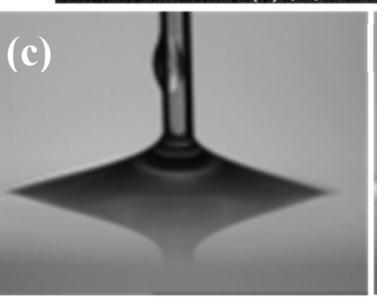

Immediately upon contact

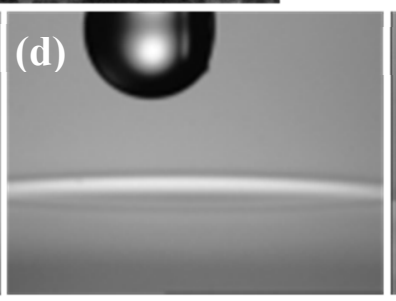

Few seconds

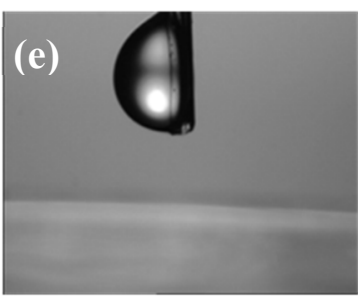

1minute later

Figure 30. (a) SEM images of the nano particulate/Cytop coating on the electrodes. Silica nanoparticles (5wt \%) with average diameter of $100 \mathrm{~nm}$ were uniformly dispersed in a 2 $w t \%$ Cytop solution before application onto the surface. Silica nanoparticles (100nm) were chosen as they are dielectric and do not cause electrical shorting between electrodes and ground. (b) FC-70 droplet before contact. (c) Spread of FC-70 droplet upon contact. (d) and (e) Continuous spreading of FC-70 forming a very thin liquid film upon contact. 


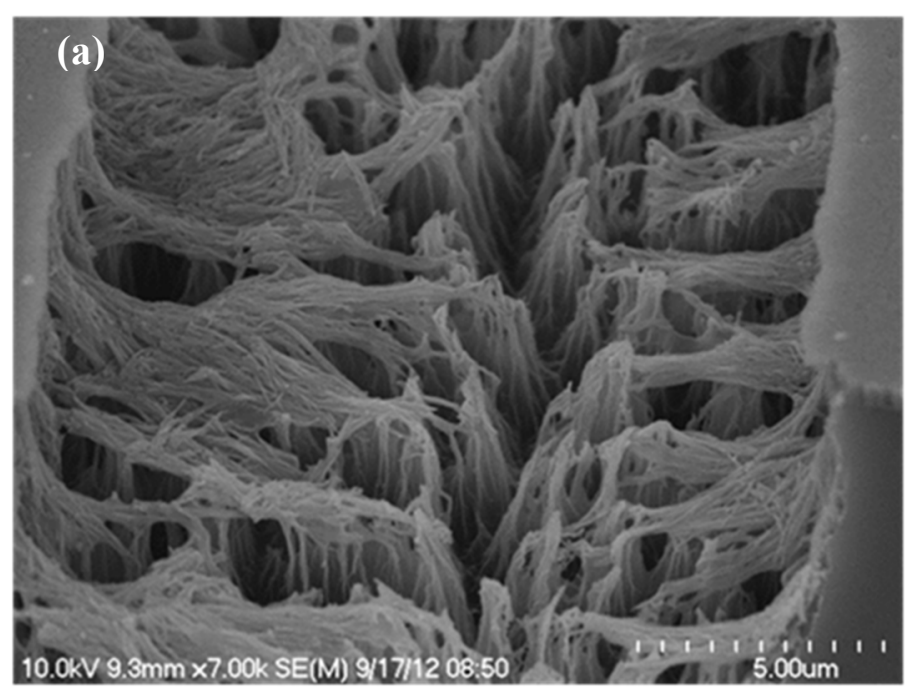

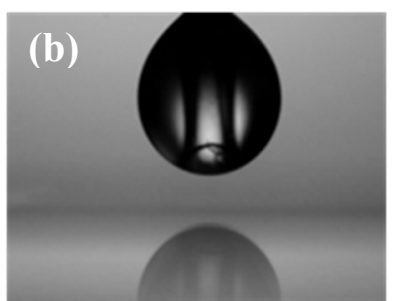

Before contact

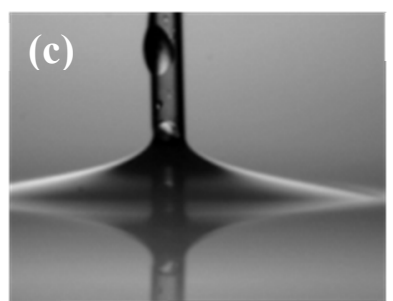

Immediately upon contact

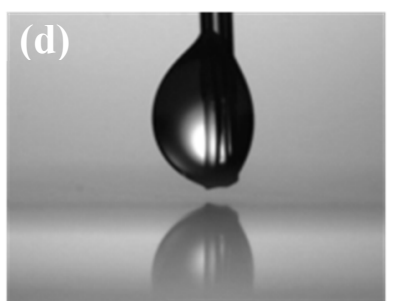

Few seconds

later

Figure 31. (a) SEM images of the plasma induced nano roughness within the grooves. The nano roughness is used to lock in the lubricating liquid. Roughness is further enhanced by the application of the Cytop/nano-particulate coating onto these nano structures. (b) FC70 droplet before contact. (c) Spread of FC-70 droplet upon contact. (d) Wicking of FC70 liquid into these structures immediately upon contact. The absence of a liquid film on the surface confirms the wicking of the liquid into the surface.

An increase in the effective contact area further enhances the capillarity of the surface. A sufficient increase in capillarity moves the contact line in the lateral direction so that a capillary liquid flow occurs [69]. This can be visually observed as soon as a drop of the lubricating oil is placed on the surface. Wicking causes the lubricating liquid to wet the entire surface instantaneously. (Figure 30 and Figure 31). Only after the entire surface is wetted by the lubricating liquid, the surface attains its 'slippery' properties which make it suitable for secondary liquid manipulation. The presence of the thin lubricating film between the secondary liquid droplet and the substrate allows the droplet to spread over 
the grooves while being in the Wenzel state but prevents the droplet from adhering and getting pinned to the micro/nano structures on the surface.
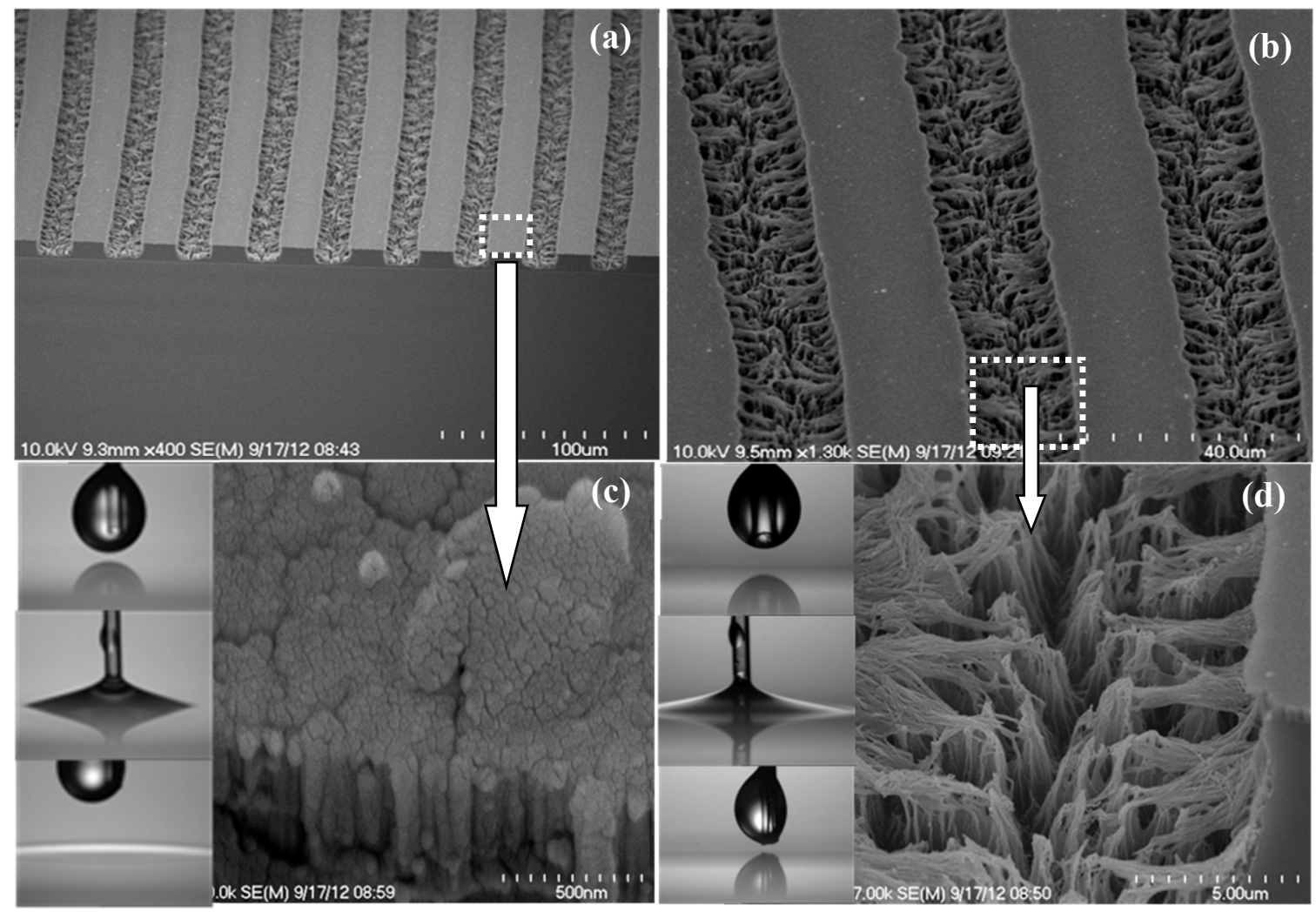

Figure 32. Scanning electron microscopy (SEM) characterization of slippery surfaces. (a) Cross section of the surface cleaved across the micro-grooves. (b) Zoom-in of (a). (c) SEM images of the nano-particulate coating used for promoting the wicking of the lubricating fluid on the electrodes. (d) SEM image of the plasma etched inter electrode spacing used for 'locking' the lubricating fluid into the surface.

In a number of studies dealing with morphologically structured hydrophobic surfaces, the Cassie-Baxter state appears to have a higher energy as compared to the Wenzel state. In other words, complete wetting of the microgroove surface structures corresponds to a situation of thermodynamic equilibrium. Nevertheless, in the absence of the lubricating liquid, the metastable Cassie-Baxter state is frequently observed on these surfaces when the droplets are deposited normal to the surface effectively experiencing a local energy minimum. This corresponds to the superoleophobic nature of the surface. With the 
lubricating liquid present (wetted state), air pockets might sometimes be trapped between the interface of the lubricating and the secondary liquid droplet. The presence of displaced air pockets from within the grooves and between the liquid-liquid interfaces has been observed optically during actuation. These air pockets prevent the suspended secondary liquid droplet from readily transitioning to the Wenzel state thereby preventing the wetted surface of the droplet from having a continuous liquid-liquid interface. Thus cushioning of the secondary liquid droplet arises from liquid pockets (lubricating liquid) and to a smaller extent from air pockets between the two liquid interfaces. However immediately during actuation, the secondary liquid comes in direct contact with the lubricating liquid thereby displacing air pockets and being cushioned by only the lubricating liquid.

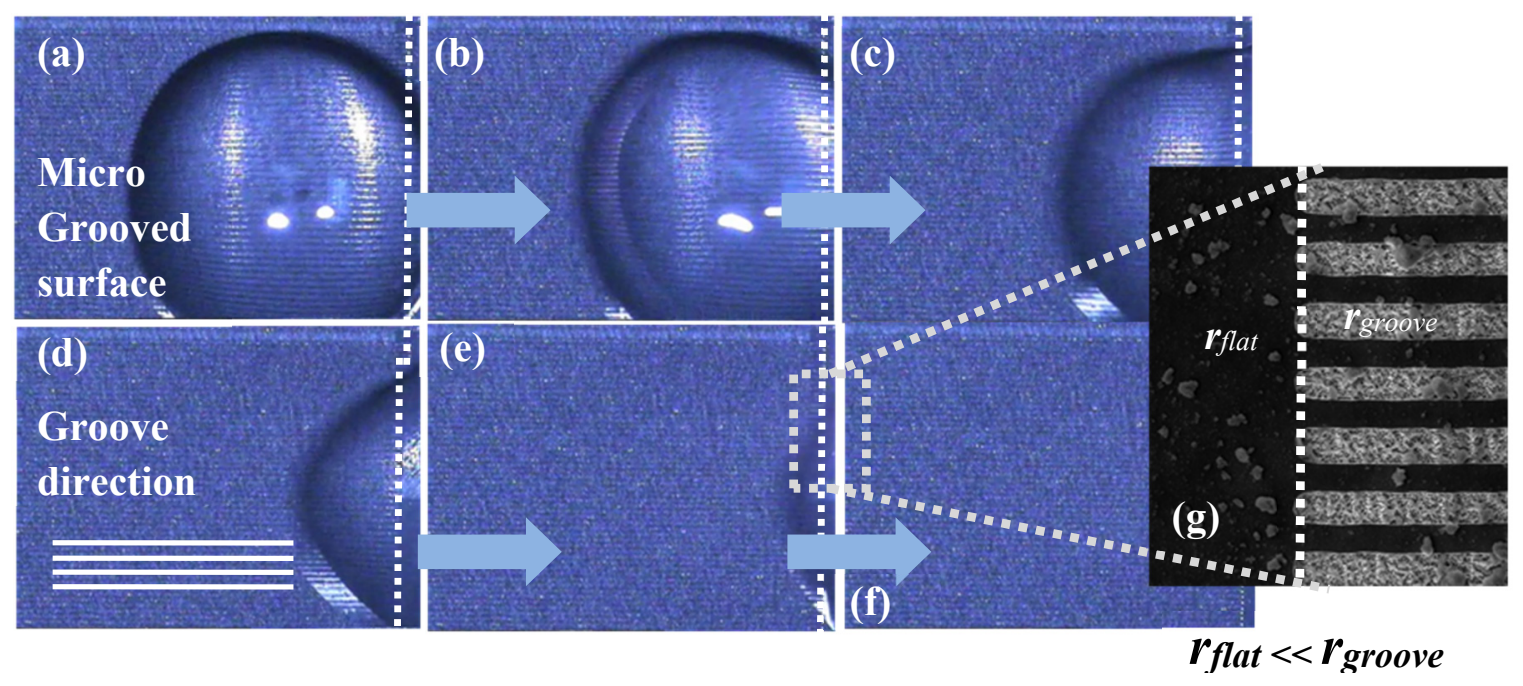

Figure 33. Differential Laplace pressure initiated droplet movement. (a-f) Liquid droplet (castor oil) seen transitioning from a position on the micro grooved region to a position on the flat region due to the Laplace pressure imbalance. (g) SEM image inset of the border between the flat region and the micro grooved region. On the flat region, roughness parameter rflat is greater than 1 but much smaller as compared the micro grooved region where $r>>1$.

The slippery effect of these surfaces is better visualized when a secondary liquid droplet is dropped onto the surface between the micro-grooved area and a flat (non-grooved) area. 
As soon as the droplet contacts the flat region (Figure 33), it is immediately pulled toward the micro-grooved region until its wetted surface resides completely on the flat region without any liquid remnants left behind on the micro-grooved surface. Such motion typically results from the non-equilibrium Laplace pressure generated on opposite sides of the droplet. Similar observations [70] were made by other researchers with aqueous droplets on flat and roughened polydimethylsiloxane (PDMS) surfaces. Aqueous droplets deposited across the boundary between the flat and rough/micro-structured regions on a surface moved away from the boundary towards the flat region. Such motion has been attributed to the differential pressures existing within the droplet when deposited at the boundary between flat and rough/micro-structured regions due to the different radii of curvatures arising from contact angle differences [70]. Differential pressures which are governed by the Laplace-Young equation cause the movement of microscale liquid droplets (Figure 33) such as oil droplets to move across the boundary between the microgrooved and flat regions. This is the first time that droplet motion arising due to differential Laplace pressures alone on non-tilted slippery surfaces has been demonstrated. Similar to previously fabricated slippery surfaces, these surfaces when tilted exhibit very low sliding angles and contact angle hysteresis but with slightly higher than previous reported values. This is because of the anisotropic morphology of these surfaces needed for the electrical actuation of secondary liquid droplets. Furthermore, the slippery effect on these surfaces can be extended to non-tilted surfaces using electrical actuation schemes which distinguishes them from various other demonstrated slippery surfaces. Dielectrophoretic forces are the primary mechanism by which liquid droplets are actuated across the surface. Dielectrophoresis (DEP) is an example of the ponderomotive effect, the basis of which is 
the force exerted on dipoles by a non-uniform electric field [71]. The dipoles of individual molecules in a liquid tend to collect in regions of high electric field intensity. While designing these surfaces, a non co-planar design - referred here as 'out-of-plane' electrode design - was implemented. This design causes the electric field lines to extend from the ridge tops to the underlying ground electrode. This non-uniform electric field within the micro-grooves, created by the out-of-plane electrodes, establishes a hydrodynamic equilibrium that the secondary liquid rushes to fill when voltage is applied. Once the equilibrium is reached, flow stops.

(a)

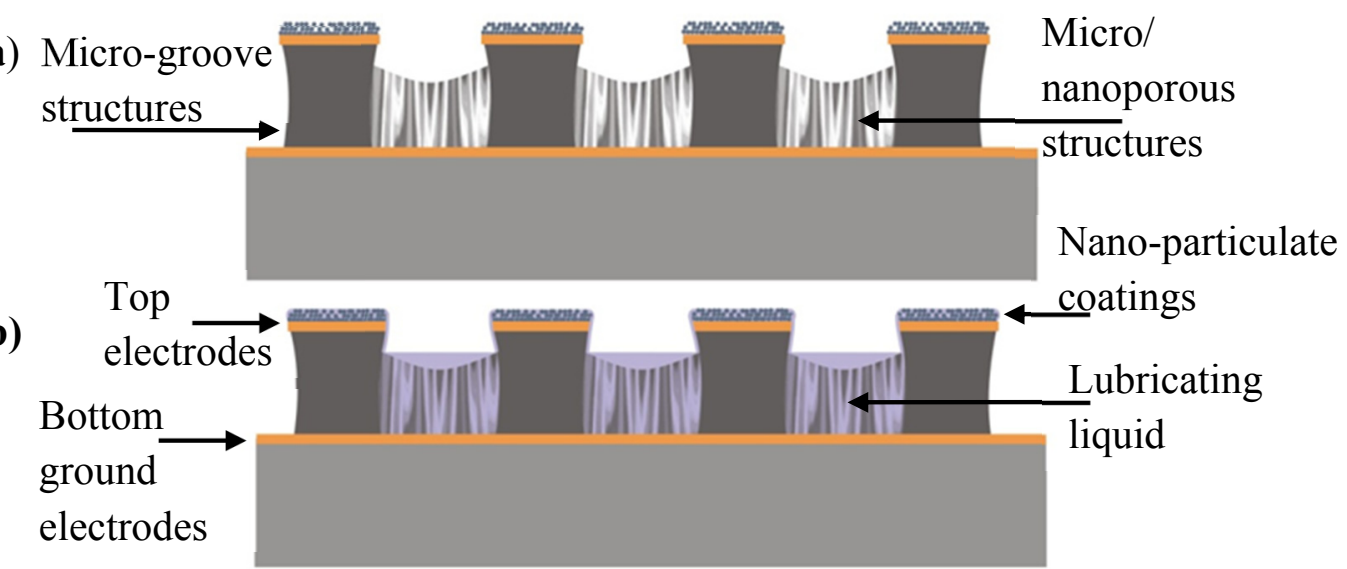

(c)

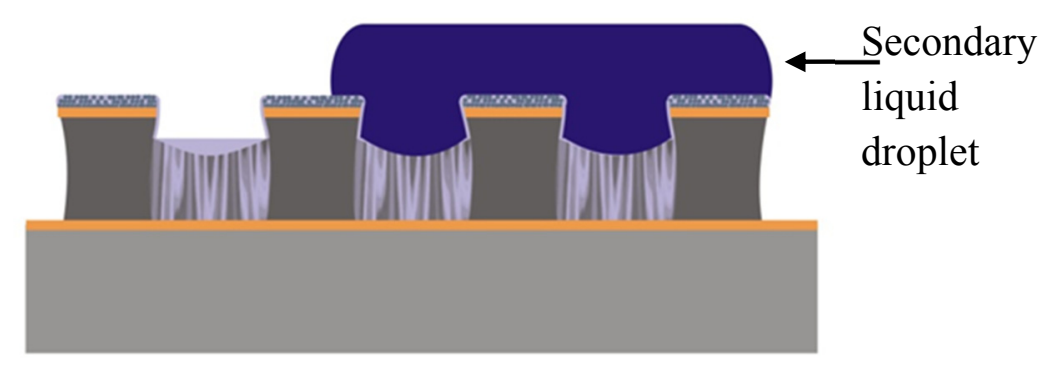

Figure 34. Cross-sectional schematic view of slippery surface. (a) Schematic of slippery surface embedded with electrodes for the driving of secondary liquid droplets on the surface. (b) Schematic of the slippery surface with the lubricating fluid locked into its surface. (c) Schematic of the slippery surface with a liquid droplet on its surface and the lubricating fluid locked into it. Liquid Dielectrophoresis (L-DEP) is used as the actuation mechanism in driving the liquid droplet across the surface. 
Non polar liquids such as silicone oil $(\gamma=20.4 \mathrm{mN} / \mathrm{m})$, castor oil $(\gamma=39 \mathrm{mN} / \mathrm{m})$ and hexadecane $(\gamma=27.7 \mathrm{mN} / \mathrm{m})$ were successfully actuated on these surfaces. A reversible change in contact angle has also been observed on these slippery surfaces up to a certain threshold voltage. The observed phenomenon is similar to reversible electrowetting of liquid droplets in a secondary liquid medium; the lubricating liquid in our case having the same function or playing the same role as the secondary liquid in a two-phase electrowetting case [72]. For the case of silicone oil (Figure 35a,b), a reversible change in contact angle has been observed up to an actuation voltage of $290 \mathrm{~V}$ within a few seconds after the voltage is turned off. A similar observation is made in the case of castor oil (Figure $35 \mathrm{c}, \mathrm{d})$. In the absence of any electric fields, the lubricating liquid within the micro grooves and on the ridge tops prevents the secondary liquid droplet from having direct contact with the underlying solid surface. With the application of an electric field, the dielectrophoretic forces generated between the electrodes draw the secondary liquid further into the grooves. This causes the secondary liquid droplet's contact line across the ridges to momentarily advance further giving an appearance of a change in contact angle. We believe this mechanism plays a role in the movement of liquid droplets across the surface. 
At 0 V

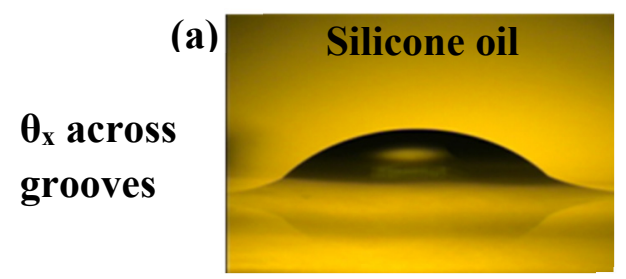

Reversible

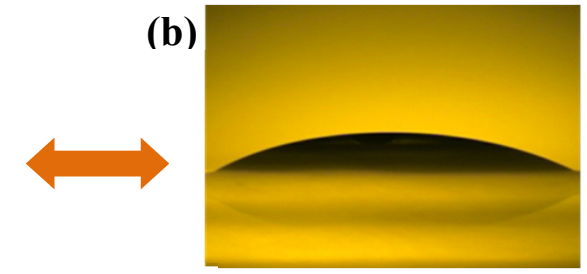

(d)

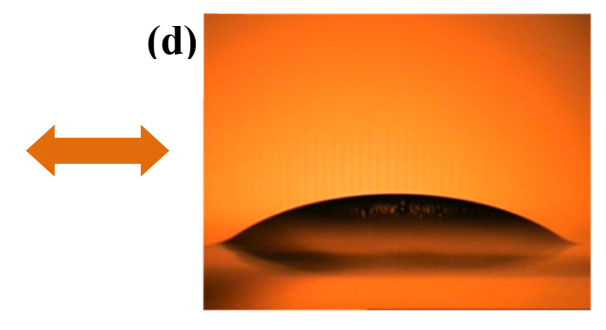

At 290 V

$\boldsymbol{\theta}_{\mathrm{x}}$ across grooves

\section{(c)}
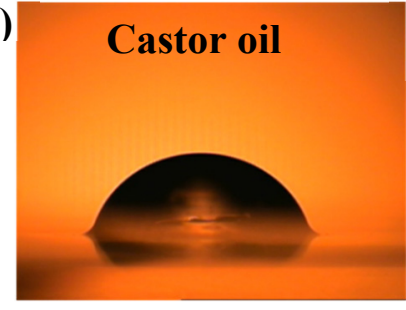

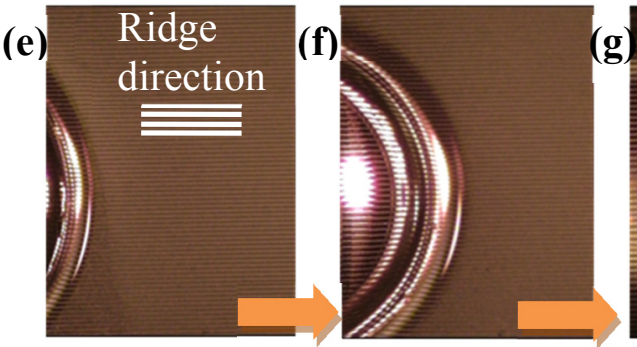

Driving Voltage ON
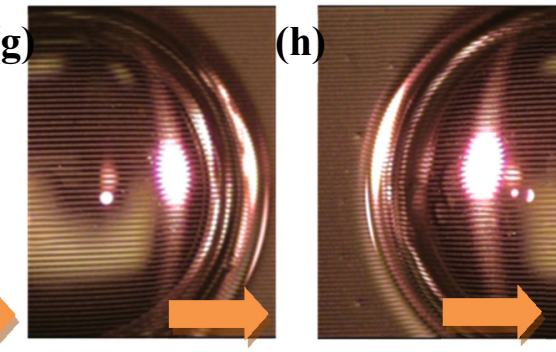

Driving Voltage OFF

Figure 35. $(a-d)$ Reversible change in contact angle and droplet actuation. Reversible change in contact angle observed on the slippery surfaces up to a threshold voltage of $290 \mathrm{~V}$ for the case of silicone oil. (e $-g)$ Electrically actuated oil droplet (castor oil) transitioning from one electrode to the next while the driving signal is ON. (h-i) Oil droplet (castor oil) continues to glide on the slippery surface with driving voltage OFF.

Aqueous liquids were also tested for electrical actuation across these surfaces. However no actuation was observed in the case of aqueous liquids with the exception of synthetic A+ blood. Even with an $\mathrm{AC}$ actuating voltage up to $400 \mathrm{~V}$ at $30 \mathrm{kHz}$ as demonstrated for the L-DEP actuation of water droplets on a flat surface [71], the actuation of pure de-ionized (DI) water droplets was not observed on these slippery surfaces. For the purpose of actuation of aqueous liquids such as blood, the surfaces were coated with a very thin layer of Parylene $(\sim 300 \mathrm{~nm})$ before the application of the nano-particulate fluoropolymer 
coating, to prevent electrolysis of the fluid's aqueous components. One interesting aspect observed while trying to actuate aqueous droplets such as blood or water across these surfaces was that these droplets did not exhibit any liquid loss through evaporation over several hours. In the case of droplets of aqueous liquids as well as oils placed on the slippery surface, a thin layer of the underlying lubricating liquid appears to coat the droplet. It's a known phenomenon that low surface tension liquids such as oils tend to spread and 'cloak' aqueous droplets of higher surface tension [73]. Cloaking is not always a desirable feature as it can cause the progressive loss of the lubricating fluid through entrainment of aqueous droplets as they move across the surface [73]. On the other hand, cloaking by low surface tension lubricating fluids can be desirable by preventing the evaporative loss from taking place on the secondary liquid droplet's exposed surface. The spreading coefficient

$S$ which is given by $S_{\text {oil-water }} \equiv \gamma_{\text {watet-air }}-\gamma_{\text {watet-oil }}-\gamma_{\text {oil-air }}>0$ indicates the presence of a cloaking film. Cloaking was observed in almost all the liquids tested on the surface which included non-aqueous as well as aqueous liquids. 


\section{Synthetic A+ blood}
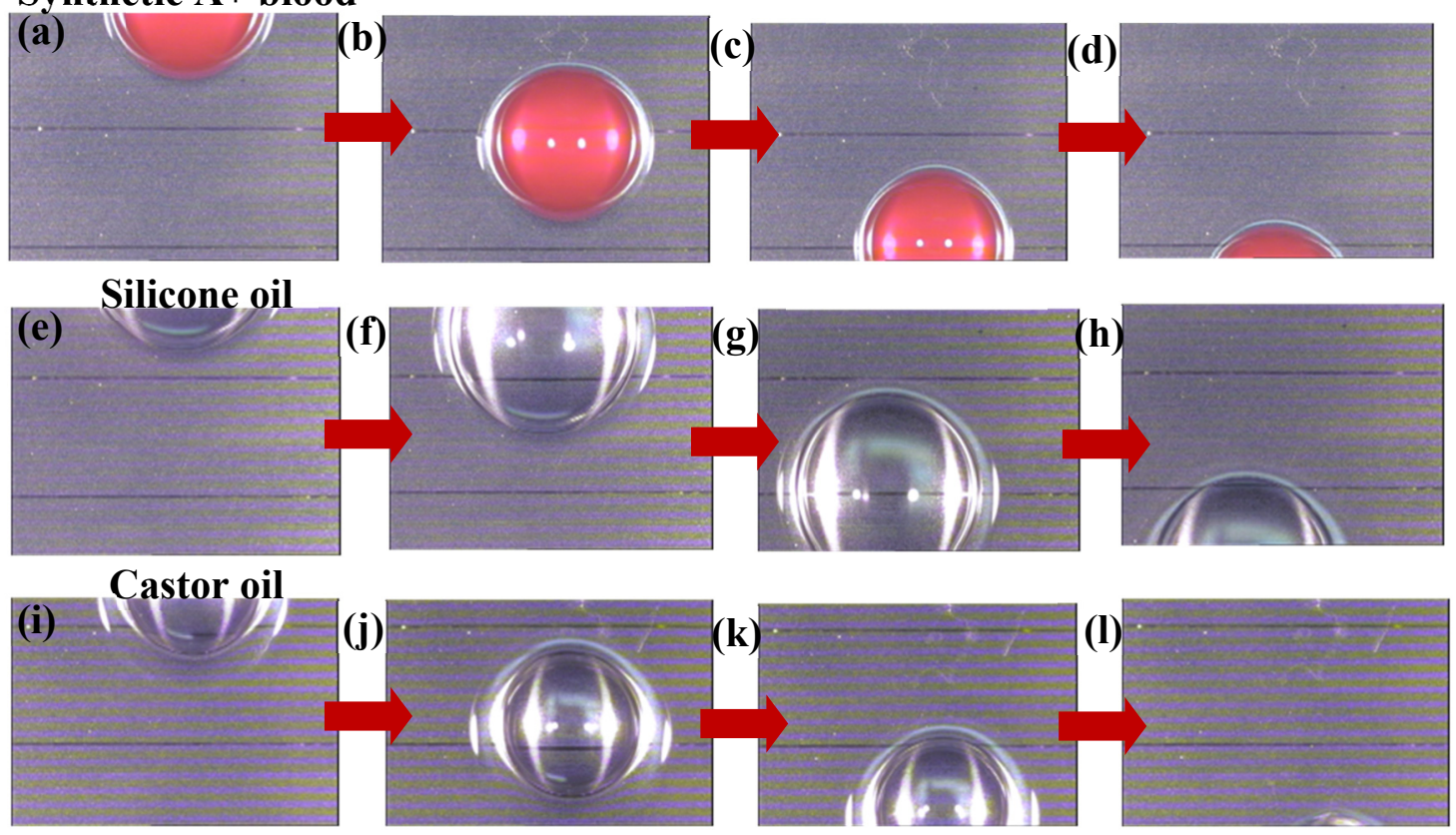

Figure 36. Actuation of liquid droplets on surface. (a-d) Actuation of synthetic A+ blood droplet across surface. (e-h) Actuation of silicone oil droplet (50 Cst) across surface. (i-l) Actuation of castor oil droplet across surface.
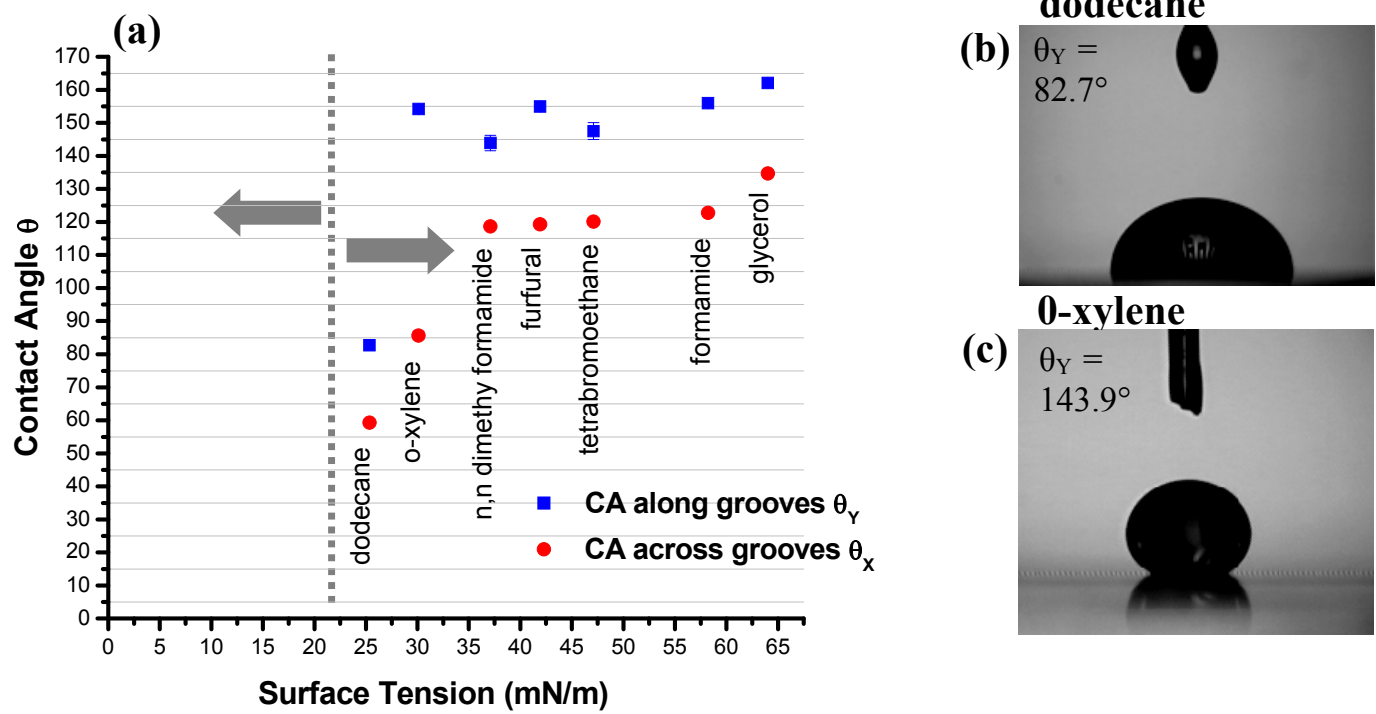

(c)

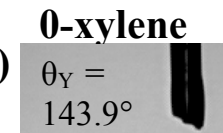

Figure 37. Slippery surface characterization using contact angle measurements. (a) Contact angles of different test liquids on the slippery surfaces without the lubricating liquid - $\theta_{X}$ across the grooves and $\theta_{Y}$ along the grooves. All the test liquids with the exception of dodecane, exhibit a very high contact angle while measured along the grooves which suggests that they reside in the Cassie state with trapped pockets of air within the micro grooves. (b) Contact angle $\theta_{Y}$ of dodecane. (c) Contact angle $\theta_{Y}$ of o-xylene. 
In subsequent tests, the surface was characterized using different test liquids covering a wide range of surface tensions. Contact angles of secondary test liquids with surface tensions ranging from $18 \mathrm{mN} / \mathrm{m}$ to $80 \mathrm{mN} / \mathrm{m}$ were measured on these surfaces in the absence of the lubricating liquid. Since these surfaces are anisotropic in their wetting characteristics because of the presence of parallel micro grooves along the surface, the surfaces were characterized in two different directions - with the line of sight parallel and perpendicular to the grooves. It was observed that the surface exhibited anisotropic superoleophobic characteristics with low surface tension liquids similar to directional superoleophobic surfaces demonstrated previously [74]. With the exception of dodecane $(\gamma=25.35 \mathrm{mN} / \mathrm{m})$, all the other secondary test liquids were seen to reside in the Cassie state with trapped pockets of air underneath as evidenced by the very high contact angles. In the case of dodecane, as soon as the droplet contacts the surface, it momentarily exhibits a very high contact angle (characteristic of the Cassie state) which decreases rapidly below $\theta_{\mathrm{Y}}=90^{\circ}$. This occurs due to liquid loss in the sessile droplet because of liquid propagation along the ridges and into the grooves of the surface. As the liquid propagates along the ridges, it transitions to the Wenzel state which has a considerably lower contact angle than when in the Cassie state.

\subsection{Characterization of optical properties of slippery surfaces}

The optical properties of these surfaces were studied using UV-VIS spectroscopy, as in certain applications optical transparency may be a desirable feature. For such types of applications where optical transparency is needed, the metal electrodes that are initially used as micromachining templates can be replaced by transparent electrodes such as Indium Tin Oxide (ITO). Patterned ITO electrodes could also have been used for 
subsequent microgroove formation using selective Reactive Ion etching (RIE) of the SU8 layer. For optical testing, the aluminum electrodes were etched away immediately after the microgroove formation and the sample was optically characterized using UV-VIS spectroscopy under different conditions. This was done to mimic the similar optical characteristics that the slippery surface would possess if ITO electrodes were used. It was observed that the slippery surface had similar optical characteristics with and without the application of the nano-particulate fluoropolymer coatings with transmittance around $60 \%$ before the application of the lubricant. As soon as the surface was lubricated/passivated with the lubricating oil (FC-70), the transmittance increased to about $80 \%$. The roughness of the coatings within the microgrooves and on the top electrodes is small enough to prevent scattering of visible light. Furthermore, the difference in refractive indices is reduced due to the infused lubricating liquid which transforms the solid-air interface to a solid-liquid interface. The filling of sub-micron/nano sized features - especially those that are larger than $100 \mathrm{~nm}$ and cause significant light scattering - by the lubricating liquid, effectively enhances the optical transparency. This data highlights the possibility of using slippery surfaces in opto-microfluidic applications where transparency and electrical manipulation of fluids is necessary. 
(a)

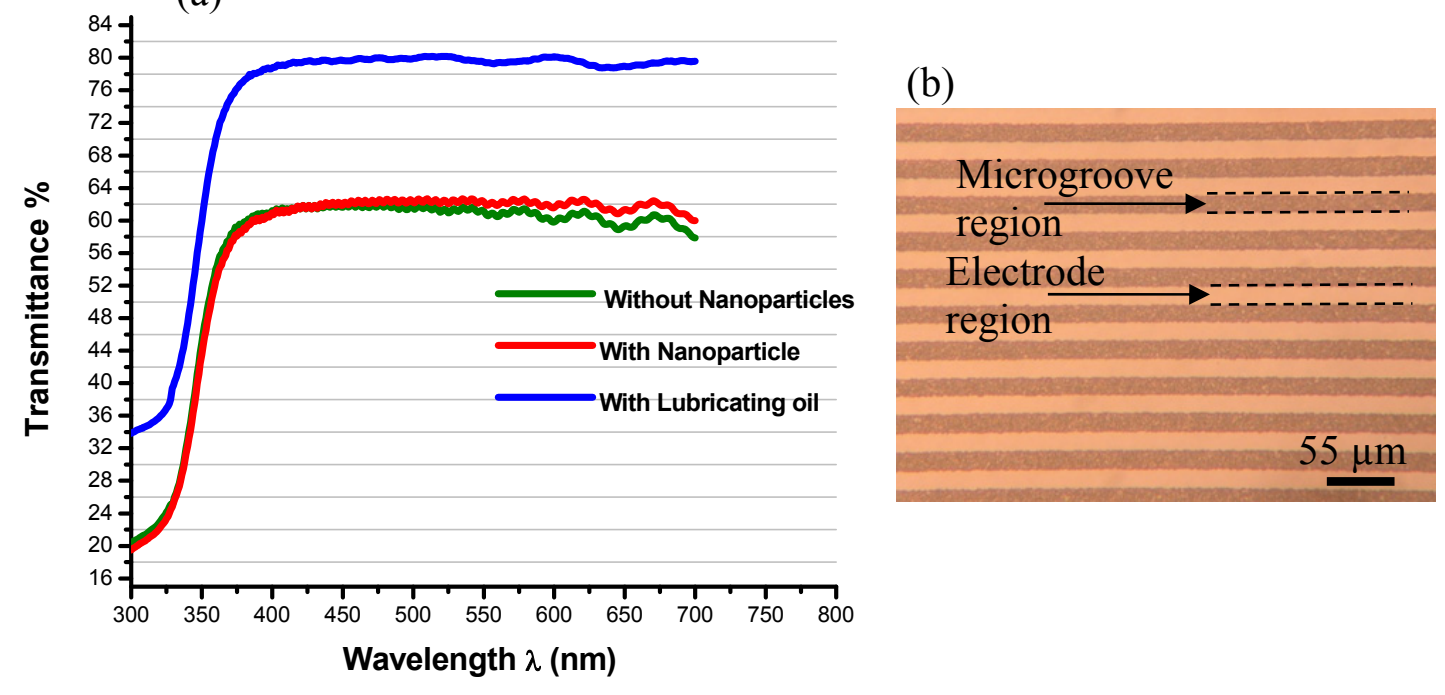

(c)

(d)

(e)

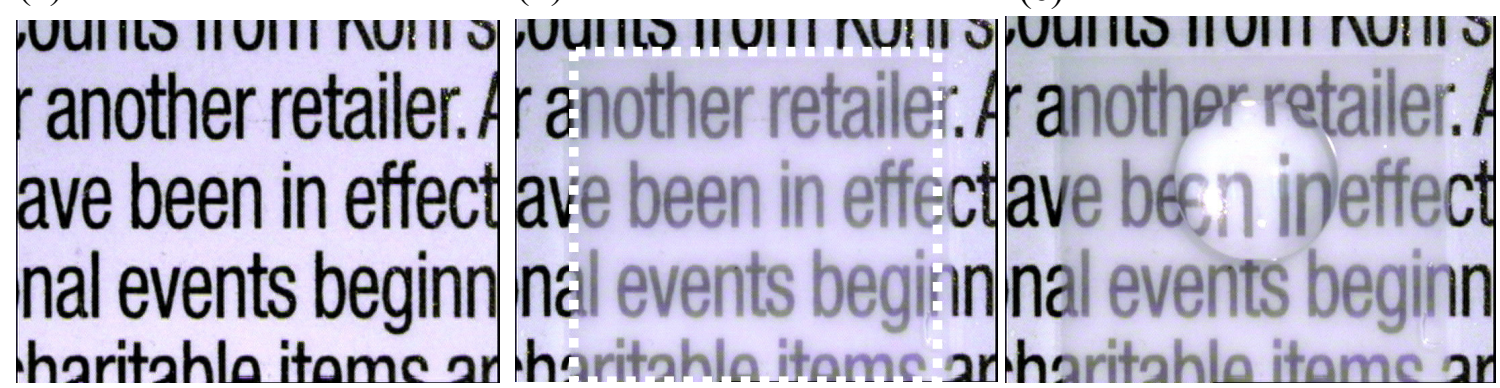

Figure 38. Optical characteristics of slippery surfaces. (a) Optical transmission characteristics of slippery surfaces for the different cases of micro grooved surface without the nano-particulate coatings and lubricating fluid, with the nano-particulate coating but without the lubricating fluid, and with the lubricating oil/nano-particulate coating. (b) Optical image of slippery surface with the nano-particulate coating but without the lubricating fluid. (c) Image of plain text on paper. (d) Image of plain text on paper under the slippery surfaces. (e) Image of plain text on paper under the slippery surface with an oil droplet placed on the slippery surface.

Inspired by various parts of the pitcher of the Nepenthes pitcher plant, the driving of dielectric liquids on an artificially created slippery surface was demonstrated. Liquid dielectrophoresis (L-DEP) was utilized for enhancing droplet motion across the surface with the help of the slippery properties exhibited by these surfaces. These surfaces, when damaged, can self-heal and continue to remain slippery and allow electrical actuation 
across the surface. Additionally, these surfaces which are omniphobic to both aqueous as well as non-aqueous/oily liquids exhibit excellent optical characteristics while being almost transparent in the UV range. We believe these surfaces have the potential for labon-chip applications (LOC) where superhydrophobic and superoleophobic surfaces could not be previously used.

\subsection{Fabrication details and methods for slippery surfaces}

Fabrication starts with the patterning of a metal layer deposited on a bare pre-cleaned glass substrate followed by the deposition of $10 \mu \mathrm{m}$ thick SU8 sacrificial layer that is crosslinked. A second metal layer is then deposited on this SU8 layer which is subsequently patterned forming the top electrodes and these act as a template in the formation of the microgrooves. The microgrooves are formed by etching using a Reactive Ion Etch (RIE) in an Oxygen environment at $150 \mathrm{mT}$ and $200 \mathrm{~W}$ RF power. A nanoparticle colloid formed using $100 \mathrm{~nm}$ size silica nanoparticles dispersed in a Cytop solution is then spin coated (3x) onto the reactively ion etched (RIE) surface and cured. The lubricating liquid which is FC70 is then dropped onto the micro-grooved surface and any excess liquid is blow dried away. This completes the liquid infused surface which is very slippery to other liquids such as silicone oil and castor oil. A low surface tension liquid, 3M Fluorinert (FC-70, $\gamma=17.1$ $\mathrm{mN} / \mathrm{m}$ ) was used as the lubricating liquid on these surfaces. 


\section{Chapter 5}

\section{LONG TERM RELIABLE BIO-FLUID PRINTING WITHOUT NOZZLE FAILURES}

\subsection{Introduction}

Inkjet printing has been an important means in many evolutionary and progressive tissue engineering applications while developing various in-vitro models inexpensively and with high throughput. Despite the many advantages of inkjet printing of bio-fluids for tissue engineering applications, reliable and quality ejection of bio-fluids over extended periods of time has not been guaranteed due to various nozzle failures. Bio-fluids generally evaporate quickly at the tiny nozzles and cause clogging problems. In this article, we present a reliable contactless dispensing technology for bio-fluids without such clogging related problems. Stable and easy motion of a thin liquid film aided by nanostructures was adapted for a nozzle capping mechanism to prolong clean nozzle conditions over long periods of time. Reliable contactless delivery by printing various bio-fluids such as hydrogels and other protein solutions has been demonstrated without any nozzle failures.

\subsection{Inkjet printing for contactless bio-fluid delivery applications}

Synthesis of transplantable tissues has been enabled by combining the principles of engineering, physical sciences and medicine. Many tissues and organs can be especially synthesized in a laboratory setting before being transplanted into the human body $[4,75$,

76]. There have been huge breakthroughs in controlled production of tissue engineered skin, cartilage, bone, blood vessels, etc. [77-80]. 
Inkjet printing has many advantages for tissue engineering and proteomics applications. Recent advances in tissue printing offer the capability to quickly deliver cells and other biomaterials for building tissue structures with the aid of gels [81]. Especially, hydrogels have widely been used in many tissue engineering applications because hydrogels can provide an aqueous and porous environment for cells, and help cells retain their shape after printing. Hydrogels are protein and carbohydrate-based polymers and can extend cell viability by preventing drying and other harmful effects during and after fabrication processes [82]. In addition, inkjet printing can accurately align cells spatially. The importance of spatial alignment of cells has already been demonstrated in vitro while attempting to accurately mimic the in vivo microenvironment [83]. Conventional cellseeding methods are inadequate in the development of in vitro tissue test systems because the random placement of cells lacks the precision in spatial control, which enables better predictions of cell function and an overall increase in the stability of cell phenotype [84]. Also, deposited concentrations of hormones using inkjet printing can easily be modulated by accurately overprinting individual locations with dilute bio-fluids [85]. Moreover, the contactless delivery nature of inkjet printing aids in reducing contamination when dealing with purified materials. Many bio-fluids can be contactlessly deposited even onto non-flat surfaces. Furthermore, inkjet printing, particularly drop-on-demand techniques utilize pico to nano liter-sized quantities of buffered protein solutions which may be precisely dispensed to a designated sample space, with minimal waste. These low volumes allow researchers to sparingly use valuable proteins, such as purified enzymes, and place them in the desired location. Thus, inkjet printing has been employed in proteomics as a highthroughput tool for the preparation of samples for analysis (e.g. printing protein arrays) as 
well as for peptide synthesis [86]. One of the applications of inkjet assisted proteomics is in peptide mass fingerprint mapping and has become one of the most predominant methods for the identification of proteins $[87,88]$.

Although inkjet printing is very suitable for many biomedical applications described above, there are still many features limiting the usage of the inkjet printing technology. For example, inkjet printing has known problems of nozzle failures due to the dried residue build up by evaporative liquid loss at the nozzle. Thus, it is very challenging to use inkjet printing for long-term dispensing of biological samples due to the rapid evaporation speed of volatile bio fluids at the liquid-air interface in the nozzle region. In certain specific cases $[89,90]$, liquid handling of the matrix solution has been a limiting factor while using microdispensers for protein analysis using Mass Spectrometry. For example, hydrophobic matrices such as $\alpha$-Cyano-4-hydroxycinnamic acid ( $\alpha$-CHCA) dissolved in 50\% Acetonitrile (ACN) were seen to frequently crystallize in the dispenser nozzle [90]. In other specific applications, the buildup of salt scaling at the nozzle orifice leads to spatter within a printed pattern caused by erratic spraying of the bio-fluid in the form of satellite droplets during the various levels of clogging [91]. Nozzle clogging is a universal problem that most inkjet based bio printers contend with. Previously, various technical limitations including nozzle clogging and sustainability issues of the printed tissues were investigated by researchers [92]. Many logistical barriers faced by inkjet printing technology for complex and 3D tissues were also addressed [93]. Nozzle failures appear to be exacerbated when a bio-fluid causes clogging as well as leaves residues within the nozzle chamber [93]. It can also easily cause significant volume loss, concentration, osmolarity and even viscosity 
change of the bio-fluids. In this study, we demonstrate that very reliable bio-fluid printing for extended periods of time without any aforementioned nozzle failure modes.

A review of the current mechanisms that are used for preventing clogging in inkjet printhead nozzles is presented in the following sections before introducing a 'Sliding liquid drop' (SLID) shutter technology for capping the nozzle. This SLID mechanism has been shown to prevent clogging and crystallization of bio-fluids at the nozzle. Furthermore this technology can prevent the oxidation of miniscule amounts of expensive bio-fluids such as purified proteins while in the printhead.

\subsection{Sliding Liquid Drop (SLID) shutter technology for use in bio-printers 5.3.1 Currently adopted techniques for clog-free ejection}

Several techniques for reducing clogging have been reported in literature. The use of electromechanical valves $[94,95]$ using pressure sources for dispensing hydrogels and other bio fluids, in bio-printers has been reported. This technique allows the adjustment of dispensing volumes by controlling the duration of the microvalve operations especially in the case of more viscous fluids. These valves prevent changes to viscosity, phase and osmolarity of the bio-fluids from within the reservoirs. However, these valves, while preventing volume loss of the bio-fluids in the reservoir, can potentially be clogged by evaporation of remnant bio fluids in the nozzle region especially if crystallization occurs. In another approach, the prevention of evaporation induced clogging by increasing the viscosity of the solution with the help of viscosity modifiers such as glycerin in concentrations as high as $40 \%$ [96] has also been reported. This helps by mitigating the evaporation of more volatile bio-fluids. However, induced viscosity modification with the 
use of reagents, can sometimes prove to be detrimental because of associated $\mathrm{pH}$ changes especially when printing bio-fluids such as Collagen. For example, it was observed that when printing collagen solutions at a $\mathrm{pH}$ of 5.5 , the viscosity increased dramatically due to collagen fibril aggregation [97]. Furthermore, it was observed that the collagen solutions at this $\mathrm{pH}$, became cloudy due to the onset of partial gelation. These observations correlated with the researchers' previous printing experience wherein they observed a high incidence of nozzle clogging when printing solutions of this $\mathrm{pH}$. In another example, when a serumfree cell culture medium was used as a bio-fluid, nozzle failure resulted in a relatively short time [91]. The failure was attributed to clogging by adsorbed proteins and cellular components, in addition to aggregated cells. However, nozzle failure was observed by the same researchers even while printing simple salt solutions. The build-up of salt scale as fluid evaporates at the nozzle and which results in degraded patterning, erratic cell ejection or complete nozzle failure, was identified as the failure mechanism. In their study [91], ethylene diamine tetra-acetic acid (EDTA) was used as a bio-fluid additive to prevent salt scaling and cell aggregation during the bio-printing process [91]. Non-friendly reagents such as 'Instrument lubricant' were also implemented by them, for cleaning salt scale evaporation residues, at later times. One of their observations worth noting is that, the devices (inkjet nozzles) remained bacteriostatic for approximately a month after the use of lubricants for cleaning purposes. This observation of theirs highlights the fact that additives and cleaning solutions are not entirely bio-compatible. Yet another emerging trend for eradicating clogging in droplet ejectors $[98,99]$ has been the complete elimination of nozzles by using a nozzle-free acoustic ejector. Although the clogging of nozzles is no longer an issue for nozzle-less and reagent modified bio-fluids, evaporative effects can still 
lead to the modification in viscosity, molarity etc. Furthermore, the use of reagents is not always preferred and can be toxic to cellular function.

\subsubsection{SLID clog-free technology}

One simple technique for preventing clogging related issues is to simply close the nozzle when not in use. The use of an immiscible ad non-volatile liquid film such as the sliding liquid drop (SLID) to prevent bio-fluid evaporation, as in the case of matrix solutions as well as hydrogel solutions such as Collagen, can be a more effective technique than reagent usage. Moreover, a SLID at the nozzle which prevents evaporation induced bio-fluid loss can remain immune to the effects of stiction. The SLID is composed of a liquid material whose chemical properties are similar to elastomers such as Polydimethylsiloxane (PDMS) but which is much more pliable being in the liquid state. The SLID acts like a valve mechanism thereby preventing bio-fluid evaporation taking place at the nozzle. It can be electronically activated each time printing needs to be carried out. Moreover, it does not require any bulky setups for its operation, and does not entail complex fabrication schemes thereby increasing device portability and decreasing fabrication costs. Furthermore, nanostructures have been utilized for imparting a normally-closed nature to the SLID. We believe that this technology has immense potential for the long term reliable printing of biological fluids.

Previously, we introduced a microfluidic valve for evaporation control of volatile aqueous and ink liquids [87]. In that implementation, a top cover-slip with a central orifice positioned exactly above a microfluidic port was used. An immiscible, thin liquid film sandwiched between the two surfaces - the bottom ejector surface and the top cover-slip surface - was used as a microfluidic valve to the microfluidic port. This sandwiched 
microfluidic valve served the same purpose as the SLID - to cover the nozzle region when not in use. Furthermore, the liquid film sandwiched between the bottom microfluidic port and the top cover-slip central orifice, denoted the closed state of the valve; while the liquid film sandwiched away from the microfluidic port and top cover-slip orifice denoted the open state of the valve. Liquid droplets eject out of the microfluidic port and exit the top cover-slip orifice, while the microfluidic valve is open during operation. At all other times, the valve remains closed. In that prototype, the sandwiched liquid film which constituted the microfluidic valve, was spatially controlled electrically similar to the SLID. Although the microfluidic valve exhibited excellent performance in terms of evaporation reduction, valve response times were less than satisfactory. In the best case, valve opening and closing cycles were accomplished in 3-4 seconds each. For volatile liquids that have low vapor pressures, this technique is feasible. However for volatile liquids with high vapor pressures such as certain bio-fluids, the technique poses a problem. To have a quantitative understanding of the detrimental effects of fast evaporation rates, one should consider the fact that ten nanoliters of water evaporates within thirty seconds under a standard laboratory environment whereas ten picoliters of water evaporates in less than a second. These time frames are drastically reduced when working with highly volatile liquids such as Acetonitrile (ACN), which is a commonly used solvent in bio printing applications such as peptide synthesis. By integrating the SLID to the nozzle, the response times - valve open and valve close times, are almost a second each and are dependent upon actuation voltages, making it suitable for the ejection of highly volatile bio-fluids. This makes the SLID ideal for implementation in bio-fluid droplet ejectors and printheads for bio-printers. 
Another advantage in using the SLID is its strong nozzle capping mechanism. Nanostructures micromachined around the nozzle have a very strong affinity to the SLID material and keep the SLID anchored to the nanostructured region at all times. Furthermore, the anchoring mechanism induced by the nanostructures, secures a fixed spatial position to the SLID above the nozzle, immediately after power to the electrodes is cut off. This characteristic was absent in the previous prototype wherein the sandwiched microfluidic valve was vulnerable to mechanical shocks and could have different spatial positions in either state. Interestingly, the normally-closed nature of the SLID makes it ideal for the ejection of bio-fluids. Without this feature, electrodes would need to be fabricated around the nozzle to position the SLID above the nozzle, thereby closing it. This can be a serious problem as evidenced in some of our tests as most biological fluids are conductive in nature. Remnants of bio-fluid around the nozzle region, immediately after the ejection process can provide conductive paths between electrodes and cause shorting especially in the case of co-planar electrodes used in the current design. A material with a high dielectric breakdown could be used as a passivation layer over the co-planar electrodes but would come at a price of higher driving voltages. Apart from being better suited for the printing of bio fluids as compared to the older design, the fabrication of the current design is simpler. It eliminates the top cover-slip with the central orifice projected over the nozzle and which is separated from the ejector face plate with a $25 \mu \mathrm{m}$ spacer. Furthermore, this design eliminates all the problems associated with the top cover-slip such as alignment of the cover slip orifice hole with the ejector nozzle, and it's drilling. Moreover, this technique is less expensive in terms of cost of materials. A schematic of the open channel SLID implementation is shown in Figure 39. 

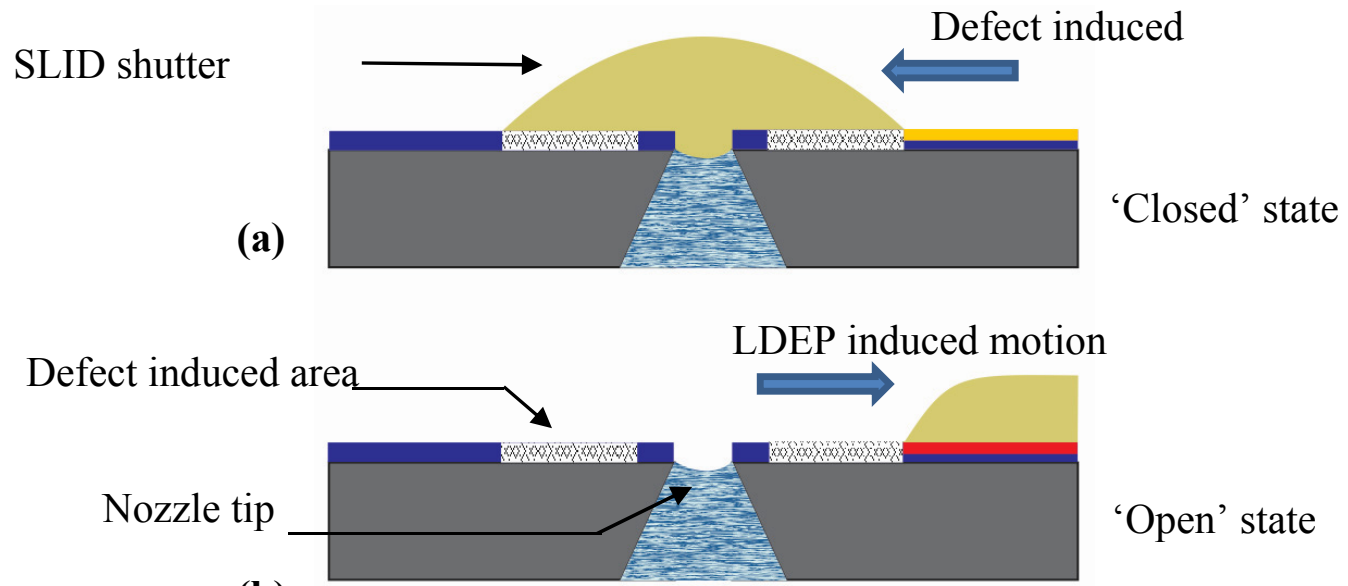

(b)

Electrode in $\left[\begin{array}{l}\text { ON state } \\ \text { OFF state }\end{array}\right.$

Figure 39. Schematic of the 'Sliding liquid drop' (SLID) integrated over the ejector nozzle. Actuation of the co-planar electrodes opens the SLID making it ready for ejection. De actuation of electrodes automatically closes the SLID because of the material's surfacetension shape restoring property.

\subsection{Fabrication details of ejector integrated with the SLID shutter}

The fabricated device consists of 4 main components integrated into a single system: 1) The LDEP system which consists of the co-planar electrodes on the micromachined ejector surface 2) The defect-induced / modified area on which the SLID shutter resides entirely at all times, except during the actuation of the co-planar electrodes 3) The fluidic chamber - formed by SU8 on a PZT ceramic transducer, which is below the ejector surface and 4) the Piezoelectric transducer with patterned electrodes on either side. 


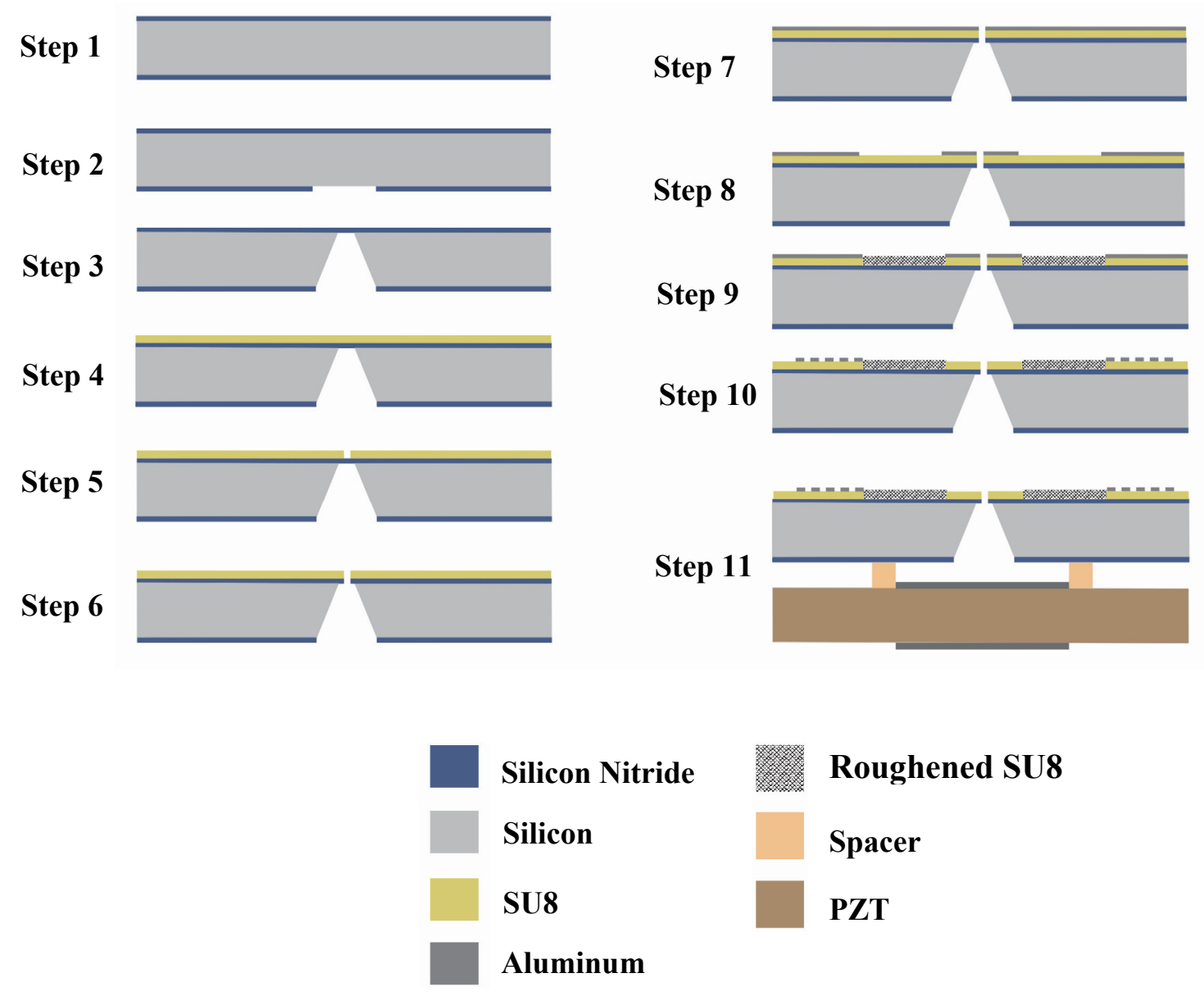

Figure 40. Image depicting the various steps of the fabrication process. Step 1: Silicon nitride wafer. Step 2: RIE etch of $S i_{x} N_{y}$. Step 3: KOH etch of $S_{i} N_{y}$. Step 4: Deposition of SU8 layer. Step 5: Patterning of SU8 layer. Step 6: RIE etch of exposed Six $N_{y}$ diaphragm. Step 7: Metallization. Step 8: Patterning. Step 9: RIE etch of exposed SU8. Step 10: Patterning. Step 11: Bonding to fluidic chamber on PZT.

The different fabrication steps in developing the normally-closed SLID shutter over the ejector nozzle are illustrated in Figure 40. Fabrication of the 'Sliding Liquid Drop' (SLID) shutter starts off by anisotropic etching of the unpolished side of Silicon using $\mathrm{KOH}$ through the $\mathrm{Si}_{\mathrm{x}} \mathrm{N}_{\mathrm{y}}$ openings (Step 2,3). This results in a 60-75 $\mu \mathrm{m}$ windows opening on the other side (polished side) of the wafer. After this, the $\mathrm{Si}_{\mathrm{x}} \mathrm{N}_{\mathrm{y}}$ diaphragms are left intact so that a conformal layer of SU8 can be patterned on the surface. For this a $10 \mu \mathrm{m}$ thick SU8 layer is spin coated, soft baked and then the nozzle region above the $\mathrm{Six}_{\mathrm{x}} \mathrm{N}_{\mathrm{y}}$ diaphragm is 
patterned into the SU8 (Step 4, 5). After the post exposure bake (PEB), the SU8 layer is developed and the nozzle over the diaphragms are realized. Immediately after this is done, the diaphragms are etched away using a CF4 plasma in a Reactive Ion etcher (RIE) system (Step 6). This is followed by a $1 \mu \mathrm{m}$ thick Al metal deposition using RF magnetron sputtering (Step 7). The Al layer is then patterned to expose a central region of the underlying SU8 layer around the nozzle (Step 8). This exposed region is then transformed to the defect-induced region, whose function will be explained below. A mild Oxygen plasma etch using the RIE system is used to create surface defects on the exposed SU8 region while all the other areas on the substrate remain defect free because of the $\mathrm{Al}$ masking layer (Step 9). The Oxygen plasma transforms the flat exposed SU8 surface to a roughened surface. After this step, the Al layer is further patterned to define the co-planar electrodes, whose function will be described below (Step 10). Stripping any residual photoresist after patterning of the co-planar electrodes is carried out by immersing the substrates overnight in a bath of $\mathrm{AZ} \mathrm{Kwik} \mathrm{strip} \mathrm{remover} \mathrm{at} 95^{\circ} \mathrm{C}$. After this, the substrates are washed in DI water, dried and then coated with Cytop. It is then cured at $180^{\circ} \mathrm{C}$ for an hour. The patterned PZT ceramic is bonded to the bottom side of the ejector plate using a general purpose epoxy with a $200 \mu \mathrm{m}$ thick SU-8 spacer in between (Step 11). After the ejector face plate is mounted on the fluidic chamber and bonded, the microfluidic valve material is loaded onto it. For this, a drop of silicone oil - used in the top covered devices, is dropped onto the circular region that is induced with surface defects. This region is called the modified / defect-induced region and has a great affinity to the SLID shutter liquid material. As soon as silicone oil comes in contact with the defect-induced region, it spreads and completely wets this region. For the proper functioning of the SLID shutter, the right 
amount of oil needs to be dispensed on the modified / defect-induced region. Any excess amount will cause the SLID shutter to malfunction. The amount of silicone oil in this region is carefully adjusted by wicking any excess oil with a paper towel or a clean-room towel. As the oil gets wicked into the towel through capillary action, the perimeter of the oil droplet shrinks in size. The oil droplet diameter is adjusted such that its perimeter slightly overlaps with the co-planar electrodes on the surface. Overlap of the SLID shutter with the co-planar electrodes is an essential requirement for successful SLID shutter actuation. Without overlap, there is no change in shape or spatial position of the SLID shutter when the co-planar electrodes are actuated.

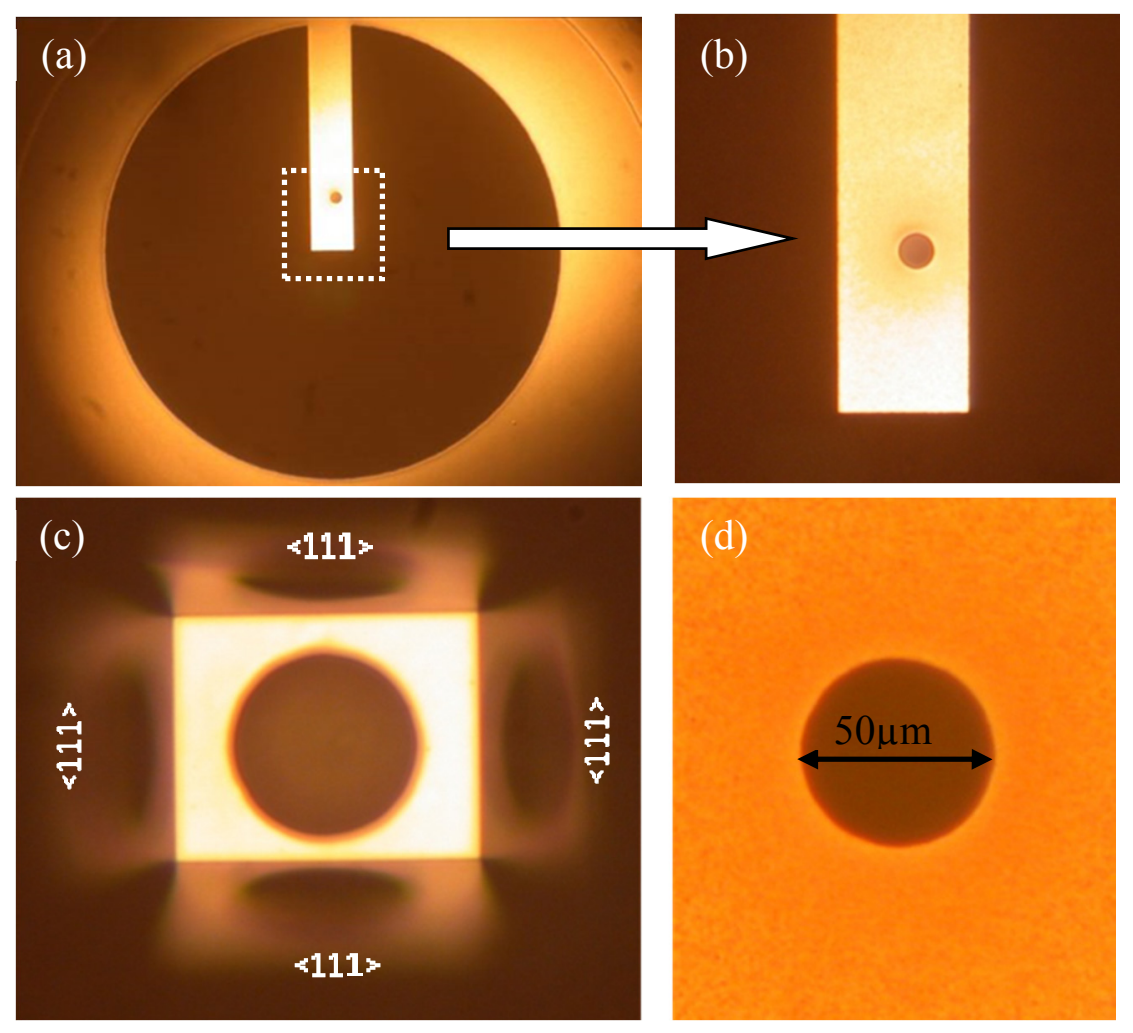

Figure 41. Images of different areas on the fabricated device (ejector): (a) Circular defectridden region with the flat bare strip containing the nozzle. (b) Close-up of (a) around the nozzle. (c) View of the nozzle from the back; $\mathrm{KOH}$ etched side-walls $\langle 111\rangle$ can be seen and (d) Close-up of nozzle from the front. 
As can be seen in Figure 41a, a circular region having a diameter of $2 \mathrm{~mm}$ on the underlying SU8 is exposed during the patterning step while all the other areas have been masked with Al. This circular regions contains a thin bare strip overlapping the circular $50 \mu \mathrm{m}$ nozzle. This bare strip is masked with $\mathrm{Al}$ during the formation of defects in the exposed circular region and hence remains free of defects. Figure $41 \mathrm{~b}$, is a zoom-in of Figure 41a. It highlights the metal coated bare strip which overlaps the nozzle. Figure 41c, is the backside view of the $\mathrm{KOH}$ etched silicon nitride wafer. This profile is similar to the $\mathrm{KOH}$ etched silicon nitride wafer that was previously used with the top cover-slip. The reflected image of the nozzle on the four sidewalls depict the smooth mirror like etch profile of the $<111>$ planes in silicon. Figure $41 \mathrm{~d}$, is the close up of $\mathrm{b}$ and depicts the circular profile of the nozzle from the top.

\subsection{Bio-printing setup}

For the bio-printing of hydrogels using the new clog-free ejector prototype, all hydrogel pre-gel solutions were prepared at $0.25 \%(\mathrm{wt} \%)$ with water. A few micro moles of a fluorescent dye were added to the solvents to help in the imaging of the ejected droplets at a later time. These pre-gel solutions were then filtered using $2 \mu \mathrm{m}$ pore size syringe filters and were then stored in airtight vials in the refrigerator until deployment in the ejectors. The extremely low concentrations prevented any cross-linking of the hydrogels during storage and the solutions remained clear at all times. For printing purposes, bare glass substrates were first cleaned using acid treatments, washed, blow dried, annealed and then oxygen plasma cleaned. They were then masked with plastic films. Slits of widths between $250 \mu \mathrm{m}$ and $500 \mu \mathrm{m}$ were manually cut into these films using a scalpel. These slits were used as stencils for the subsequent ejection of hydrogels on the glass substrates. Deposition 
of the hydrogel pre-gel liquids was carried out using the ejector equipped with the SLID shutter. Before the bio-printing, the ejectors were loaded with the pre-gel liquids and tested for satellite free ejection using the CCD microscope and strobing LED setup. The precleaned glass substrates with the attached stencils were then placed on a movable X-Y stage such that either one of the stage axis is aligned with the stencil slits on the glass substrates. The same stage axis is then adjusted such that printing starts at the extreme end of the slits. The SLID shutter is then actuated to the open position and the ejector is then turned on. Dummy runs were initially carried out on plain glass slides to get the approximate range of stage movement speeds for continuous lines of ejected droplets.

\subsection{Results and Discussion}

\subsubsection{Testing of the SLID shutter mechanism}

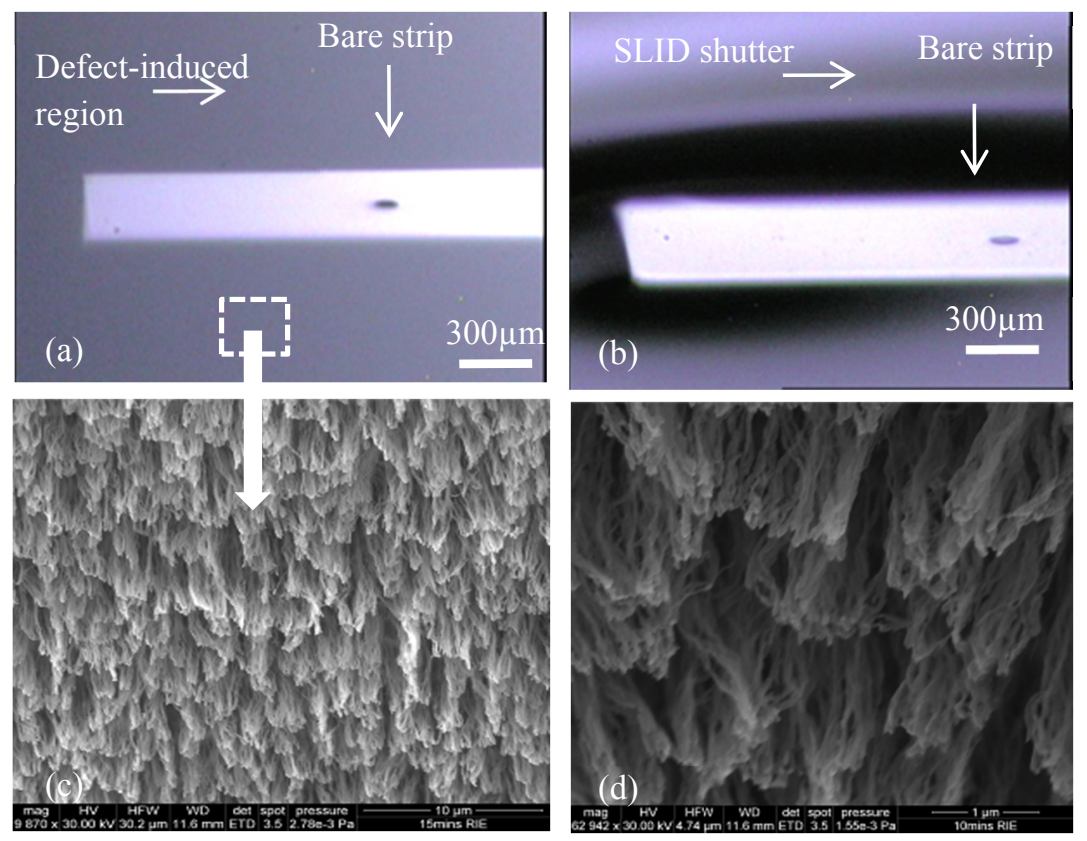

Figure 42. (a) Area around the nozzle free of defects (bare strip) in the absence of the SLID shutter material. (b) The SLID shutter material (silicone oil) while in the 'open' state pinned on the surface of the modified region but off the bare strip. (c, d) SEM images of the modified region formed by reactive Ion etching (RIE) in an oxygen environment. This modified region is composed of surface defects that are highly oleophilic. 
To better understand the working mechanism of the SLID shutter, it's important to understand the role of the flat bare strip overlapping the nozzle. The role of the modified / defect-induced region is better understood from Figure 42. As can be seen in Figure 42a, the bare strip is flat and free of defects. The roughness parameter $r$ in this region has a value of unity indicating a perfectly flat surface. Being entirely flat, this region prevents the triple-phase contact line (TCL) of the SLID shutter material from pinning onto any part of it as seen in Figure 42b. This in turn allows the TCL segment to traverse freely along this strip during actuation and de-actuation of the SLID shutter and stay free of any SLID shutter material. Furthermore, during the actuation of the SLID shutter onto the co-planar electrodes, the modified / defect-induced area remains wetted by the SLID shutter material at all times as seen in Figure 42b. Figure 42c and Figure 42d are SEM images of the modified / defect-induced areas that have a high affinity to low surface tension liquids such as the SLID shutter material.

(a)

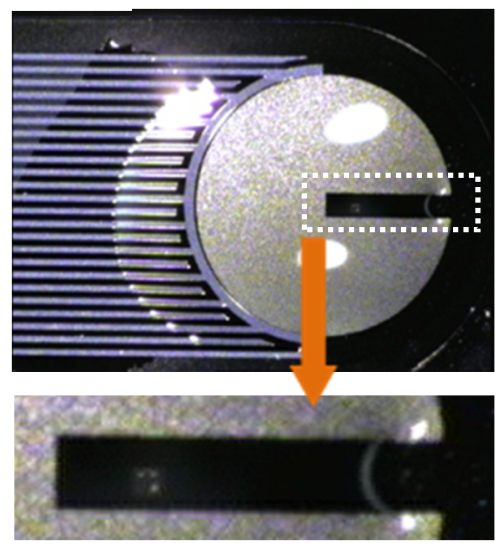

Nozzle state: OPEN (b)

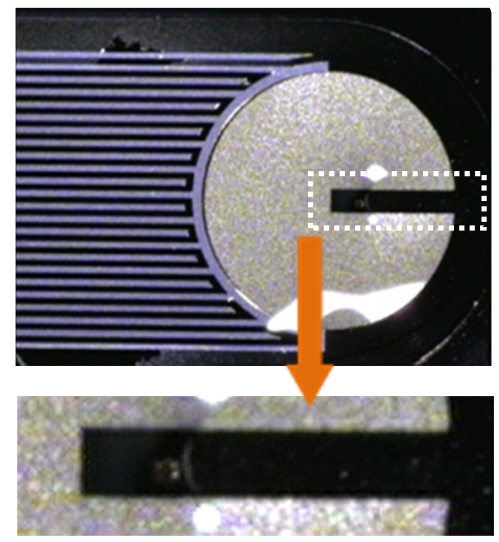

Nozzle state: OPEN (c)

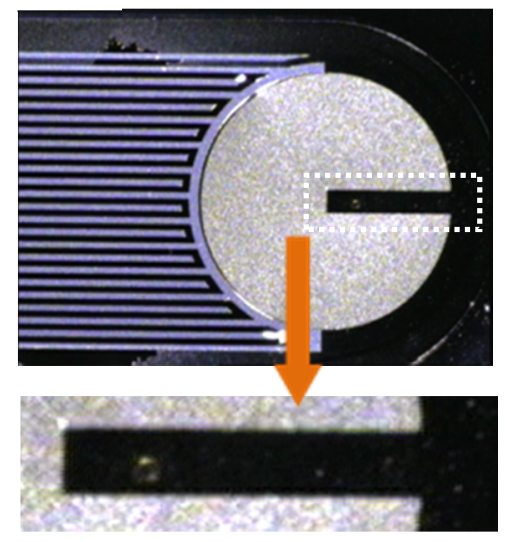

Nozzle state: CLOSED

Figure 43. Top view of the SLID shutter in the (a) closed state (b) actuated state while about to open the nozzle and (c) open state with the nozzle fully exposed. The insets below (a), (b) and (c) are the respective zoomed-in areas of the bare strip. These insets depict the position of the TCL segment that plays a key role in the nozzle opening and closing. 
The SLID shutter is actuated to the open position / state by applying a DC voltage between the pair of comb-shaped co-planar electrodes. Actuation of the SLID shutter from the closed to the open state is carried out at a voltage of 300-400 VDC. As soon as the voltage is applied, the SLID shutter elongates from an equilibrium almost-circular shaped profile and spreads along the co-planar electrodes as seen in Figure 44a-e. During this time, a segment of the TCL retreats along the bare strip while opening the nozzle as seen in Figure $43 \mathrm{~b}$ and Figure $43 \mathrm{c}$. It was observed that the movement of the TCL segment along the bare strip is a function of the applied voltage during the opening of the SLID shutter. Furthermore, it was observed that the opening of the SLID shutter and exposing of the nozzle takes less than 2 seconds. Closing the SLID shutter and thus covering the nozzle is accomplished simply by turning off the actuation voltage as seen in Figure 44f-j. Immediately after the actuation voltage is turned off, the SLID shutter restores its original circular shaped profile. Closing of the SLID shutter and covering of the nozzle takes place within a similar time period of 2 seconds. Repeatability of the SLID shutter was further tested by continuously opening and closing it. No change in behavior was observed during each cycle and all the cycles were identical. Furthermore, even after extended periods of time such as a few weeks, the behavior of the SLID shutter did not change. Also its size did not change due to fluid loss through evaporation. 

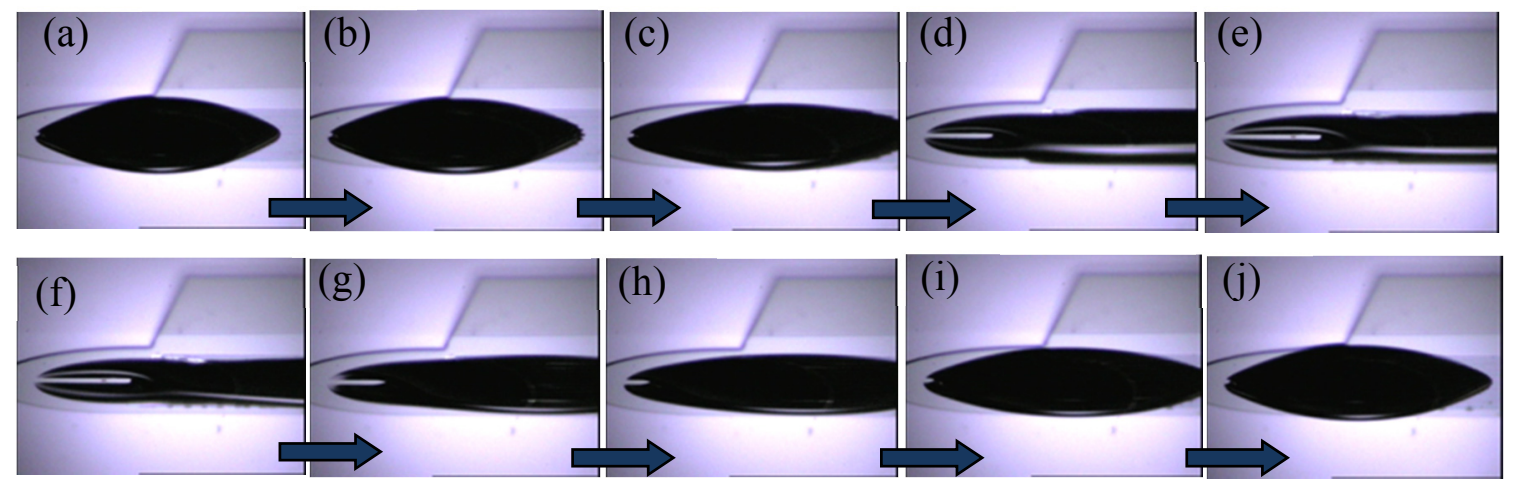

Figure 44. (a-e) SLID shutter actuated from the 'closed' state to the 'open' state with an actuation voltage of $300 \mathrm{VDC}$. $(f-j)$ SLID shutter being automatically restored from the 'open' state to the closed state by turning off the actuation voltage.

As can be seen in Figure 45a-c, de-ionized water is being ejected from the ejector. Before loading of the SLID shutter material (silicone oil) onto the modified / defect-induced region, ejection is carried out to determine all the ejection parameters that result in satellite free ejection of the bio-fluids. As soon as this is done, the SLID shutter is loaded onto the circular modified / defect-induced region and is made ready for further testing.
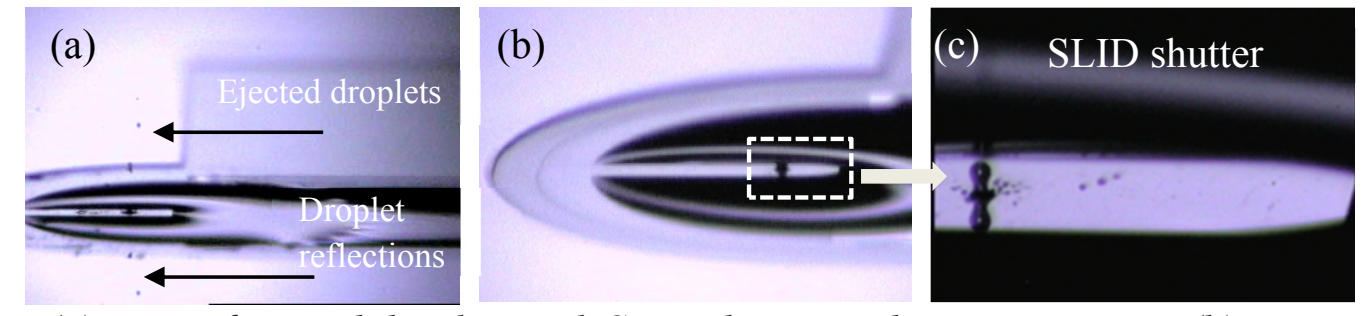

Figure 45. (a) View of ejected droplets with SLID shutter in the open position. (b) Zoomin of the circular region and bare strip around the nozzle. (c) Maximum zoom-in of the ejector nozzle.

\subsubsection{Printing of bio-fluids including proteins and hydrogels}

Droplet ejectors used as arrayers have been known to clog through a process of matrix crystallization at the nozzle. Crystallization occurs when the matrix / protein solution gets supersaturated. However, matrix crystallization was seen to be no longer a problem with the addition of a small amount of solvent to the matrix solution in the dispenser [89]. It was 
observed that the addition of 5\% isopropanol alcohol (IPA) to the matrix solution prevented its crystallization at the nozzle region by slowing solvent evaporation. Although, this development was seen as a major improvement since it enabled a reproducible addition of matrix solution to the nano-vials in sub-microliter quantities, its effects on the denaturation of proteins has yet to be evaluated.

A more effective method for the prevention of protein or matrix crystallization at the ejector nozzle, would be to prevent the solution from reaching its super saturation state. This can be done by blocking any fluidic loss through evaporation, thereby maintaining a constant solution concentration. And the SLID shutter does precisely this while positioned above the nozzle. While not in use, the SLID shutter prevents evaporation induced fluidic loss and associated changes in solution concentration. To test this technique, the ejection of several protein solutions, shown in Table VI, was carried out in ejectors integrated with the SLID shutter, between random time durations. Stable ejection was achieved without any crystallization failures at the nozzle between consecutive runs separated by up to a period of a week. However, the ejection quality varied between protein groups. Since proteins can either have globular or fibrous structures, it was observed that the ejection characteristics were protein dependent. To be more precise, it was observed that the ejection of globular protein solutions as compared to fibrous ones seemed easier. These tests prove that the SLID shutter technology can be used as an effective means in preventing protein crystallization at the nozzle. 
Table VI. Protein solutions tested in the microfluidic ejector equipped with the liquid-film SLID shutter.

\begin{tabular}{|c|c|c|c|c|}
\hline & Time before $t$ & tal nozzle failure & & \\
\hline $\begin{array}{l}\text { Biological } \\
\text { Fluids }\end{array}$ & Concentration & $\begin{array}{l}\text { SLID shutter bio } \\
\text { compatibility }\end{array}$ & $\begin{array}{c}\text { Ejection in } \\
\text { droplet } \\
\text { ejector }\end{array}$ & Comments \\
\hline Streptavidin & $100 \mu . \mathrm{gm} / \mathrm{ml}$ & $\checkmark$ & $\checkmark$ & $\begin{array}{l}\text { Stable } \\
\text { ejection }\end{array}$ \\
\hline $\begin{array}{c}\text { Creatine } \\
\text { Phosphokinase }\end{array}$ & $100 \mu . \mathrm{gm} / \mathrm{ml}$ & $\checkmark$ & $x$ & Very unstable \\
\hline Collagenase & $1 \mathrm{~m} . \mathrm{gm} / \mathrm{ml}$ & $\checkmark$ & $\checkmark$ & $\begin{array}{l}\text { Not so stable } \\
\text { ejection }\end{array}$ \\
\hline Urease & $100 \mu . \mathrm{gm} / \mathrm{ml}$ & $\checkmark$ & $\checkmark$ & $\begin{array}{c}\text { Stable } \\
\text { ejection }\end{array}$ \\
\hline Arginase & $100 \mu . \mathrm{gm} / \mathrm{ml}$ & $\checkmark$ & $\checkmark$ & $\begin{array}{c}\text { Stable } \\
\text { ejection }\end{array}$ \\
\hline Avidin & $100 \mu . \mathrm{gm} / \mathrm{ml}$ & $\checkmark$ & $\checkmark$ & $\begin{array}{c}\text { Stable } \\
\text { ejection }\end{array}$ \\
\hline Cellulase & $100 \mu . \mathrm{gm} / \mathrm{ml}$ & $\checkmark$ & $\checkmark$ & $\begin{array}{c}\text { Not so stable } \\
\text { ejection }\end{array}$ \\
\hline
\end{tabular}

Tissue engineering (TE) also employs bio-printing of scaffolding hydrogel solutions deposited layer by layer while seeding cells either simultaneously or between consecutive runs. As stated earlier, a common problem is that nozzle-based ejectors used for tissue printing are prone to clogging due to fast evaporation and accumulation of the bio-fluid and biomaterials at the nozzle tip. In the case of hydrogels for example, clogging is caused when the polymers in the aqueous solutions begin to crosslink and transform into hydrogel networks when solvent evaporation occurs. This can be a costly affair due to the printing equipment downtime each time clogging occurs especially when the tissue is half way printed. In our research, we have used various hydrogel solutions to demonstrate how some 
of the problems associated with these hydrogels in tissue-engineering (TE) applications can be overcome, using the SLID shutter technology.

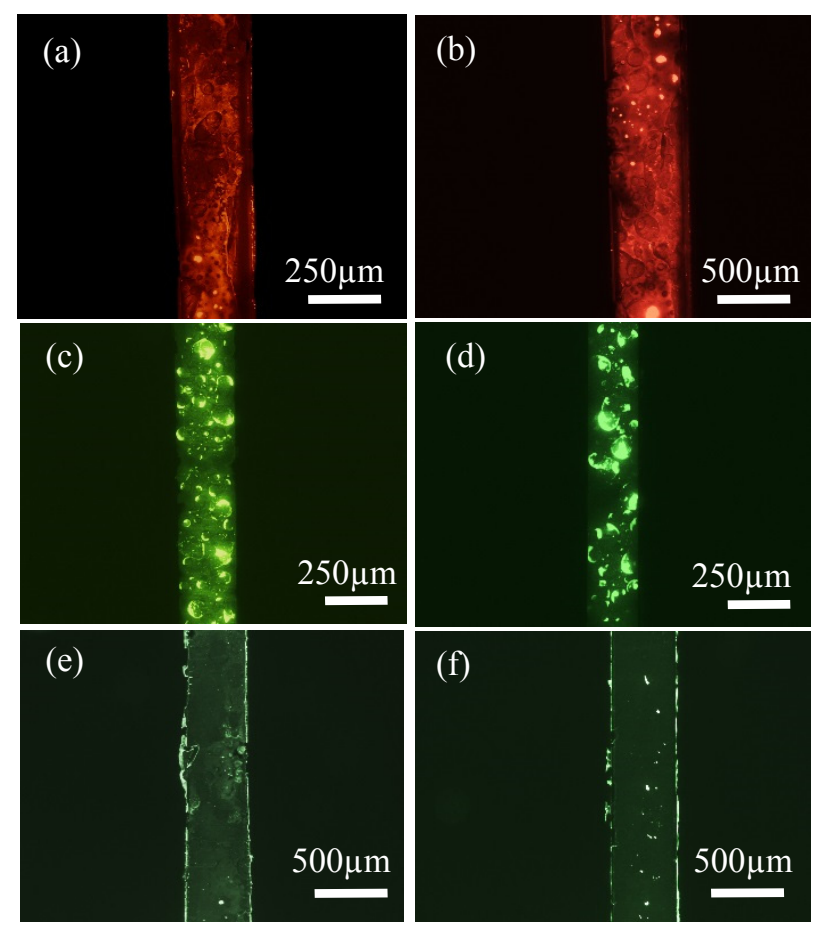

Figure 46. Fluorescence microscopy images of hydrogels printed on bare glass substrates. $(a, b)$ printed Gelatin stained with Rhodamine B dye. (c, d) printed Agarose stained with Acridin Orange dye. $(e, f)$ - printed Collagen stained with Fluorescein dye.

One of the first hydrogels tested in the ejector equipped with the SLID shutter was Collagen. Collagen gels have been widely utilized for reconstruction of liver, skin, blood vessel, and small intestine. Collagen has been used as a tissue culture scaffold or artificial skin due to the ready attachment of many different cell types and its cell-based degradation. It is the most widely used tissue-derived natural polymer and it is a main component of extracellular matrices of mammalian tissues including skin, bone, cartilage, tendon, and ligament. Collagen meets many of the biological design parameters, as it is composed of specific combinations of amino acid sequences that are recognized by cells and degraded by enzymes secreted from the cells (i.e. collagenase). The second hydrogel tested in the clog-free ejectors was Gelatin. Gelatin is a derivative of collagen, formed by breaking the 
natural triple-helix structure of collagen into single-strand molecules. There are two types of gelatin, gelatin A and gelatin B. Gelatin A is prepared by acidic treatment before thermal denaturation, while gelatin $\mathrm{B}$ is processed by alkaline treatment that leads to a high carboxylic content. Gelatin easily forms gels by changing the temperature of its solution. Similar to Collagen, it has been used in many tissue engineering applications due to its biocompatibility and ease of gelation. Gelatin gels have also been utilized for delivery of growth factors to promote vascularization of engineered new tissue. Yet another hydrogel which has been utilized in TE applications has been Agarose which was tested in the ejectors. These hydrogels were ejected at $24 \mathrm{hr}$ intervals with the SLID shutter capping the nozzle between tests, as seen in Figure 46. It was observed that the nozzles ejecting these hydrogels clogged within a few hours while the ejectors were not in use with the SLID shutter left open. However, a good quality of ejection was still achievable when the nozzle was closed and hermetically sealed with the SLID shutter even after a period of 15 days for one of the hydrogel solutions. These results have been summarized in Table VII. This technique can be extended to a wide range of micro-engineered hydrogels that are currently being used in tissue printing. 
Table VII. Evaporation speed data for various hydrogel solutions when the SLID shutter is opened or closed.

\begin{tabular}{|c|c|c|}
\hline & \multicolumn{2}{|c|}{ Time before total nozzle failure } \\
\hline $\begin{array}{c}\text { Biological } \\
\text { Fluids }\end{array}$ & w/o SLID shutter & With the SLID shutter \\
\hline DI water & $6 \mathrm{hr}$ & $>3$ weeks \\
\hline Collagen & $2-3 \mathrm{hr}$ & 4 \\
\hline Agarose & $2-3 \mathrm{hr}$ & 9 days \\
\hline Gelatin & $2-3 \mathrm{hr}$ & 15 days \\
\hline
\end{tabular}

The actual mechanism of clogging is not understood at this point but is believed to be due to evaporation induced coagulation and cross-linking of the hydrogel solutions which occurs within a span of a few hours. These results prove that this technique can be extended to a wide range of micro-engineered hydrogels that are currently being used in tissue printing.

Certain biological entities such as nucleosides that are preserved in non-aqueous or partly aqueous liquids have also been tested in the clog-free ejector. Specifically, nucleoside solutions in de-ionized water/Acetonitrile were tested to determine the efficacy of the ‘Sliding Liquid Drop’ (SLID) shutter technology. In a simple test, 2\% (wt\%) Adenosine in a Acetonitrile/water (1:1) solution was tested in the ejectors. Stable ejection was achieved as expected, however long term clog-free testing could not be completed as the solution affected the epoxy used in the device bonding step. In less dilute solutions of water/Acetonitrile (2:1) with the nucleosides, stable ejections could be achieved even after a week of testing. One interesting observation was the crystallization of the nucleoside 
solution upon being ejected on the substrate within a relatively short period. This is the same mechanism that causes nozzle failures. As can be seen in Figure 47, optical microscope images do not reveal any clusters of crystallized Adenosine. However, when the same sample was viewed under fluorescence microscopy, crystallized agglomerates of Adenosine could be observed. It is these crystallized agglomerates that induce nozzle clogging in droplet ejectors when bio-fluid evaporation at the nozzle is not kept in check. These results prove that the detrimental effects of crystallization which pose a problem to the nozzles of droplet ejectors and inkjet print heads could be circumvented with the use of the SLID shutter.
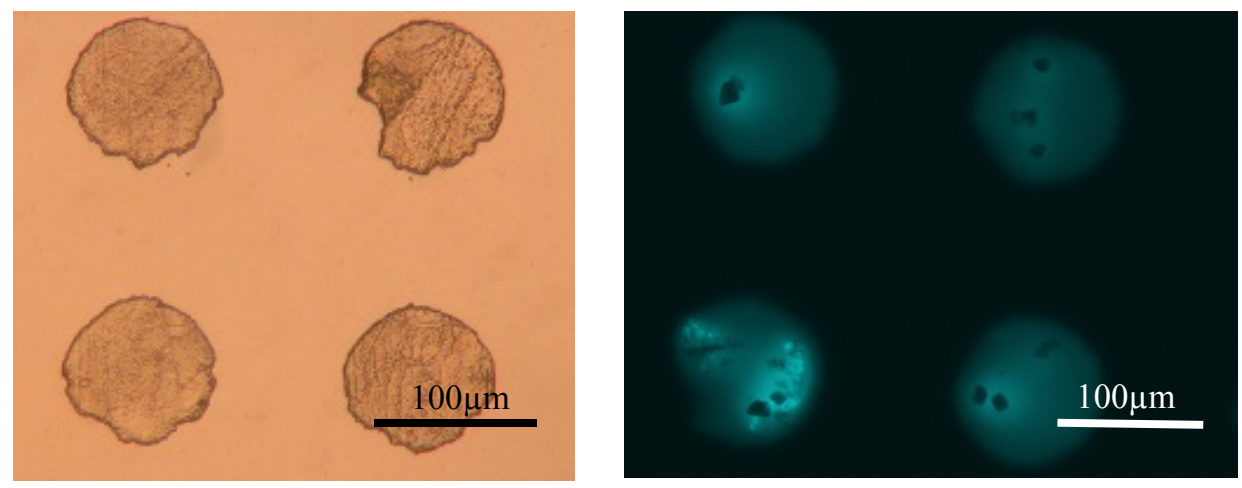

Figure 47. Optical microscopic images of Adenosine ejected on bare glass substrates. Fluorescence images of the same substrate which reveal crystallized agglomerates formed during solvent evaporation upon ejection.

Reactive bio printing has commonly been used for the purification or detection of various bio-molecules. In order to demonstrate how the ejectors equipped with the SLID shutter technology can be used for these applications, a simple test was carried out. In this test, a 1\% (wt $\%$ ) Adenosine solution (1:2 Acetonitrile : water) was ejected onto patterned Parylene AM features. Parylene AM has one methylene amine per repeat group and an abundance of $-\mathrm{NH}_{2}$ groups on the surface. This test was carried out to observe specific binding of bio molecules to areas of interest. However, ejection was carried out only on 
some of the patterned features. As can be seen in Figure 48, Adenosine was seen to bind only to the reactive areas that possessed $-\mathrm{NH}_{2}$ surface groups. To observe this specific binding, the samples were immersed in a vial of Acridine orange, immediately after washing off any excess ejected Adinosine on the features. When viewed under the fluorescence microscope, it was observed that the dye only attached to the Adinosine tagged Parylene AM features.

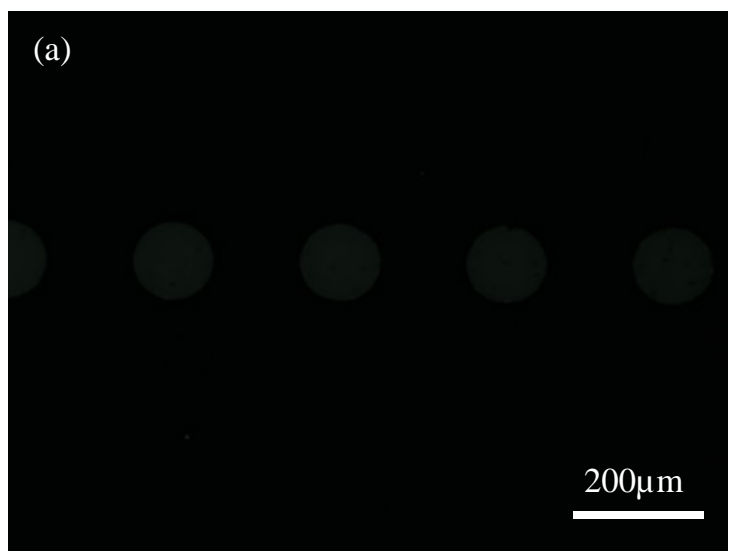

Figure 48. Fluorescence microscopic images of Adenosine ejected on patterned Parylene AM on glass substrates. (a) Adenosine ejected on none of the patterned Parylene AM features. (b) Adenosine ejected only on some of the patterned Parylene AM features to the right.

These tests prove that this technology has the potential for De novo oligonucleotide synthesis using inkjet technologies. These tests prove that the SLID shutter technology makes possible the reliable usage of standard phosphoramidite chemistry for oligoarray synthesis which allows longer oligonucleotide reporters to be synthesized. Since the last few years, oligo arrays on which oligos are synthesized have been using inkjet technologies for their synthesis.

In summary, the SLID shutter mechanism integrated over a droplet ejector enabled the ejection of volatile bio-fluids without any clogging related issues, over long periods of time. This nozzle capping mechanism has immense application in tissue engineering where 
inkjet printheads in bio-printers are used for the generation of in-vitro tissues as well as organs. Furthermore, this nozzle capping mechanism automatically shuts the nozzle after printing and utilizes a material that is bio friendly to almost all biological based fluids. Using the clog-free ejector implemented with the SLID shutter technology, bio printing of several biological fluids was accomplished. Hydrogels, protein solutions as well as DNA base components were successfully printed using this technique over long periods of time. More specifically, the potential of this technology - automatic nozzle capping with the SLID shutter - for application in Proteomics and tissue engineering have been highlighted in this chapter. 


\section{Chapter 6}

\section{FUTURE RESEARCH AND CONCLUSION}

A microfluidic valve based on an immiscible and non-volatile liquid film as a valve-core has been developed and used to successfully prevent evaporation of working fluids through microscopic ports. Many immiscible fluids to various aqueous solutions were tested to determine the most suitable candidate valve-core material. By moving the thin liquid film to cover or uncover a tiny microfluidic port with a liquid-air interface, microscopic evaporation through the port has been very well controlled. It has been clearly shown that the fluidic loss of the working fluid by quick evaporation as well as clogging due to residue left by evaporation, can be stopped by employing the LiFE microfluidic valve. We believe that this finding has immense potential in the prevention of clogging problems in various microfluidic systems having air-exposed microfluidic ports.

We have also shown how low surface tension liquids can be confined along pre-defined microfluidic paths using oil-repellent surfaces. This is the first time wherein confinement of low surface tension liquids along microfluidic paths has been demonstrated. By understanding the liquid repellent characteristics of superoleophobic surfaces, those of the oil-repellent surfaces were designed. The oil-repellent surfaces were initially envisioned to be used on the bio- ejector surface because of its many advantages such as guiding of the microfluidic valve in top-covered as well as open-channel devices. Furthermore, the discovery of virtual walls for supporting low surface tension liquids in a microchannel was a direct outcome while developing robust oil-repellent surfaces, which has been described in great detail in this report. The oil-repellent surfaces have a lot of potential for use in Lab-on-a-chip (LOC) devices and other $\mu$-TAS systems. For example they could be used 
for the separation of laminar flow oil / water emulsions in microfluidic chips. One study that would be worth carrying out is understanding the liquid repellent characteristics of the micromachined surfaces when the dimensions of the features are scaled down to the nano scale. One important property that may appear to be superior at such scales is the optical transparency of these surfaces. At the micro scale, scattering of light due to the micromachined features causes light to disperse reducing the transparency of the surface. As of now, we have not envisioned any applications where the transparency of these liquidrepellent surface is crucial. One more interesting application where these oil-repellent surfaces could be used, is for promoting cell adhesion. The dimensions of these micromachined features is comparable to those of living cells. Moreover, the micromachined features could help cells to anchor onto the re-entrant curvatures. Lastly but most importantly, virtual walls using oil-repellent surfaces could have immense potential in Lab-on-a-chip (LOC) devices catered to the petrochemical industry. Performing various analysis of crude petroleum samples on-site using LOC devices would be helpful to those working in the field. However, one difficulty in analyzing petroleum products especially crude oil, has been the different fractions of hydrocarbon components present. Moreover, some of these components are highly volatile and pose a problem in LOC devices. This is where oil-repellent surfaces could benefit LOC devices targeted to the petrochemical industry for on-site sample analysis. With the absence of physical walls, liquid streams of crude oil could be channeled to the different reaction zones on the LOC device while the volatile components could escape from the 'invisible' sidewalls. In comparison, the presence of physical walls could hinder the flow of crude oil streams as the volatile components could vaporize resulting in trapped pockets on gas in the channel. 
Furthermore, commonly used elastomers such as PDMS, used as sidewalls, could be adversely affected by swelling in the presence of petroleum products. Another advantage in using oil-repellent surfaces for these types of applications is the tuning of the oilrepellency effect. We believe that by varying the micromachined feature dimensions, oils with varying surface tensions could be handled more efficiently, especially for oil mixtures such as crude oil. Often, speculation may not be the right approach and experimentation would need to be done to validate these claims.

The 'slippery' surfaces were another type of surface that was discovered while trying to develop surfaces that would exhibit minimal drag to the microfluidic valve on the ejector surface. As earlier mentioned, this was done with the intent of reducing the microfluidic valve driving voltage on the ejector surface. Since surfaces composed of solid and air fractions - also called composite surfaces - cause liquid droplets to roll off easily, our first choice was to use these surfaces on the ejector surface. However, the high Laplace pressure within the microfluidic valve, inside top covered devices, do not permit the use of composite surfaces in these devices. Composite surfaces composed of solid and air fractions cause the microfluidic valve to pin strongly on them, thereby increasing driving voltages. However when the composite surfaces were modified from solid-air fractions to solid-liquid fractions by using a lubricating liquid, the microfluidic valve could easily glide across these surfaces. And thus 'slippery' surfaces were synthesized. At the same time when slippery surfaces were discovered by us, it was discovered elsewhere. Because of that, we went a step further and decided to embed the driving mechanism into these slippery surfaces; this technique had never been demonstrated before. Using this technique, the actuation of the microfluidic valve along the ejector surface using L-DEP was 
demonstrated. Although this surface was very 'slippery' to the microfluidic valve as well as other liquids, its implementation could not be realized. This was due to the fact that the lubricating liquid which was used on the slippery surfaces, have the potential of draining into the ejector nozzle because of its extremely low surface tension.

The discovery of slippery surfaces has a lot of utility in microfluidic systems where liquids need to be transported in closed channels across compartments in fluidic chips. By rendering the inner walls of microfluidic channels slippery, microfluids could be transported more easily using a lower actuation power. However the actual implementation of this scheme is much more complex from the fabrication standpoint. For example, we have only succeeded in developing slippery surfaces on planar substrates and not on 3D features. Nevertheless, these surfaces open up the possibility of being adapted to various other microfluidic components other than microfluidic channels.

Finally, we have demonstrated the working of a normally-closed 'Sliding liquid Drop' (SLID) shutter for droplet ejectors by making use of nanostructured surfaces. The nanostructures aid in the SLID shutter attaining its normally-closed nature. This microfluidic shutter has a lot of advantages over the previous microfluidic valve that we had demonstrated. Some of these advantages include faster response times, the automatic closing mechanism, cheaper cost of fabrication as well as simpler device assembly. With this new shutter implementation, we have successfully demonstrated well-ordered printing of biological fluids such as hydrogels. The faster response times enable it to be used for the ejection of highly volatile liquids such as Acetonitrile used for oligonucleotide synthesis. Furthermore, the nanostructures used on the surface improve the anchoring of the SLID shutter to the nozzle and provide a strong nozzle capping mechanism. This 
improves the durability of the SLID shutter making it resistant to mechanical shocks and vibrations. Previously, the sandwiched microfluidic valve did not possess this level of durability and suffered from a lot of other problems. The successful printing of several hydrogels, used for tissue engineering applications (TE), as well as certain protein groups for various other applications has been demonstrated. We believe this technique can also be used for the printing of various other fluids. For example, printing of 'electronic inks'conductive or semi-conductive fluids - on various substrates can be achieved using this technology - the SLID shutter. Electronic inks are very expensive, costing hundreds of dollars for a few milligrams. And this is why protecting these inks from the detrimental effects of evaporation and oxidation is crucial. In a similar manner, nanoparticle solutions that are used for the fabrication of microstructures on flat substrates can be well protected inside the printhead.

In summary, this work demonstrates how the area of microfluidics can be facilitated by the various discoveries. New fluidic components, new surface types as well as new liquid handling techniques highlighted in this study can be valuable tools to researchers working in similar disciplines. 


\section{REFERENCES}

[1] H. F. Bohn and W. Federle, "Insect aquaplaning: Nepenthes pitcher plants capture prey with the peristome, a fully wettable water-lubricated anisotropic surface," Proceedings of the National Academy of Sciences of the United States of America, vol. 101, pp. 14138-14143, 2004.

[2] W. B. Zimmerman, Microfluidics: history, theory and applications vol. 466: Springer, 2006.

[3] F. P. Incropera, A. S. Lavine, and D. P. DeWitt, Fundamentals of heat and mass transfer: John Wiley \& Sons Incorporated, 2011.

[4] M. Nakamura, A. Kobayashi, F. Takagi, A. Watanabe, Y. Hiruma, K. Ohuchi, et al., "Biocompatible inkjet printing technique for designed seeding of individual living cells," Tissue engineering, vol. 11, pp. 1658-1666, 2005.

[5] C. Herbert and R. Johnstone, Mass spectrometry basics: CRC, 2002.

[6] V. Mironov, R. Markwald, and G. Forgacs, "ORGAN PRINTING: SELFASSEMBLING CELL AGGREGATES AS" BIOINK"," Science and Medicine, vol. 9, pp. 69-71, 2003.

[7] S. Magdassi and M. Moshe, "Patterning of organic nanoparticles by ink-jet printing of microemulsions," Langmuir, vol. 19, pp. 939-942, 2003.

[8] A. Feriani and J. Hess, "VOLATILE LIQUID DROPLET DISPENSER DEVICE," ed: Google Patents, 2009.

[9] E. Litborn and J. Roeraade, "Liquid lid for biochemical reactions in chip-based nanovials," Journal of Chromatography B: Biomedical Sciences and Applications, vol. 745, pp. 137-147, 2000.

[10] E. Berthier, J. Warrick, H. Yu, and D. Beebe, "Managing evaporation for more robust microscale assays Part 1. Volume loss in high throughput assays," Lab on a Chip, vol. 8, p. 852, 2008.

[11] E. Litborn, Å. Emmer, and J. Roeraade, "Chip-based nanovials for tryptic digest and capillary electrophoresis* 1," Analytica Chimica Acta, vol. 401, pp. 11-19, 1999.

[12] E. Litborn, Å. Emmer, and J. Roeraade, "Parallel reactions in open chip-based nanovials with continuous compensation for solvent evaporation,"

Electrophoresis, vol. 21, pp. 91-99, 1999.

[13] G. Mayer and J. Köhler, "Micromechanical compartments for biotechnological applications: fabrication and investigation of liquid evaporation," Sensors and Actuators A: Physical, vol. 60, pp. 202-207, 1997.

[14] L. S. Fan, Y. C. Tai, and R. S. Muller, "IC-processed electrostatic micromotors," Sensors and Actuators, vol. 20, pp. 41-47, 1989.

[15] X. C. Man, N. R. Manwell, and J. L. Burkman, "Fiber cleaning system for inkjet printhead wipers," ed: Google Patents, 1999.

[16] A. Lakhtakia and R. J. Martin-Palma, Engineered Biomimicry: Newnes, 2013.

[17] W. Barthlott and C. Neinhuis, "Purity of the sacred lotus, or escape from contamination in biological surfaces," Planta, vol. 202, pp. 1-8, 1997.

[18] K. Koch and W. Barthlott, "Superhydrophobic and superhydrophilic plant surfaces: an inspiration for biomimetic materials," Philosophical Transactions of 
the Royal Society A: Mathematical, Physical and Engineering Sciences, vol. 367, pp. 1487-1509, 2009.

[19] K. Koch, B. Bhushan, Y. C. Jung, and W. Barthlott, "Fabrication of artificial Lotus leaves and significance of hierarchical structure for superhydrophobicity and low adhesion," Soft Matter, vol. 5, pp. 1386-1393, 2009.

[20] F. Iwagami, "Ink jet printer nozzle clogging-preventive device," ed: Google Patents, 1990.

[21] S. K. Cho, H. Moon, and C. J. Kim, "Creating, transporting, cutting, and merging liquid droplets by electrowetting-based actuation for digital microfluidic circuits," Microelectromechanical Systems, Journal of, vol. 12, pp. 70-80, 2003.

[22] U. C. Yi and C. J. Kim, "Soft printing of droplets pre-metered by electrowetting," Sensors and Actuators A: Physical, vol. 114, pp. 347-354, 2004.

[23] N. Kumari, V. Bahadur, and S. Garimella, "Electrical actuation of dielectric droplets," Journal of Micromechanics and Microengineering, vol. 18, p. 085018, 2008.

[24] M. L. Minges, Electronic Materials Handbook: Packaging: CRC, 1989.

[25] C. Willert, B. Stasicki, J. Klinner, and S. Moessner, "Pulsed operation of highpower light emitting diodes for imaging flow velocimetry," Measurement Science and Technology, vol. 21, p. 075402, 2010.

[26] H. Zhao, K. Y. Law, and V. Sambhy, "Fabrication, surface properties, and origin of superoleophobicity for a model textured surface," Langmuir, vol. 27, pp. 59275935, 2011.

[27] H. Zhao and K. Y. Law, "Directional Self Cleaning Superoleophobic Surface," Langmuir, 2012.

[28] A. Tuteja, W. Choi, M. Ma, J. M. Mabry, S. A. Mazzella, G. C. Rutledge, et al., "Designing superoleophobic surfaces," Science, vol. 318, pp. 1618-1622, 2007.

[29] A. Tuteja, W. Choi, J. M. Mabry, G. H. McKinley, and R. E. Cohen, "Robust omniphobic surfaces," Proceedings of the National Academy of Sciences, vol. 105, p. 18200, 2008.

[30] T. Wu and Y. Suzuki, "Design, Microfabrication and Evaluation of Robust HighPerformance Superlyophobic Surfaces," Sensors and Actuators B: Chemical, 2011.

[31] W. Choi, A. Tuteja, J. M. Mabry, R. E. Cohen, and G. H. McKinley, "A modified Cassie-Baxter relationship to explain contact angle hysteresis and anisotropy on non-wetting textured surfaces," Journal of colloid and interface science, vol. 339, pp. 208-216, 2009.

[32] C. Ding, Y. Zhu, M. Liu, L. Feng, M. Wan, and L. Jiang, "PANI nanowire film with underwater superoleophobicity and potential-modulated tunable adhesion for no loss oil droplet transport," Soft Matter, 2012.

[33] V. Jokinen, L. Sainiemi, and S. Franssila, "Controlled Lateral Spreading and Pinning of Oil Droplets Based on Topography and Chemical Patterning," Langmuir, 2011.

[34] A. Ahuja, J. Taylor, V. Lifton, A. Sidorenko, T. Salamon, E. Lobaton, et al., "Nanonails: A simple geometrical approach to electrically tunable superlyophobic surfaces," Langmuir, vol. 24, pp. 9-14, 2008. 
[35] L. Hong and T. Pan, "Surface microfluidics fabricated by photopatternable superhydrophobic nanocomposite," Microfluidics and nanofluidics, vol. 10, pp. 991-997, 2011.

[36] H. H. Lai, W. Xu, and N. L. Allbritton, "Use of a virtual wall valve in polydimethylsiloxane microfluidic devices for bioanalytical applications," Biomicrofluidics, vol. 5, p. 024105, 2011.

[37] N. Verplanck, Y. Coffinier, V. Thomy, and R. Boukherroub, "Wettability switching techniques on superhydrophobic surfaces," Nanoscale Research Letters, vol. 2, pp. 577-596, 2007.

[38] V. Jokinen, L. Sainiemi, and S. Franssila, "Controlled lateral spreading and pinning of oil droplets based on topography and chemical patterning," Langmuir, vol. 27, pp. 7314-7320, 2011.

[39] T. Cubaud and M. Fermigier, "Faceted drops on heterogeneous surfaces," EPL (Europhysics Letters), vol. 55, p. 239, 2007.

[40] T. Cubaud and M. Fermigier, "Faceted drops on heterogeneous surfaces," EPL (Europhysics Letters), vol. 55, p. 239, 2001.

[41] T. Cubaud, M. Fermigier, and P. Jenffer, "Spreading of large drops on patterned surfaces," Oil \& Gas Science and Technology, vol. 56, pp. 23-31, 2001.

[42] V. S. Nikolayev, "Dynamics and depinning of the triple contact line in the presence of periodic surface defects," Journal of Physics: Condensed Matter, vol. 17, p. 2111, 2005.

[43] J. Berthier, Microdrops and Digital Microfluidics: Processing, Development, and Applications vol. 1: William Andrew Publishing, 2007.

[44] J. Ou, B. Perot, and J. P. Rothstein, "Laminar drag reduction in microchannels using ultrahydrophobic surfaces," Physics of fluids, vol. 16, p. 4635, 2004.

[45] T. Min and J. Kim, "Effects of hydrophobic surface on skin-friction drag," Physics of Fluids, vol. 16, p. L55, 2004.

[46] L. J. Chen, M. Chen, H. D. Zhou, and J. M. Chen, "Preparation of superhydrophobic surface on stainless steel," Applied Surface Science, vol. 255, pp. 3459-3462, 2008.

[47] A. Tuteja, W. Choi, G. H. McKinley, R. E. Cohen, and M. F. Rubner, "Design parameters for superhydrophobicity and superoleophobicity," MRS bulletin, vol. 33, pp. 752-758, 2008.

[48] B. Bhushan, "Biomimetics: lessons from nature-an overview," Philosophical Transactions of the Royal Society A: Mathematical, Physical and Engineering Sciences, vol. 367, pp. 1445-1486, 2009.

[49] B. Bhushan and Y. Chae Jung, "Wetting study of patterned surfaces for superhydrophobicity," Ultramicroscopy, vol. 107, pp. 1033-1041, 2007.

[50] W. BARTHLOTT, C. NEINHUIS, D. CUTLER, F. DITSCH, I. MEUSEL, I. THEISEN, et al., "Classification and terminology of plant epicuticular waxes," Botanical Journal of the Linnean Society, vol. 126, pp. 237-260, 1998.

[51] H. Y. Erbil, A. L. Demirel, Y. Avc1, and O. Mert, "Transformation of a simple plastic into a superhydrophobic surface," Science, vol. 299, pp. 1377-1380, 2003.

[52] A. Lafuma and D. Quéré, "Superhydrophobic states," Nature materials, vol. 2, pp. 457-460, 2003. 
[53] T. S. Wong, S. H. Kang, S. K. Y. Tang, E. J. Smythe, B. D. Hatton, A. Grinthal, et al., "Bioinspired self-repairing slippery surfaces with pressure-stable omniphobicity," Nature, vol. 477, pp. 443-447, 2011.

[54] M. Nosonovsky, "Materials science: Slippery when wetted," Nature, vol. 477, pp. 412-413, 2011.

[55] J. Aizenberg, "Adaptive fluid-infused porous films with tunable transparency and wettability," 2013.

[56] P. Kim, T.-S. Wong, J. Alvarenga, M. J. Kreder, W. E. Adorno-Martinez, and J. Aizenberg, "Liquid-Infused Nanostructured Surfaces with Extreme Anti-Ice and Anti-Frost Performance," ACS nano, vol. 6, pp. 6569-6577, 2012.

[57] U. Bauer, H. F. Bohn, and W. Federle, "Harmless nectar source or deadly trap: Nepenthes pitchers are activated by rain, condensation and nectar," Proceedings of the Royal Society B: Biological Sciences, vol. 275, pp. 259-265, 2008.

[58] A. K. Epstein, T.-S. Wong, R. A. Belisle, E. M. Boggs, and J. Aizenberg, "Liquid-infused structured surfaces with exceptional anti-biofouling performance," Proceedings of the National Academy of Sciences, vol. 109, pp. 13182-13187, 2012.

[59] W. Ma, Y. Higaki, H. Otsuka, and A. Takahara, "Perfluoropolyether-infused nano-texture: a versatile approach to omniphobic coatings with low hysteresis and high transparency," Chemical Communications, vol. 49, pp. 597-599, 2013.

[60] A. Lafuma and D. Quéré, "Slippery pre-suffused surfaces," EPL (Europhysics Letters), vol. 96, p. 56001, 2011.

[61] M. Rahman and A. Jacobi, "Wetting behavior and drainage of water droplets on microgrooved brass surface," 2011, p. 17.

[62] M. A. Rahman and A. M. Jacobi, "Drainage of frost melt water from vertical brass surfaces with parallel microgrooves," International Journal of Heat and Mass Transfer, 2011.

[63] O. Bliznyuk, V. Veligura, E. S. Kooij, H. J. W. Zandvliet, and B. Poelsema, "Metastable droplets on shallow-grooved hydrophobic surfaces," Physical Review $E$, vol. 83, p. 041607, 2011.

[64] Y. Chen, B. He, J. Lee, and N. A. Patankar, "Anisotropy in the wetting of rough surfaces," Journal of colloid and interface science, vol. 281, pp. 458-464, 2005.

[65] M. Morita, T. Koga, H. Otsuka, and A. Takahara, "Macroscopic-wetting anisotropy on the line-patterned surface of fluoroalkylsilane monolayers," Langmuir, vol. 21, pp. 911-918, 2005.

[66] L. Feng, S. Li, Y. Li, H. Li, L. Zhang, J. Zhai, et al., "Super-Hydrophobic Surfaces: From Natural to Artificial," Advanced materials, vol. 14, pp. 18571860, 2002.

[67] Y. Zheng, X. Gao, and L. Jiang, "Directional adhesion of superhydrophobic butterfly wings," Soft Matter, vol. 3, pp. 178-182, 2006.

[68] D. Quéré, "Wetting and roughness," Annu. Rev. Mater. Res., vol. 38, pp. 71-99, 2008.

[69] H. D. Kim and M. H. Kim, "Effect of nanoparticle deposition on capillary wicking that influences the critical heat flux in nanofluids," Applied physics letters, vol. 91, pp. 014104-014104-3, 2007. 
[70] B. He and J. Lee, "Dynamic wettability switching by surface roughness effect," 2003, pp. 120-123.

[71] T. Jones, "Liquid dielectrophoresis on the microscale," Journal of Electrostatics, vol. 51, pp. 290-299, 2001.

[72] M. S. Dhindsa, N. R. Smith, J. Heikenfeld, P. D. Rack, J. D. Fowlkes, M. J. Doktycz, et al., "Reversible electrowetting of vertically aligned superhydrophobic carbon nanofibers," Langmuir, vol. 22, pp. 9030-9034, 2006.

[73] J. D. Smith, R. Dhiman, S. Anand, E. Reza-Garduno, R. E. Cohen, G. H. McKinley, et al., "Droplet mobility on lubricant-impregnated surfaces," Soft Matter, vol. 9, pp. 1772-1780, 2013.

[74] H. Zhao and K.-Y. Law, "Directional Self-Cleaning Superoleophobic Surface," Langmuir, vol. 28, pp. 11812-11818, 2012.

[75] J. J. Marler, J. Upton, R. Langer, and J. P. Vacanti, "Transplantation of cells in matrices for tissue regeneration," Advanced drug delivery reviews, vol. 33, pp. 165-182, 1998.

[76] A. Atala, "Engineering organs," Current opinion in biotechnology, vol. 20, pp. 575-592, 2009.

[77] Y. M. Bello, A. F. Falabella, and W. H. Eaglstein, "Tissue-engineered skin," American journal of clinical dermatology, vol. 2, pp. 305-313, 2001.

[78] S. Ashiku, M. Randolph, and C. Vacanti, "Tissue engineered cartilage," in Materials Science Forum, 1997, pp. 129-150.

[79] H. Petite, V. Viateau, W. Bensaid, A. Meunier, C. de Pollak, M. Bourguignon, et al., "Tissue-engineered bone regeneration," Nature biotechnology, vol. 18, pp. 959-963, 2000.

[80] N. L'Heureux, N. Dusserre, G. Konig, B. Victor, P. Keire, T. N. Wight, et al., "Human tissue-engineered blood vessels for adult arterial revascularization," Nature medicine, vol. 12, pp. 361-365, 2006.

[81] V. Mironov, T. Boland, T. Trusk, G. Forgacs, and R. R. Markwald, "Organ printing: computer-aided jet-based 3D tissue engineering," TRENDS in Biotechnology, vol. 21, pp. 157-161, 2003.

[82] M. Nakamura, Y. Nishiyama, C. Henmi, S. Iwanaga, H. Nakagawa, K. Yamaguchi, et al., "Ink jet three-dimensional digital fabrication for biological tissue manufacturing: analysis of alginate microgel beads produced by ink jet droplets for three dimensional tissue fabrication," Journal of Imaging Science and Technology, vol. 52, p. 060201, 2008.

[83] D. Y. Wang, Y. C. Huang, H. Chiang, A. M. Wo, and Y. Y. Huang, "Microcontact printing of laminin on oxygen plasma activated substrates for the alignment and growth of Schwann cells," Journal of Biomedical Materials Research Part B: Applied Biomaterials, vol. 80, pp. 447-453, 2007.

[84] T. R. Sodunke, K. K. Turner, S. A. Caldwell, K. W. McBride, M. J. Reginato, and H. Noh, "Micropatterns of Matrigel for three-dimensional epithelial cultures," Biomaterials, vol. 28, pp. 4006-4016, 2007.

[85] P. G. Campbell, E. D. Miller, G. W. Fisher, L. M. Walker, and L. E. Weiss, "Engineered spatial patterns of FGF-2 immobilized on fibrin direct cell organization," Biomaterials, vol. 26, pp. 6762-6770, 2005. 
[86] J. Joseph Thomas Delaney, "REACTIVE INKJET PRINTING AND FUNCTIONAL INKS-A VERSATILE ROUTE TO NEW PROGRAMMED MATERIALS," 2010.

[87] D. L. Baluya, T. J. Garrett, and R. A. Yost, "Automated MALDI matrix deposition method with inkjet printing for imaging mass spectrometry," Analytical chemistry, vol. 79, pp. 6862-6867, 2007.

[88] A. J. Sloane, J. L. Duff, N. L. Wilson, P. S. Gandhi, C. J. Hill, F. G. Hopwood, et $a l .$, "High throughput peptide mass fingerprinting and protein macroarray analysis using chemical printing strategies," Molecular \& Cellular Proteomics, vol. 1, pp. 490-499, 2002.

[89] D. Finnskog, K. Jaras, A. Ressine, J. Malm, G. Marko-Varga, H. Lilja, et al., "High-speed biomarker identification utilizing porous silicon nanovial arrays and MALDI-TOF mass spectrometry," Electrophoresis, vol. 27, pp. 1093-1103, 2006.

[90] D. Ericsson, S. Ekström, J. Nilsson, J. Bergquist, G. Marko-Varga, and T. Laurell, "Downsizing proteolytic digestion and analysis using dispenser-aided sample handling and nanovial matrix-assisted laser/desorption ionization-target arrays," Proteomics, vol. 1, pp. 1072-1081, 2001.

[91] C. A. Parzel, M. E. Pepper, T. Burg, R. E. Groff, and K. J. L. Burg, "EDTA enhances high-throughput two-dimensional bioprinting by inhibiting salt scaling and cell aggregation at the nozzle surface," Journal of tissue engineering and regenerative medicine, vol. 3, pp. 260-268, 2009.

[92] A. Khademhosseini and R. Langer, "Microengineered hydrogels for tissue engineering," Biomaterials, vol. 28, pp. 5087-5092, 2007.

[93] T. Burg, C. A. P. Cass, R. Groff, M. Pepper, and K. J. L. Burg, "Building off-theshelf tissue-engineered composites," Philosophical Transactions of the Royal Society A: Mathematical, Physical and Engineering Sciences, vol. 368, p. 1839, 2010 .

[94] W. Lee, V. Lee, S. Polio, K. Fischer, J. H. Lee, J. K. Park, et al., "Threedimensional cell-hydrogel printer using electromechanical microvalve for tissue engineering," 2009, pp. 2230-2233.

[95] U. Demirci and G. Montesano, "Cell encapsulating droplet vitrification," Lab Chip, vol. 7, pp. 1428-1433, 2007.

[96] M. J. Poellmann, K. L. Barton, S. Mishra, and A. J. W. Johnson, "Patterned Hydrogel Substrates for Cell Culture with Electrohydrodynamic Jet Printing," Macromolecular bioscience, 2011.

[97] E. A. Roth, T. Xu, M. Das, C. Gregory, J. J. Hickman, and T. Boland, "Inkjet printing for high-throughput cell patterning," Biomaterials, vol. 25, pp. 37073715, 2004.

[98] U. Demirci, "Acoustic picoliter droplets for emerging applications in semiconductor industry and biotechnology," Microelectromechanical Systems, Journal of, vol. 15, pp. 957-966, 2006.

[99] U. Demirci and G. Montesano, "Single cell epitaxy by acoustic picolitre droplets," Lab Chip, vol. 7, pp. 1139-1145, 2007. 


\section{VITA}

Riberet Almeida completed his Bachelors in Physics and Computer Applications from Goa University, INDIA in 2003. He completed his MSc in Electronics from the same University in 2005. Thereafter, he graduated with a Masters from the University of Missouri, Columbia in Electrical Engineering in 2008 with a specialization in Nanotechnology. He later completed his $\mathrm{PhD}$ with a specialization in micro / nanoelectromechanical systems (M/NEMS) field at the same university in 2013. 